\title{
Moderator Demonstration Facility Design and Optimization
}

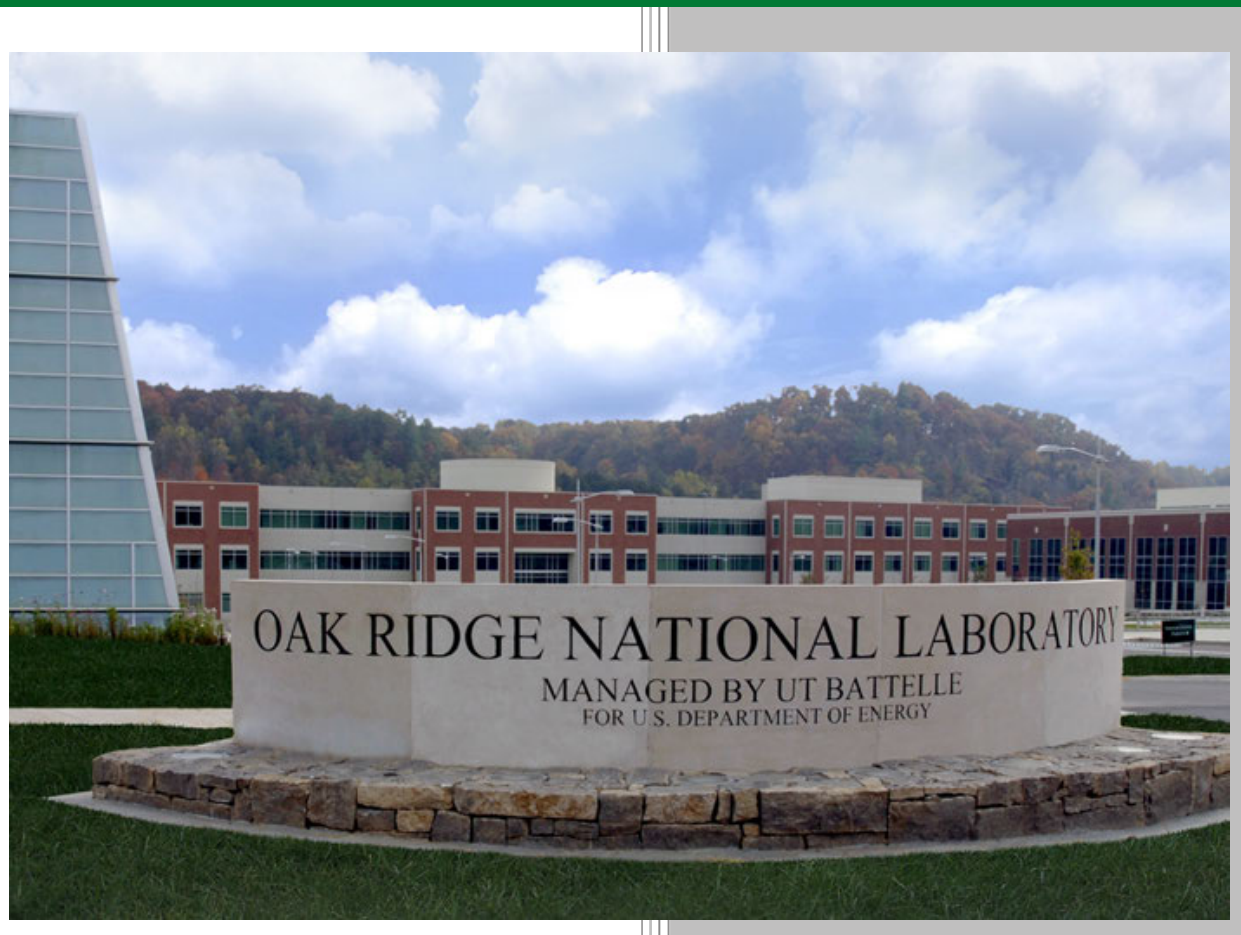

Approved for public release. Distribution is unlimited.
T.C. McClanahan

F.X. Gallmeier

E.B. Iverson

December 2016 


\section{DOCUMENT AVAILABILITY}

Reports produced after January 1, 1996, are generally available free via US Department of Energy (DOE) SciTech Connect.

Website: http://www.osti.gov/scitech/

Reports produced before January 1, 1996, may be purchased by members of the public from the following source:

National Technical Information Service

5285 Port Royal Road

Springfield, VA 22161

Telephone: 703-605-6000 (1-800-553-6847)

TDD: $703-487-4639$

Fax: 703-605-6900

E-mail: info@ntis.fedworld.gov

Website: http://www.ntis.gov/help/ordermethods.aspx

Reports are available to DOE employees, DOE contractors, Energy Technology Data Exchange representatives, and International Nuclear Information System representatives from the following source:

Office of Scientific and Technical Information

PO Box 62

Oak Ridge, TN 37831

Telephone: $865-576-8401$

Fax: 865-576-5728

E-mail: report@osti.gov

Website: http://www.osti.gov/contact.html

This report was prepared as an account of work sponsored by an agency of the United States Government. Neither the United States Government nor any agency thereof, nor any of their employees, makes any warranty, express or implied, or assumes any legal liability or responsibility for the accuracy, completeness, or usefulness of any information, apparatus, product, or process disclosed, or represents that its use would not infringe privately owned rights. Reference herein to any specific commercial product, process, or service by trade name, trademark, manufacturer, or otherwise, does not necessarily constitute or imply its endorsement, recommendation, or favoring by the United States Government or any agency thereof. The views and opinions of authors expressed herein do not necessarily state or reflect those of the United States Government or any agency thereof. 
Instrument and Source Division

\title{
Moderator Demonstration Facility Design and Optimization
}

\author{
T.C. McClanahan \\ F.X. Gallmeier \\ E.B. Iverson
}

Date Published: December 2016

\author{
Prepared by \\ OAK RIDGE NATIONAL LABORATORY \\ Oak Ridge, TN 37831-6283 \\ managed by \\ UT-Battelle, LLC \\ for the \\ US DEPARTMENT OF ENERGY \\ under contract DE-AC05-00OR22725
}




\section{CONTENTS}

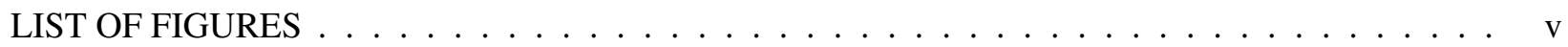

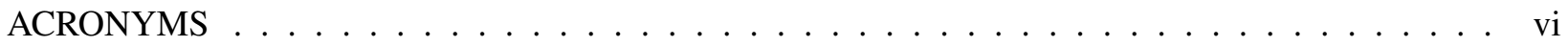

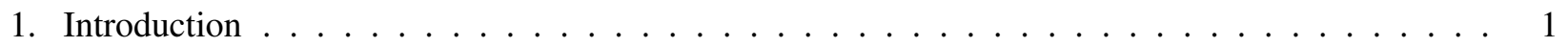

1.1 Background of the Moderator Demonstration Facility . . . . . . . . . . . . . . 1

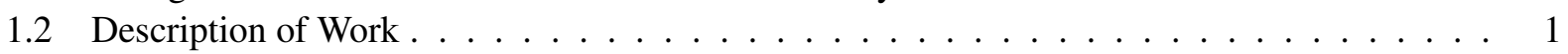

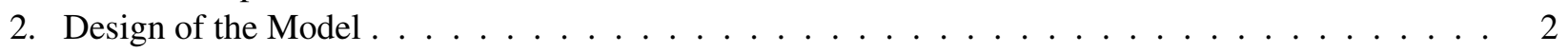

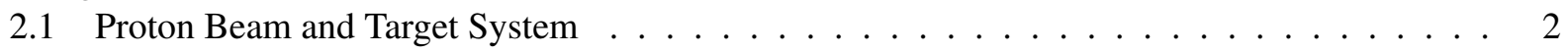

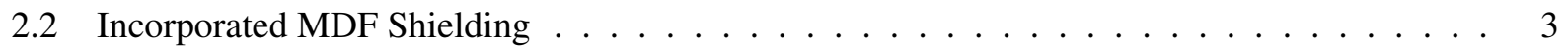

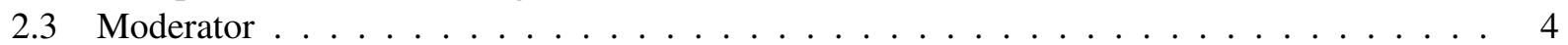

2.4 Neutron Beam . . . . . . . . . . . . . . . . . . . . . . . . 5

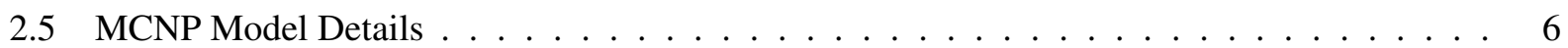

3. Acceleration of Neutron Transport with ADVANTG . . . . . . . . . . . . . 6

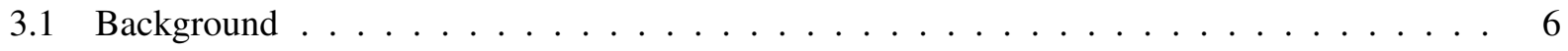

3.2 Choosing the Optimized Tally $\ldots \ldots \ldots \ldots \ldots \ldots$

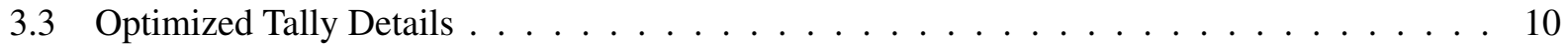

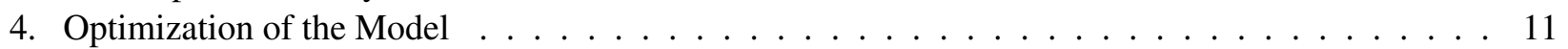

4.1 Optimized Quantities . . . . . . . . . . . . . . . . . . . . 11

4.2 Spatial Distribution Matching . . . . . . . . . . . . . . . . . . . 12

4.2.1 Phase Correlation Image Translation . . . . . . . . . . . . . . . . . . . . . . . . . 12

4.2.2 Chi-Squared Statistic Calculation _ . . . . . . . . . . . . . . . . 14

4.3 Confirmation of the Neutron Emission Time Distribution $\ldots \ldots \ldots$. . . . . . . . 15

4.3 .1 Fitting Routine . . . . . . . . . . . . . . . . . . . 15

4.3.2 FWHM and Beam Intensity Comparisons . . . . . . . . . . . . . . . . 18

4.4 Dakota Results . . . . . . . . . . . . . . . . . . . . 23

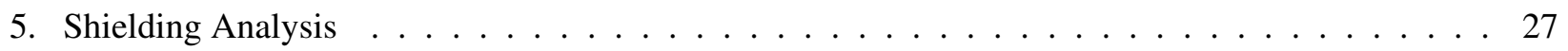

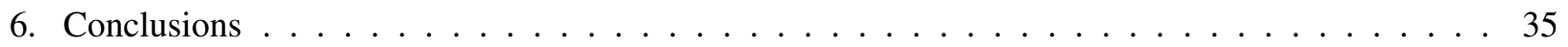

APPENDIX A. Details of Fitting the Ikeda Carpenter Function . . . . . . . . . . . . . A-1

APPENDIX B. Details of Fitting the Beam Intensity $\ldots \ldots \ldots \ldots \ldots \ldots$. . . . . . . . . . 


\section{LIST OF FIGURES}

1 Proton beam spatial elliptical spatial profile given in percentage of the peak. . . . . . . . 2

2 Side view of the MDF target plate and layer of diffusion material. Green is polyethylene, blue is aluminum, red is the diffuser plate . . . . . . . . . . . . 3

3 Water block shielding around MDF target and moderator systems (dark blue - air, light blue - water, green - polyethylene, orange $\left.-\mathrm{B}_{4} \mathrm{C}\right) \ldots \ldots \ldots \ldots$

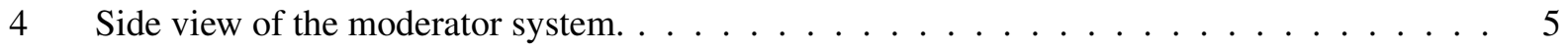

5 View of the neutron beam system (neutron beam coming out of page). . . . . . . . . 5

6 Vertical cross sectional view of MDF with weight window upper-bound parameters for neutrons in the $0.01-10 \mathrm{meV}$ energy bin. . . . . . . . . . . . . . 8

7 Vertical cross sectional view of MDF with weight window parameters for neutrons in the $0.8-1 \mathrm{eV}$ energy bin. . . . . . . . . . . . . . . . . . . 9

8 Vertical cross sectional view of MDF with weight window parameters for neutrons in the $0.9-1.4 \mathrm{MeV}$ energy bin. . . . . . . . . . . . . . . . . . . 9

9 Cell Tally Optimization . . . . . . . . . . . . . . . . . . . . . . . 10

10 Moderator brightness versus emission time for neutrons with energies $5 \pm 0.3 \mathrm{meV}$ for a decoupled water moderator $\ldots \ldots \ldots \ldots \ldots \ldots$

11 Phase Correlation Method . . . . . . . . . . . . . . . . . . . . . 13

12 Comparison of Translation Methods . . . . . . . . . . . . . . . . . . . 14

13 Fitting results of neutron emission time distributions from the MDF $\ldots \ldots \ldots$

14 Fitting results of neutron emission time distributions from the MDF $\ldots \ldots \ldots$

15 Analysis of $10 \mathrm{eV}$ Pulse for Aluminum as the diffusion material . . . . . . . . . . . . . . 19

16 Analysis of $10 \mathrm{eV}$ Pulse for Iron as the diffusion material . . . . . . . . . . . . . . . 20

17 Analysis of $10 \mathrm{eV}$ Pulse for Lead as the diffusion material . . . . . . . . . . . . . . . . . 21

18 Analysis of $10 \mathrm{eV}$ Pulse for Polyethylene as the diffusion material . . . . . . . . . . . 22

19 Reduced $\chi^{2}$ Values for the spatial illumination comparison . . . . . . . . . . . . 23

$20 L^{2}$ Norm Values for the spatial illumination comparison . . . . . . . . . . . . . . . 24

21 Best Match of the spatial illumination between the MDF and STS . . . . . . . . . 25

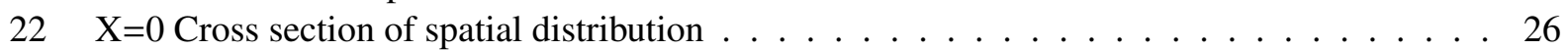

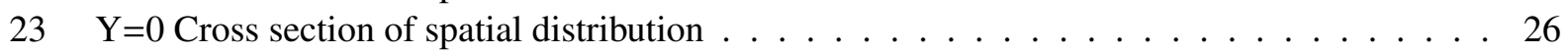

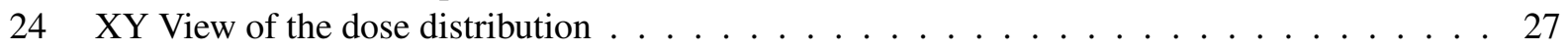

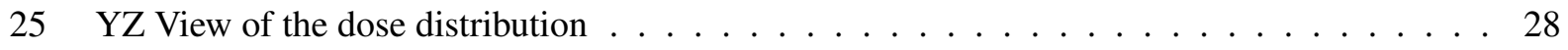

$26 \mathrm{XZ}$ View of the dose distribution . . . . . . . . . . . . . . . . . 28

27 XY View of the dose relative error . . . . . . . . . . . . . . . . . . . . 29

28 YZ View of the dose relative error . . . . . . . . . . . . . . . . . . . . . 29

$29 \mathrm{XZ}$ View of the dose relative error . . . . . . . . . . . . . . . . . 30

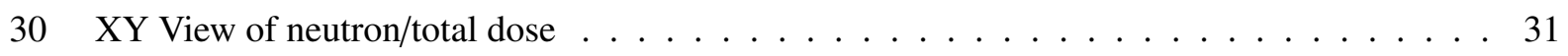

31 YZ View of neutron/total dose . . . . . . . . . . . . . . . . . . . . 32

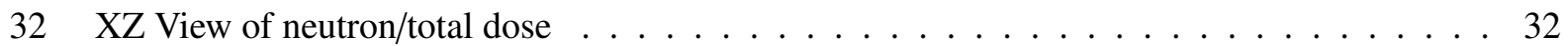

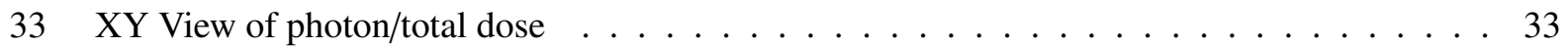

34 YZ View of photon/total dose . . . . . . . . . . . . . . . . . 33

$35 \mathrm{XZ}$ View of photon/total dose . . . . . . . . . . . . . . . . . . . 34

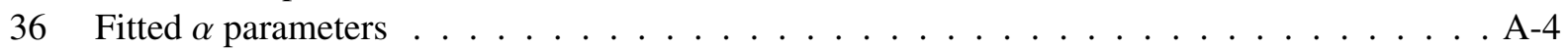

37 Fitted $\Sigma$ parameters $\ldots \ldots \ldots \ldots \ldots \ldots \ldots \ldots \ldots \ldots$

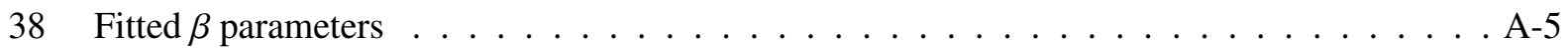


39 Fitted R parameters . . . . . . . . . . . . . . . . . . A A-5

40 Fitted $\mathrm{R}$ and $\beta$ parameters . . . . . . . . . . . . . . . . . . . A 6

$41 S_{1}$ values from the $\alpha$ parameter fit $\ldots \ldots \ldots \ldots \ldots \ldots \ldots$

$42 S_{2}$ values from the $\alpha$ parameter fit $\ldots \ldots \ldots \ldots \ldots \ldots \ldots$

$43 \quad \beta$ values from the $\beta$ paremeter fit . . . . . . . . . . . . . . . . A-9

$44 E_{0}$ values from the R parameter fit $\ldots \ldots \ldots \ldots \ldots \ldots \ldots$

45 Fitted and Simulated MDF Spectral Intensity . . . . . . . . . . . . . B-3

$46 i_{T h} / i_{\text {epi }}$ values from the spectral beam current parameter fit $\ldots \ldots \ldots \ldots \ldots$

$47 \quad E_{T}$ values from the spectral beam current parameter fit $\ldots \ldots \ldots \ldots \ldots$

48 Energy-Integrated Peak Brightness . . . . . . . . . . . . . . . . . . B-5 


\section{ACRONYMS}

ADVANTG Automated Variance Reduction Generator

CADIS Consistant Adjoint Driven Importance Sampling

DAKOTA Design Analysis Kit for Optimization and Terascale Applications

FFT Fast Fourier Transform

FWHM Full Width Half Maximum

IC Ikeda-Carpenter

ITSF Integrated Test Stand Facility

LMFIT Non-Linear Least-Squares Minimization and Curve-fitting

MCNP Monte Carlo N-Particle transport code

MDF Moderator Demonstration Facility

MEBT Medium-energy beam transport

MLE Maximum likelihood estimation

ORNL Oak Ridge National Laboratory

RFQ Radio-frequency quadrapole

RMS Root-Mean-Square

SNS Spallation Neutron Source

STS Second Target Station

TOF Time of Flight 


\section{Introduction}

\subsection{Background of the Moderator Demonstration Facility}

The Spallation Neutron Source (SNS) facility at Oak Ridge National Laboratory (ORNL) is implementing a Moderator Demonstration Facility (MDF) to demonstrate the performance characteristics of advanced moderators central to the Second Target Station (STS) for SNS [1]. The MDF will use the "spare" front-end installation within the SNS accelerator support complex - an ion source, radio-frequency quadrupole (RFQ) accelerator, and medium-energy beam transport (MEBT) chopper - to provide a 2.5 $\mathrm{MeV}$ proton beam of peak current $50 \mathrm{~mA}$ and maximum pulse length of less than $10 \mu \mathrm{s}$ at a repetition rate of no more than $60 \mathrm{~Hz}$ to a suitable neutron-producing target to demonstrate those aspects of moderator performance necessary to meet the goals of the STS design effort. The accelerator beam parameters are not open to variation beyond that described above - they are fixed by the nature of the spare front-end installation (the Integrated Test Stand Facility; ITSF). Accordingly, there are some neutronic challenges in developing prototypic moderator illumination from a very non-prototypic primary neutron source; the spallation source we are attempting to mimic has an extended neutron source volume approximately $40 \mathrm{~cm}$ long (in the direction of the proton beam), approximately $10 \mathrm{~cm}$ wide (horizontally transverse to the proton beam) and approximately $5 \mathrm{~cm}$ high (vertically transverse to the proton beam), and an isotropic evaporation energy spectrum with mean energy above $1 \mathrm{MeV}$. In contrast, the primary neutron source available from the ${ }^{7} \mathrm{Li}(\mathrm{p}, \mathrm{n})$ reaction (the most prolific at $2.5 \mathrm{MeV}$ proton energy by more than an order of magnitude) is strongly anisotropic, with an energy spectrum that is both strongly dependent on emission angle and kinematically limited to less than $700 \mathrm{keV}$, and the interaction zone between the incident protons and any target material (neutron-producing or not) is intrinsically limited to a few tens of microns. The MDF will be unique and innovative amongst the world's moderator test facilities in that it will be intended to be very prototypic in terms of "moderator illumination" - the spatial variation of the neutron flux entering the moderator itself - as well as capable of testing so-called high-brightness moderators in a wing configuration.

\subsection{Description of Work}

During the design phase of the MDF, the Monte Carlo N-Particle transport code MCNPX v.2.7.0 is being used as the primary tool for the neutronic analysis of the facility and characterization of the moderator performance [2]. The Automated Variance Reduction Generator (ADVANTG) code is being utilized to accelerate the MCNPX calculations [3]. The ADVANTG code generates space- and energy-dependent weight window parameters for a successive MCNP run by calculating the adjoint flux with the Denovo discrete ordinates code based on the user defined MCNP tally. We have tested various strategies using ADVANTG to generate weight window parameters to efficiently calculate detailed moderator performance. The Design Analysis Kit for Optimization and Terascale Applications (Dakota) toolkit is being implemented to automate the optimization process of the arrangement of materials having low neutron capture cross sections but varying slowing-down properties below a highly-inclined lithium target and extended proton beam footprint in order to match the variation in neutron flux $\left(\mathrm{n} / \mathrm{cm}^{2} / \mathrm{eV} / \mathrm{s}\right.$ between 0.01 and $10 \mathrm{keV}$ ) on the outer surfaces of both prototypic high-brightness parahydrogen moderators and prototypic conventional parahydrogen moderators, while also providing extremely sharp (in time) neutron pulses [4]. The Dakota toolkit provides a means to execute a parameter study and find which arrangement 
of materials is best suited for matching the illumination of the MDF target/moderator system with the STS target/moderator system. In order to accurately compare the illumination of the STS target/moderator system and the MDF target/moderator system during the Dakota runs, two methods were developed and implemented to quantify how well the spatial distributions of the illumination match and to verify how the arrangement of materials affected the neutron pulse shape coming from a prototypic moderator. Image registration techniques are being used to translate the MDF and STS moderator illumination spatial distributions for comparison using a reduced $\chi^{2}$ value as the figure of merit. In order to verify that the slowing down time is not excessively increased, an intricate fitting routine is being implemented to fit pulse shapes from $0.01 \mathrm{meV}$ to $100 \mathrm{eV}$ to the Ikeda-Carpenter function via the LMFIT Python package [5, 6]. These fits are used to characterize any changes in the neutron pulse shape and verify that the material between the target plate and the moderator does not significantly change the neutron pulse shapes coming from the moderator that is being characterized. A shielding and power limits analysis was also done for the MDF to ensure the facility met radiation safety goals.

\section{Design of the Model}

\subsection{Proton Beam and Target System}

The MDF is being designed to join with an already existing accelerator system utilizing the ITSF. The ITSF will supply a proton beam with an average proton energy of $2.5 \mathrm{MeV}$ and a elliptical spatial distribution with a full width half maximum (FWHM) of $35 \mathrm{~mm}$ in the lower-left to upper-right direction and a FWHM of $31 \mathrm{~mm}$ in the lower-right to upper-left direction. Figure 1 shows a plot of the theoretical proton beam coming from the accelerator on a $80 \times 80 \mathrm{~mm}$ mesh with $1 \mathrm{~mm}$ resolution.

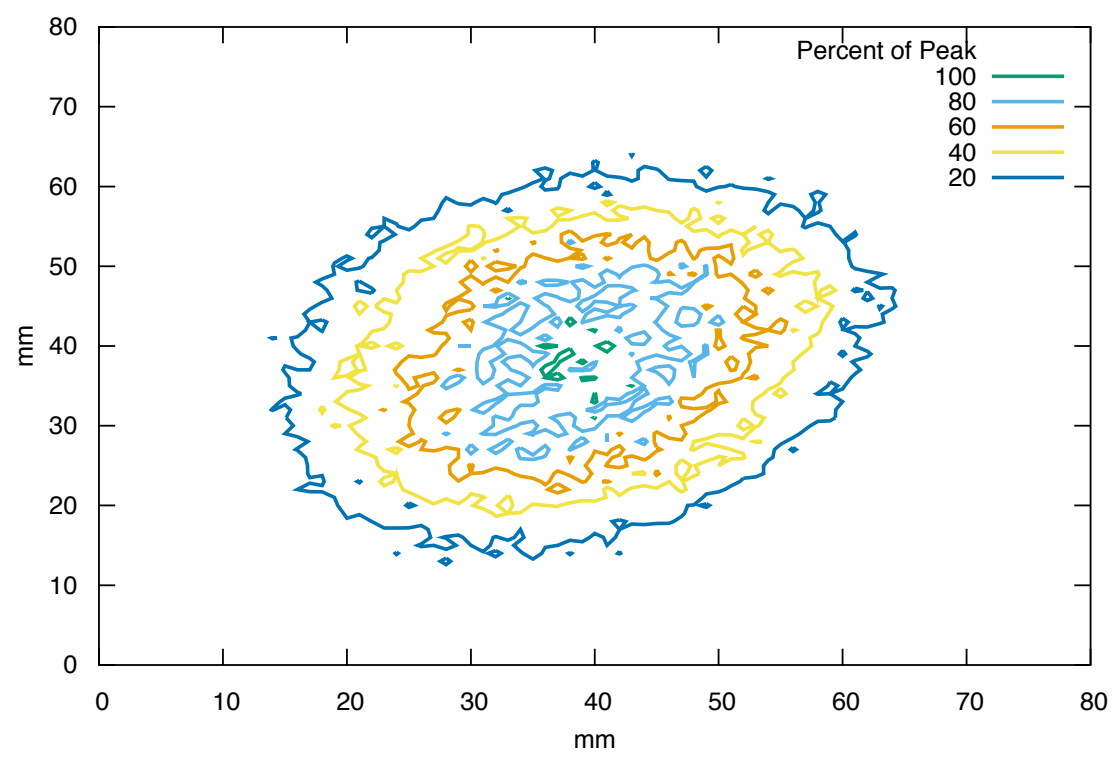

Figure 1. Proton beam spatial elliptical spatial profile given in percentage of the peak.

The proton beam shown in Figure 1 enters the MDF target station at a $27.5^{\circ}$ azimuthal angle and $5^{\circ}$ 
vertical angle from parallel to the floor, and is incident on a lithium coated aluminum plate that is parallel the floor. The lithium coded aluminum plate shown in Figure 2 is a cuboid roughly $8 \times 30 \times 0.635 \mathrm{~cm}$ with the incident proton beam subtending an ellipse with a projected area of approximately $475 \mathrm{~cm}^{2}$. The $\mathrm{Li}^{7}(\mathrm{p}, \mathrm{n}) \mathrm{Be}^{7}$ reaction produces neutrons ranging from 120 and $650 \mathrm{keV}$ in an anisotropic angular distribution that is heavily biased in the forward direction (direction of the incoming proton beam) [7]. As apart of the target system, there is a layer of material beneath the target and above the outermost layer of the neutron beam vacuum vessel (shown in red in Figure 2). This layer of material will act to diffuse the neutrons coming from the MDF target plate in space to match the illumination of the moderators in the STS. The optimization of the combination of materials and the thickness of this layer of material will be a topic of discussion in a later section of this paper.

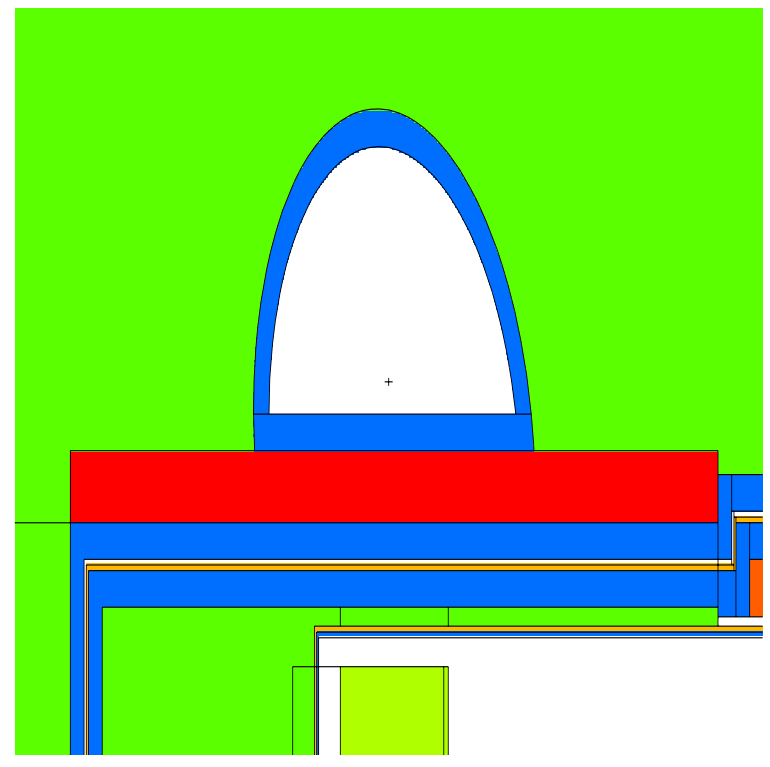

Figure 2. Side view of the MDF target plate and layer of diffusion material. Green is polyethylene, blue is aluminum, red is the diffuser plate

\subsection{Incorporated MDF Shielding}

The major contributer of the MDF's total size consists of various types of shielding materials from interstacked water blocks to layers of polyethylene. Figure 3 shows the water block shielding around the MDF target and moderator in the MCNPX model giving the outside dimensions of $150.9 \times 156.2 \mathrm{x}$ $152.4 \mathrm{~cm}$. The water blocks are each $22.86 \times 15.24 \times 45.72 \mathrm{~cm}$, have $0.635 \mathrm{~cm}$ thick polyethylene walls, and each one has two holes (shown in Figure 3a) where aluminum rods add structural support and allow for several water blocks to be stacked in whatever configuration is desired. Figure $3 \mathrm{~b}$ shows how the water blocks have been stacked for the MDF. The green areas in Figures $3 \mathrm{a} \& 3 \mathrm{~b}$ are customizable blocks of polyethylene that will be made to fit whichever moderator assembly is being studied. The polyethylene acts as both a shielding material and a neutron reflector for the neutrons that leak out of the moderator. Depending on the type of moderator that is being characterized in the MDF, the moderator can be decoupled from the reflection of the neutrons from the polyethylene and the surrounding water blocks by 
the addition of a cadmium liner inside the aluminum vacuum vessel. This cadmium layer acts as a shield against the persisting low-energy neutrons that have reached thermal equilibrium with the reflector material (the polyethylene and water blocks).

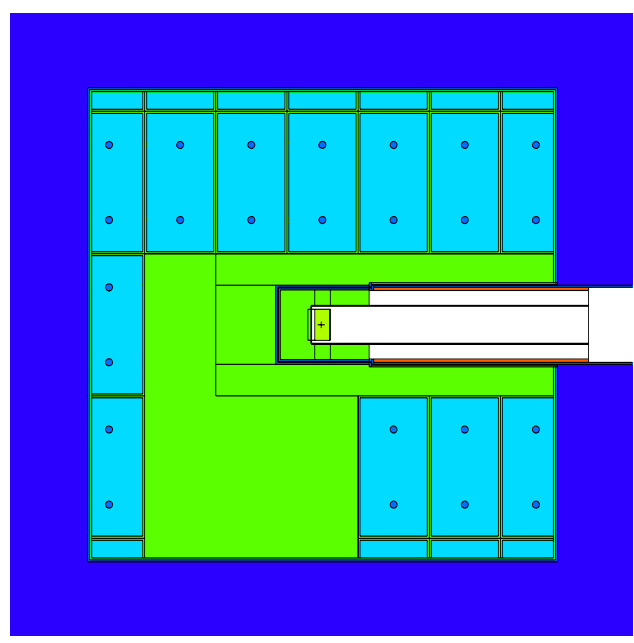

(a) Top view

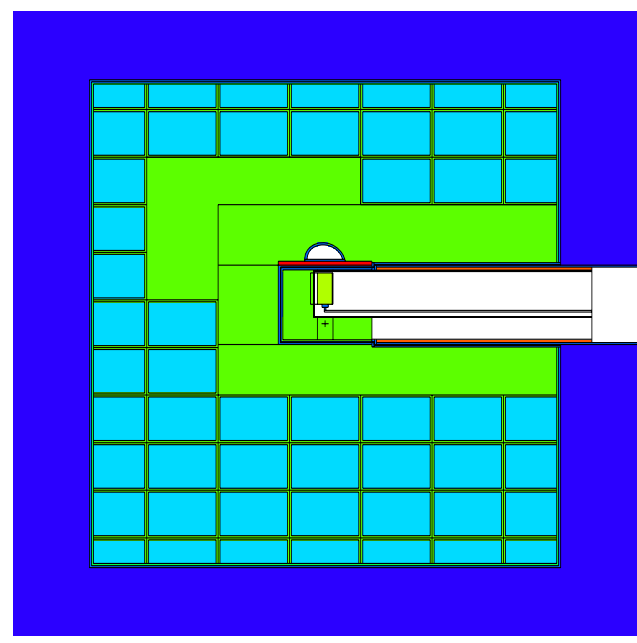

(b) Side view

Figure 3. Water block shielding around MDF target and moderator systems (dark blue - air, light blue - water, green - polyethylene, orange - $\mathbf{B}_{4} \mathbf{C}$ )

\subsection{Moderator}

The moderator system inside the MDF target station is being designed to accommodate a wide variety of moderator shapes and sizes and has the capability to adapt to any number of moderating environments; from decoupled room temperature water to solid methane moderators. In order to adapt to these varying conditions, the whole moderator system is one unit inside a vacuum chamber that can be inserted in and out of the MDF target station on a set of rails. The vacuum vessel is about $1 \mathrm{~cm}$ thick aluminum with an optional $1 \mathrm{~mm}$ thick cadmium decoupler layer inside the vessel. Everything in the vacuum vessel is being designed to mount to the "top" of the vacuum vessel so that by removing the bottom of the vacuum vessel, the user has access to all of the components in the moderator assembly. The ease of access to the system would allow the ability to easily apply a cadmium decoupler, verify the orientation of the moderator, place the thermometry, etc. The moderator will be thermally linked to an outside cryostat via an aluminum arm and will have a heat shield surrounding the moderator. Figure 4 shows a side view of the moderator system with the aluminum thermal link on the bottom of the moderator, the optional cadmium decoupler, and the aluminum heat shield surrounding the moderator. The moderator shown in Figure 4 is a $5 \times 10 \times 10 \mathrm{~cm}$ decoupled water moderator. The green inside of the vacuum vessel in Figure 4 is polyethylene to act as a premoderator, reflector, and shielding. This polyethylene has the potential to be removed or changed to a different material depending on what type of moderator is being characterized. 


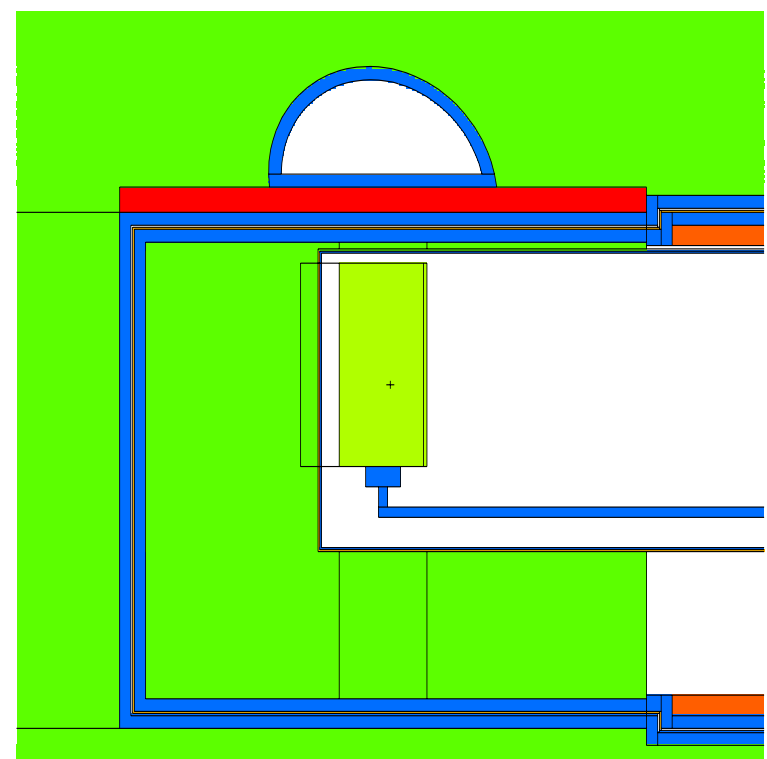

Figure 4. Side view of the moderator system.

\subsection{Neutron Beam}

The neutrons coming from the viewed moderator surface travel down the neutron beam to a suite of detectors and neutron imaging devices. Figure 5 shows the neutron beam system inside and outside the MDF target station.

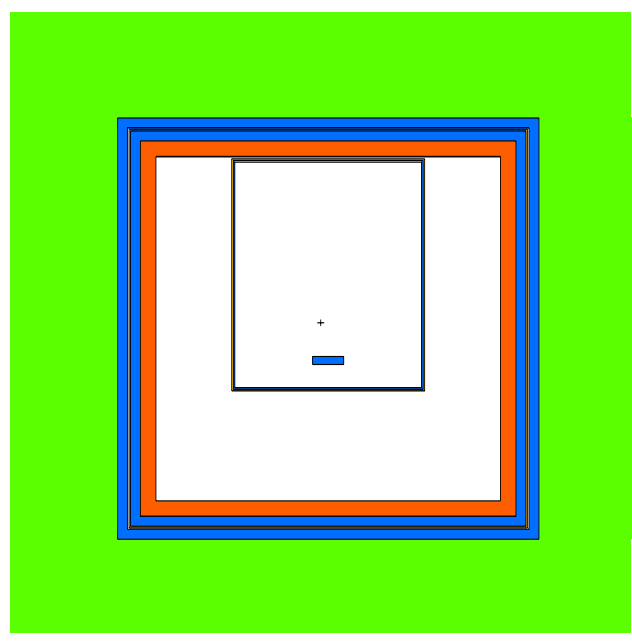

(a) Inside MDF Target Station

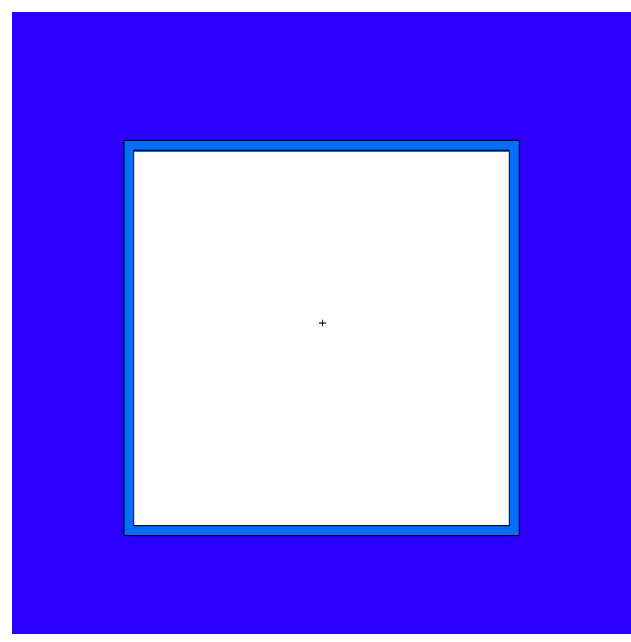

(b) Outside MDF Target Station

Figure 5. View of the neutron beam system (neutron beam coming out of page).

Before the neutrons exit the MDF target station, they travel inside the aluminum vacuum chamber. The portion of the vacuum chamber downstream of the moderator is layered with $1 \mathrm{~cm}$ thick $\mathrm{B}_{4} \mathrm{C}$ (shown in 
orange in Figure 5a) to act as a void liner for the neutron beam. The $\mathrm{B}_{4} \mathrm{C}$ will absorb any neutrons that try to enter the moderator from along the neutron beam line and beyond. To be clear, the neutron beam is not guided along the beamline, it is merely transported in a vacuum to the detector suite at the end of the beamline. The black box in Figure 5a is the heat shield that extends the full length of the neutron beam while inside the MDF target station and the outside green in Figure 5a is a polyethylene block. Figure 5b shows the neutron beam system outside the MDF target station and the system is just the outermost layer of the aluminum vacuum vessel. The darker blue shown in Figure $5 \mathrm{~b}$ is the outside air.

\subsection{MCNP Model Details}

The model described above and shown in the above figures is modeled in MCNP. During the development of the model, the PSTUDY script was used to accelerate the building of the model [8]. In order to calculate the energy, time, and position-dependent brightness of a pulsed neutron source moderator a time-of-flight (TOF) adjusted point detector located $2 \mathrm{~m}$ downstream of the moderator face was used [9]. The details of the TOF adjusted point detector will be discussed in a later section. A mesh tally located in the void space between the diffusion material and the moderator was used to gather the spatial distribution of the neutrons illuminating the moderator. Another mesh tally is placed over the entire geometry to gather the dose information needed for the shielding analysis detailed in a later section of this paper.

\section{Acceleration of Neutron Transport with ADVANTG}

The work in this section was presented at the 2016 ANS Annual Summer Meeting [10].

\subsection{Background}

Calculating the energy, time, and position-dependent brightness of a pulsed neutron source moderator with sufficient detail to optimize its use as a source of slow neutron beams is a challenging process. Modern neutron scattering instrument design methods $[11,12]$ typically require neutron brightness calculations on a moderator (or cold source) surface with transverse spatial resolution of order $1 \mathrm{~cm}$, into a solid angle of less than 0.001 steradian, with energy resolution of no more than $10 \%$ in $\delta E / E$, and with emission time resolution of order $10 \%$ in $\delta t / t[13,14,15,16]$. That is, a single bin in the multidimensional tally space (to use the MCNPX terminology) might cover one square centimeter of moderator emission surface, emitting neutrons into a cone of half angle around one tenth of one degree, between energies of $0.95-1.05 \mathrm{meV}$, at times from 9.5 to 10.5 microseconds following the simulated instantaneous fast neutron pulse. Achieving adequate statistics with such fine coverage is very CPU-intensive, and requires significant variance reduction in order to be practical $[17,18]$. Additionally, optimizing target-moderator-reflector geometries to best match neutron scattering instrument requirements is best done with some level of automated iterative calculation. While such iterative optimization neither needs nor can accommodate such detailed phase space resolution, it also requires significant variance reduction in order to optimize, for example, a figure-of-merit consisting solely of the brightness integrated over the entire $100 \mathrm{~cm}^{2}$ viewed moderator over all emission times for neutron energies below $5 \mathrm{meV}$ [19] via several hundred iterations.

The methods we have developed rely on two primary forms of variance reduction, implemented in MCNPX as weight windows (a system for importance sampling in both physical space and particle energy) and point detectors (a next-event implementation permitting tally contributions to a remote "detector" at 
every scattering collision, not just upon emission from a viewed surface). Using weight windows permits the Monte Carlo simulation to spend significantly more time in more influential portions of the physical system, but requires a fair bit of skill, experience, and manual effort to generate an appropriate set of position and energy dependent weight window parameters in order to succeed. The ADVANTG code system offers the possibility to generate these weight window sets in a more automated fashion than we have previously been able to exploit, offering the chance for significantly improved optimization calculations. The ideal calculation (that is, the one we would most like to optimize) is the above described point detector, placed some meters away from the viewed moderator (or cold source) surface, typically no more than $100 \mathrm{~cm}^{2}$ in area in which the intervening space is a neutron flight path. In order to limit contributions to this point detector tally in the simulation, we typically use the MCNPX "PD" cards to limit contributions to the point detector tally to those cells (physical volumes) we deem relevant to the calculation. This limitation removes the need to model many cubic meters of shielding that would be present in the real system, but do not meaningfully contribute to the point detector, and thus further reduce calculation time spent in noncontributing portions of the system. Additionally, a neutron beam line will in practice incorporate extensive shielding and collimation of high-absorption materials (boron, gadolinium, etc.) to limit the divergence of the neutron beam very precisely, such that only a very specific portion of the viewed moderator (cold source) surface is visible. We simulate this collimation via a zero-importance mask - a void cell lying half-way between the point detector and the viewed moderator surface having half the transverse extent of the desired viewed moderator area with non-zero neutron importance, and a "picture frame" outside that transverse area with zero neutron importance. These variance reduction techniques (point detectors with well-chosen PD cards and zero-importance masks) have been documented to provide multiple order of magnitude increases in calculational efficiency without any loss in accuracy $[15,20]$.

Fig. $3 \mathrm{~b}$ shows the model of the MDF used in the analysis and the location of a point detector $2 \mathrm{~m}$ downstream of the moderator face used to characterize the performance of the moderators. In order to achieve reasonably good statistics on the point detector results, several geometric and tally configurations of the model were analyzed with ADVANTG as well as successive MCNPX runs to determine how to generate the optimum weight windows. The low energy proton-on-lithium neutron source intended for the MDF is strongly anisotropic, with emission energy spectra dependent upon emission angle. We have developed a detailed angle and energy-dependent source term, but found that such a source term cannot be used with ADVANTG, and we were required to use a mono-energetic $1 \mathrm{MeV}$ isotropic point-source instead to generate the weight windows. Choosing the source energy at $1 \mathrm{MeV}$ conservatively extends the energy range beyond the MDF energy range. MCNP models with multiple energy distributions used in the source definition are not supported in ADVANTG. In all of the configurations tested, the Consistent Adjoint Driven Importance Sampling (CADIS) method of generating variance reduction parameters was used to arrive at the weight window parameters.

\subsection{Choosing the Optimized Tally}

Any weight window generation algorithm, including ADVANTG, require a specific tally (more specifically, a single tally) to serve as figure-of-merit in the optimization process. We originally attempted to use the point detector tally to drive this optimization procedure, but found that it worked extremely poorly. A type 4 (volume averaged flux) tally proved much more successful. In the beginning, the point detector tally located $2 \mathrm{~m}$ downstream of the moderator surface with an energy range of 1E-11-20 MeV was used as the 
target tally for ADVANTG to optimize. Fig. 6 shows the weight window lower bound values for the 0.01-10 meV bin with the point detector or the viewed moderator volume used as the optimization tally in ADVANTG.

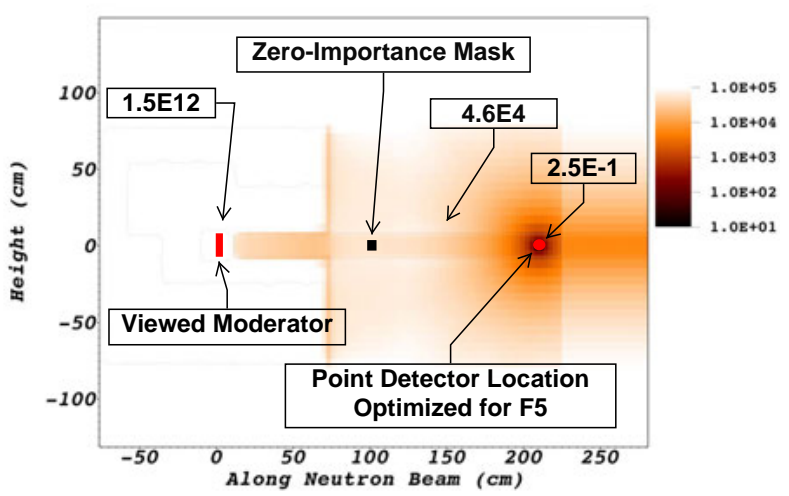

(a) Optimized for Detector Tally

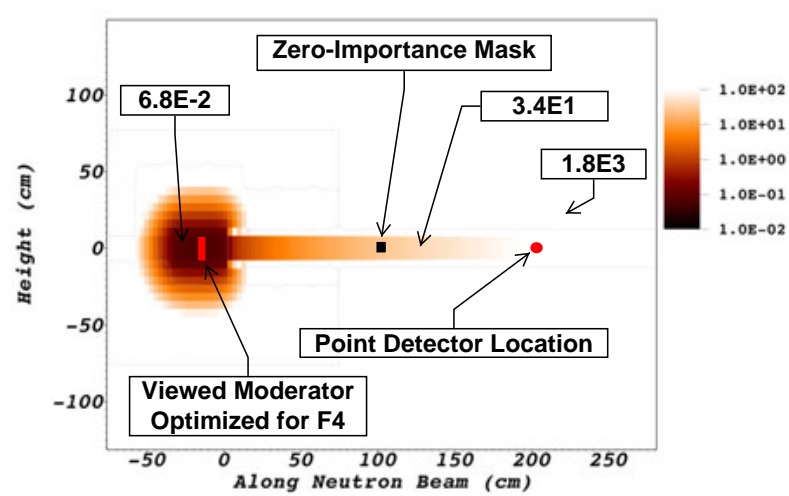

(b) Optimized for Cell Flux Tally

Figure 6. Vertical cross sectional view of MDF with weight window upper-bound parameters for neutrons in the 0.01-10 meV energy bin.

The weight window lower bounds are inversely proportional to the importance. The color scheme in Fig. 6 shows the zones with high weight window parameters (lower importance) with lighter colors and zones with low weight window parameters (higher importance) as darker colors. Fig. 6a shows the weight window lower bounds generated when the point detector was used for the optimization tally and these weight windows resulted in poor statistics in the neutron energy moderator brightness spectra when compared to runs without the weight windows. The results are not only inefficient, they are also incorrect because the weight windows shown in Fig. 6a ignore the zero importance mask. The poor statistics were a result of the source particles being born in lowly weighted areas, where neutrons are subject to Russian Roulette and being killed before they could reach the point detector. A cell flux tally was set to cover the downstream $5 \mathrm{~cm}$ of the viewed moderator volume, again covering the same energy range from 1E-11 MeV-20 MeV. Fig. 6b shows the weight window parameters generated by optimizing based on the cell flux tally. The lower weight window parameters (higher importance area) around the moderating volume in Fig. $6 \mathrm{~b}$ no longer encourage particles to be born in low importance zones and therefore drastically improves the statistics in the neutron energy moderator brightness spectra.

Figs. $7 \& 8$ show the weight window parameters for the $0.8-1 \mathrm{eV}$ and $0.9-1.4 \mathrm{MeV}$ bins respectively. 


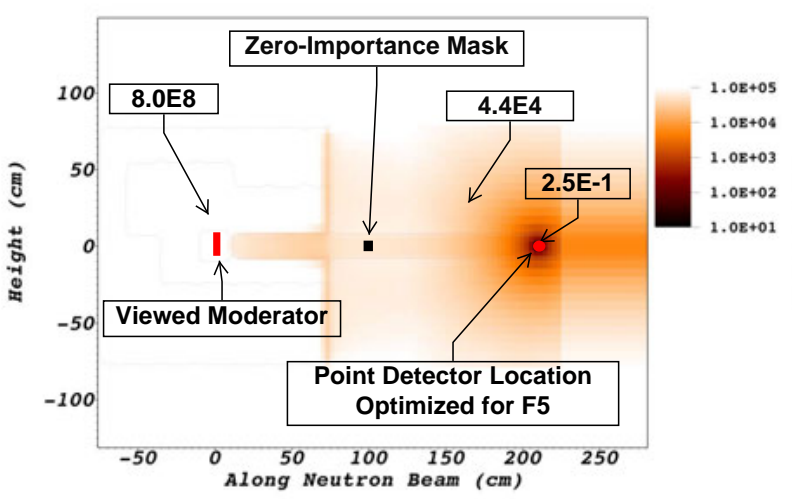

(a) Optimized for Detector Tally

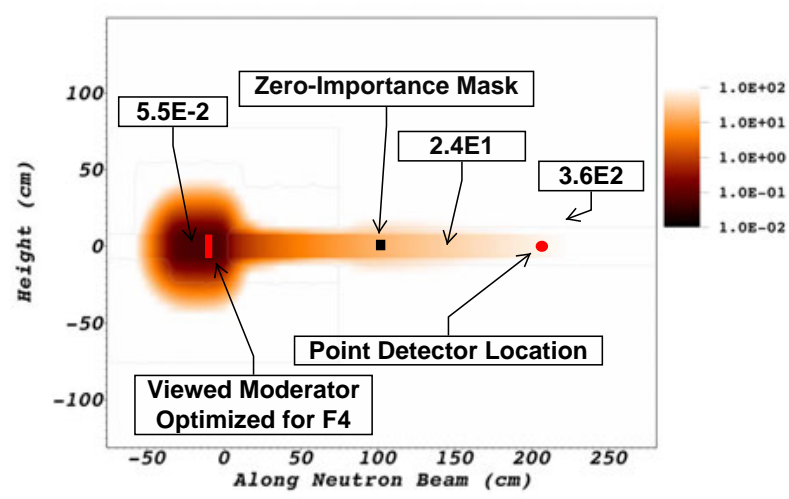

(b) Optimized for Cell Flux Tally

Figure 7. Vertical cross sectional view of MDF with weight window parameters for neutrons in the 0.8-1 eV energy bin.

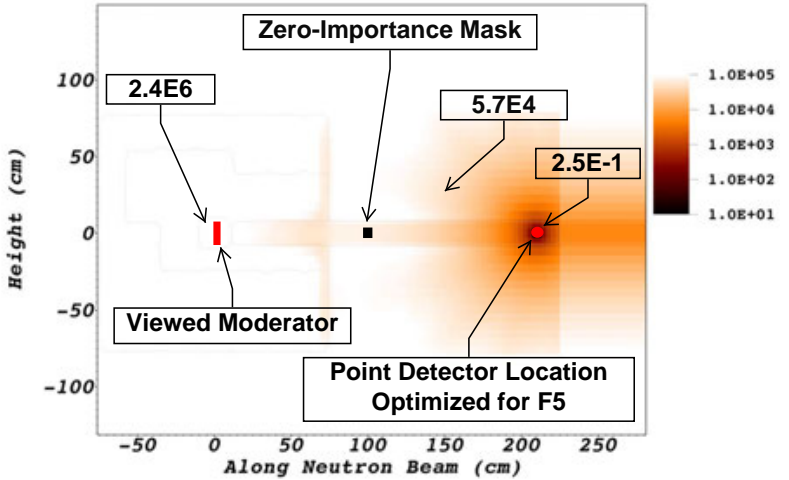

(a) Optimized for Detector Tally

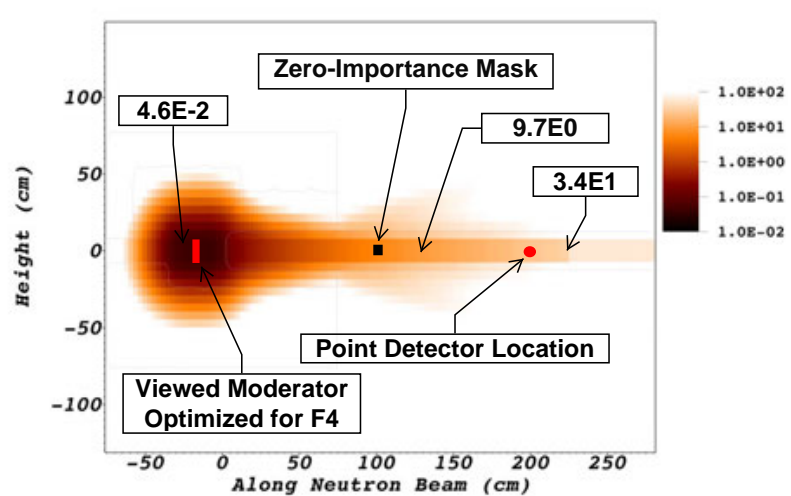

(b) Optimized for Cell Flux Tally

Figure 8. Vertical cross sectional view of MDF with weight window parameters for neutrons in the 0.9-1.4 MeV energy bin. 


\subsection{Optimized Tally Details}

We then optimize the details of the cell flux tally, looking at the energy range and depth of the viewed volume. In order to determine the most effective weight window scheme, the volume encompassed by the cell flux tally and the energy range given to the cell flux tally were varied. For each configuration of the cell flux tally volume and corresponding energy range, ADVANTG was executed to generate the weight windows. Those weight windows were then used in the successive MCNPX run. One thing to note is that the geometry is constant in both the ADVANTG and MCNPX runs in this analysis. Fig. 9 shows the resulting FOM for the energy tally bin surrounding $1 \mathrm{meV}$ of the point detector located $2 \mathrm{~m}$ away from the moderator surface. The FOM shown in Fig. 9 is the inverse of the product of CPU-time and the variance of the tally of interest.

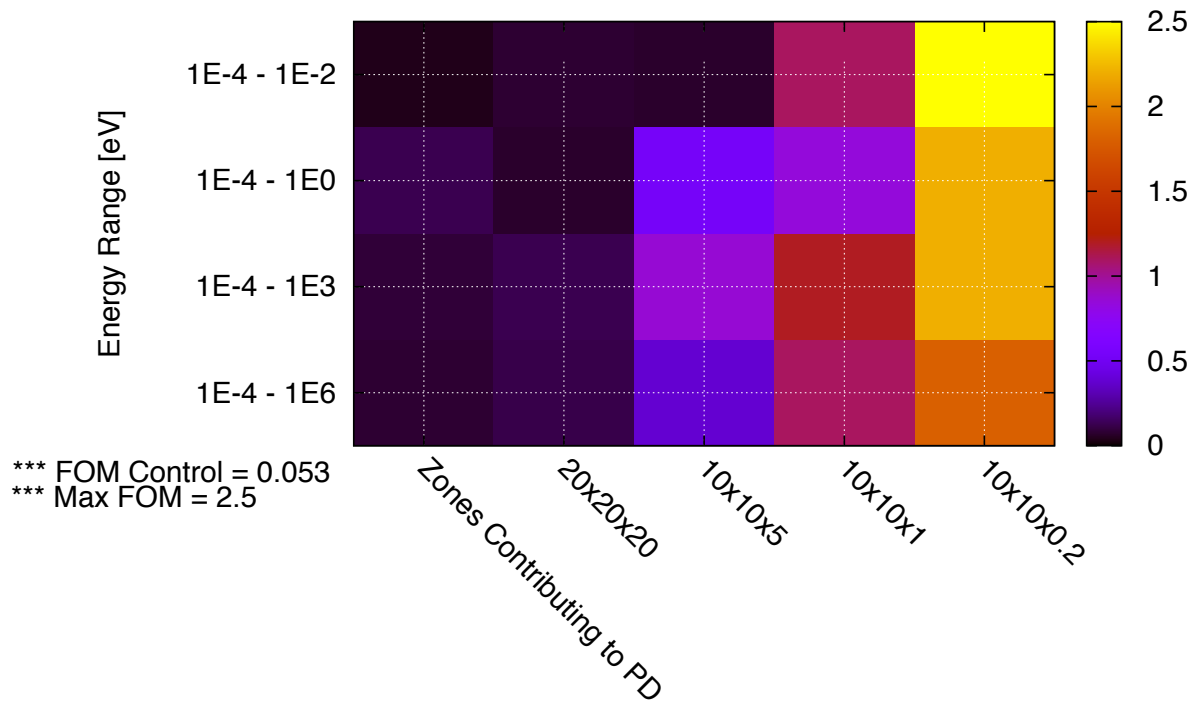

Geometric Volume Coverage $[(\mathrm{cm}) \times(\mathrm{cm}) \times(\mathrm{cm})]$

Figure 9. Cell Tally Optimization

The vertical axis in Fig. 9 represents the energy range given to the cell flux tally and the horizontal axis represents the volume encompassed by the cell flux tally. The Zones Contributing to PD tick on the horizontal axis means that the cell flux tally was given the cells that are on the PD card entry for the point detector, and these cells included the moderator, the polyethylene reflector behind the moderator, the aluminum liner behind the moderator, and the volume of polyethylene reflector located directly behind the $\mathrm{Al}$ liner before the water blocks. The highest FOM was achieved with the cell flux area reduced to $2 \mathrm{~mm}$ thickness in front of the beam-line and with the cell flux energy range restricted to $0.1-10 \mathrm{meV}$. A FOM improvement of almost a factor of 50 was obtained compared against the unbiased control case.

Figure 10 shows the comparison between the neutron brightness spectra for a decoupled water moderator 
with and without the use of ADVANTG generated weight windows with each calculation having the same computational time: $500 \mathrm{CPU}$ minutes, which provided 1E7 histories without the ADVANTG generated weight windows, and 1.2E8 histories with them. The ADVANTG weight windows were generated using the $10 \times 10 \times 0.2 \mathrm{~cm}^{3}$ cell tally volume and the $0.1 \mathrm{meV}-10 \mathrm{meV}$ energy range. Figure 10 illustrates the benefit of the optimally generated weight windows in neutron brightness calculations.

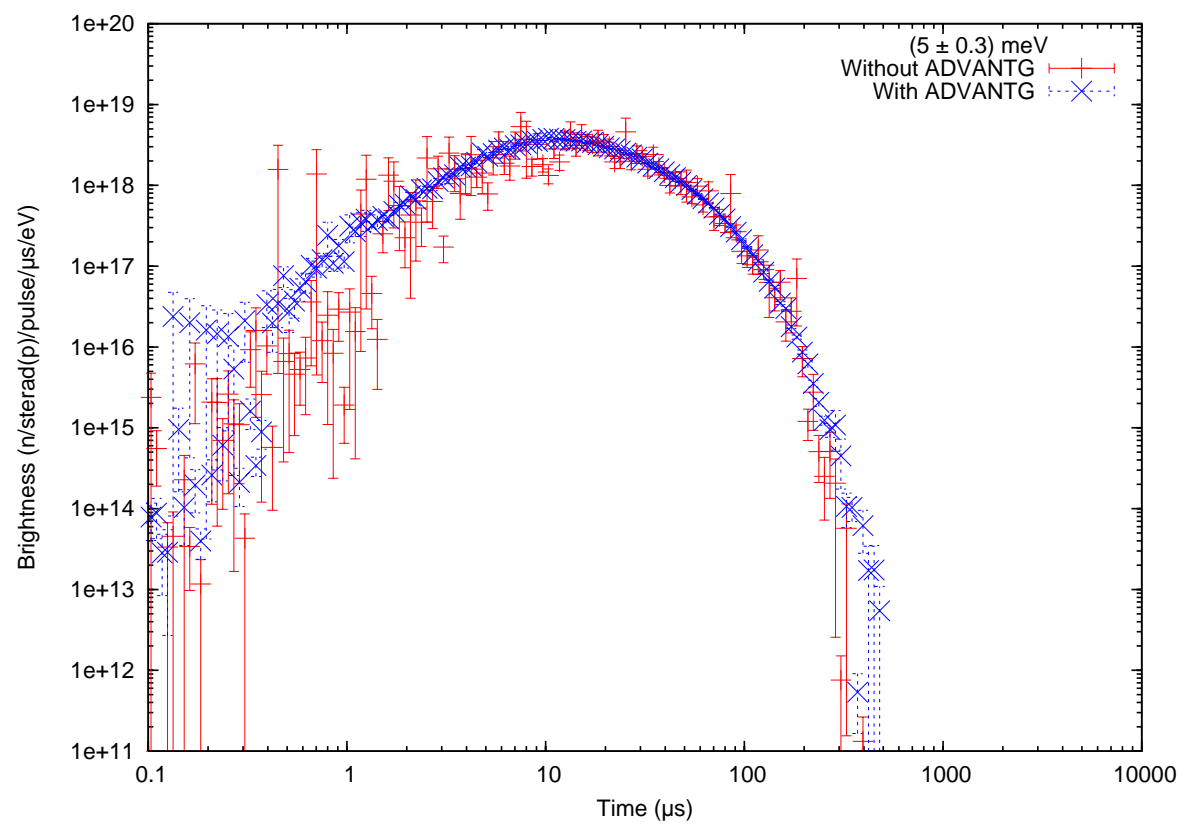

Figure 10. Moderator brightness versus emission time for neutrons with energies $5 \pm 0.3 \mathrm{meV}$ for a decoupled water moderator

\section{Optimization of the Model}

\subsection{Optimized Quantities}

One of the main goals of the MDF is to facilitate an environment surrounding the moderator that is as similar to the STS as one can achieve. In this paper, we specifically examine how changing the composition and thickness of a "diffuser plate," material lying between the neutron producing target and the moderator itself, can make the spatial illumination of the moderator best match that for a spallation source. The spatial illumination of the moderator is the time, energy, and directional dependent flux entering the moderator at any point on the moderating surfaces. While trying to match the spatial distribution of the neutrons entering the moderator, we also had to confirm that the neutron emission time distribution of the moderator is not significantly altered by the optimization of the spatial illumination of the moderator. During the optimization, Dakota's parameter study tool is used to find the optimum configuration of the diffusion 
material based on the spatial distribution matching and the characteristic moderator parameters [4].

\subsection{Spatial Distribution Matching}

We find that image registration techniques using the phase correlation provide the quickest and most effective way to find the best position of the moderator below the source in order to best match the fast neutron flux illumination in the MDF to a comparable illumination on a spallation source.

\subsubsection{Phase Correlation Image Translation}

Image registration techniques have long been used in image processing to compare two or more images [21]. The phase correlation method is an image registration technique used to determine the relative translative, scale, and rotational shift between two similar images by using the fast Fourier transforms (FFTs) of the images [22]. The FFTs of the images are used to produce a cross-power spectrum, and the inverse FFT of the cross-power spectrum results in a cross correlation matrix with a distinct peak corresponding to the relative translative shift between the two images [21,23]. The algorithm used in the optimization for finding the relative translative shift is as follows:

1. Compute the FFT of both images, where $F(u, v)$ is the FFT of the MDF spatial distribution and $G(u, v)$ is the FFT of the STS spatial distribution.

2. Compute the cross-power spectrum, $F(u, v) G^{*}(u, v)$ where $G^{*}(u, v)$ is the conjugate of $G(u, v)$

3. Compute the inverse FFT of the cross-power spectrum to produce the cross correlation matrix

4. Find the location of the maximum in the cross correlation matrix (the location corresponds to the relative shift between the two images)

Figure 11 shows the two input images (Figures 11a \& 11b), the computed cross correlation (Figure 11c), and the resulting translated image (Figure 11d). Figure 11a shows the spatial distribution of neutron flux with energies from $1 \mathrm{eV}$ to $1 \mathrm{keV}$ without the diffuser plate. The mesh tally used to produce Figure 11a is placed in the void space between the inner aluminum wall of the MDF target station and the outside decoupler layer of the moderator vacuum vessel. 


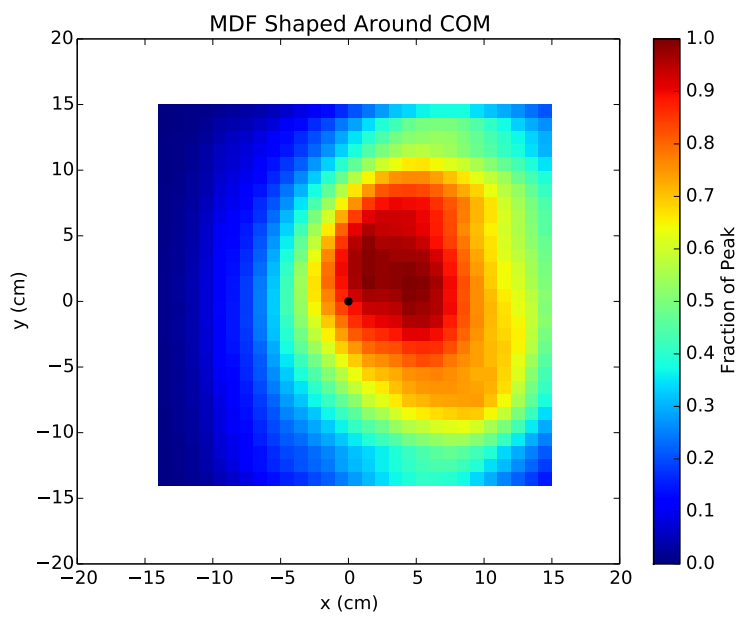

(a) MDF Spatial Distribution without diffuser plate, $F(u, v)$

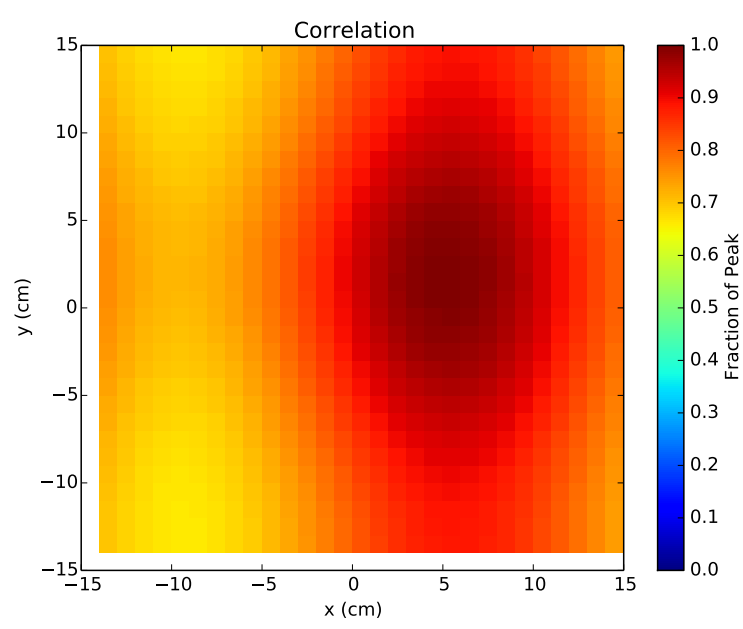

(c) Cross Correlation, $\mathbf{F}(\mathbf{u}, \mathbf{v}) \mathbf{G}^{*}(\mathbf{u}, \mathbf{v})$

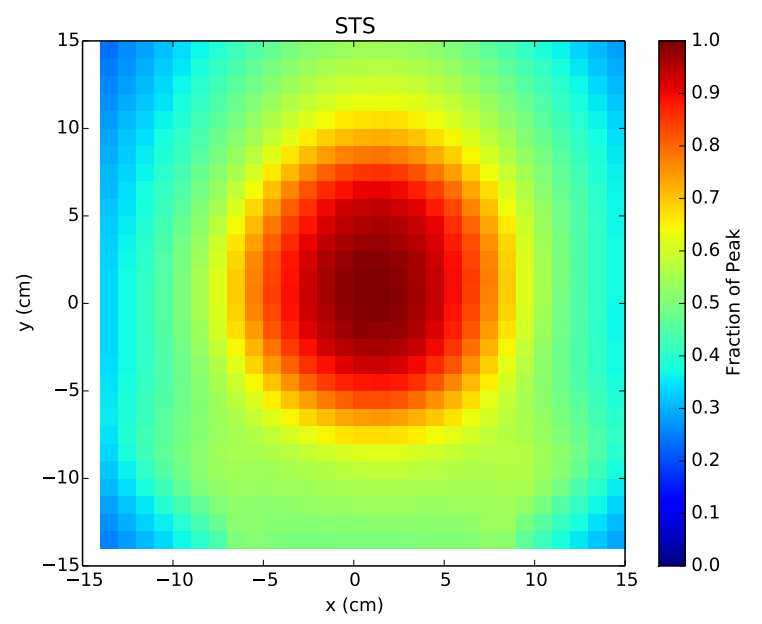

(b) STS Spatial Distribution, G(u,v)

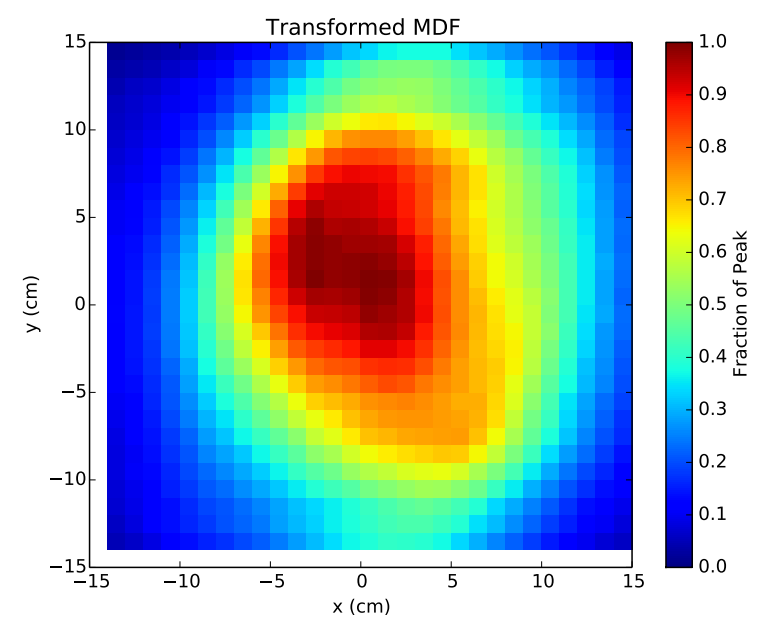

(d) Translated MDF Spatial Distribution

Figure 11. Phase Correlation Method 
The above phase correlation method proves to be the most accurate estimate of the relative translation between two similar images when compared to other methods. The other methods used in the comparison are as follows:

- Shifting the image by the difference in the location of the peaks in the two images

- Shifting the image by the difference in the location of the center of mass (COM) of the two images

- Shifting the image by a series of $\Delta Y \mathrm{~s}$ ("brute force method") and calculate a $\chi^{2}$ value for each shift

Figure 12 shows the comparison in the translative methods. The figure of merit is the reduced chi-squared of the translated MDF data and the STS data and the translation corresponding to the lowest reduced chi-squared is best.

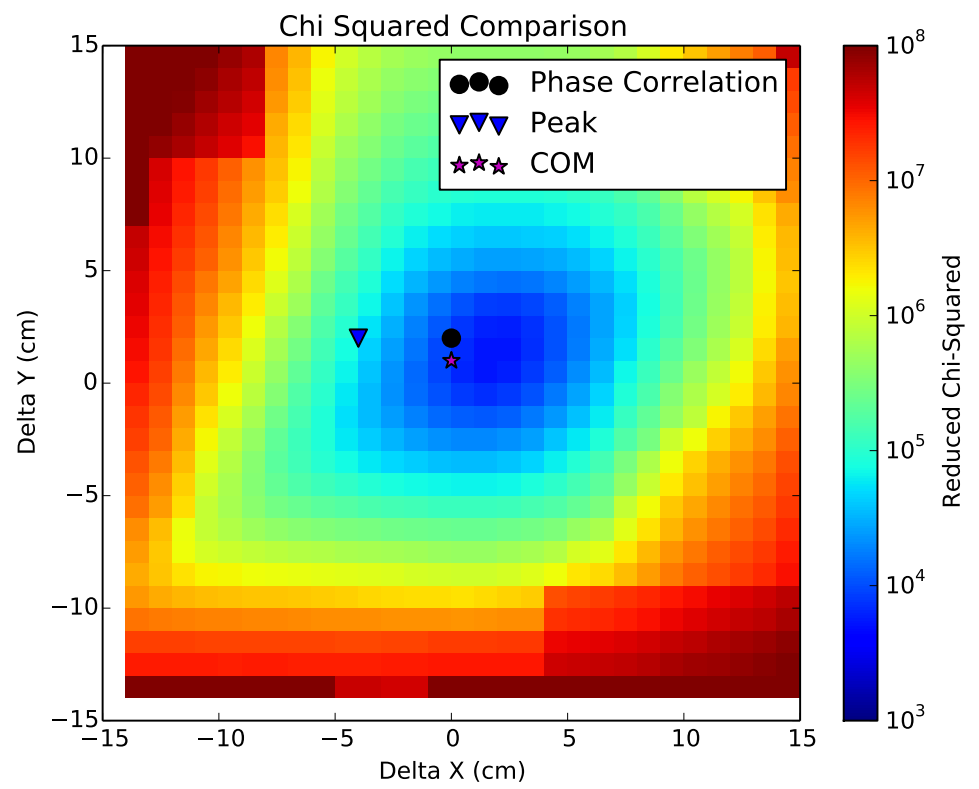

Figure 12. Comparison of Translation Methods

\subsubsection{Chi-Squared Statistic Calculation}

Once the spatial distributions of the MDF and STS have been aligned, a chi-squared statistic is calculated for the goodness-of-fit estimation of the two spatial distributions. The chi-squared statistic that is calculated in this optimization routine is shown in Equation 1:

$$
\chi^{2}=\sum_{i} \frac{\left(F(x, y)_{i}-G(x, y)_{i}\right)^{2}}{\sigma_{F(x, y)_{i}}^{2}+\sigma_{G(x, y)_{i}}^{2}}
$$

where $\mathrm{F}(\mathrm{x}, \mathrm{y})$ is the MDF spatial distribution, $\mathrm{G}(\mathrm{x}, \mathrm{y})$ is the STS spatial distribution, and $\sigma$ is the standard deviation of the spatial distributions. 


\subsection{Confirmation of the Neutron Emission Time Distribution}

While trying to best match the MDF spatial distribution with the STS spatial distribution, we have to ensure that the neutron emission time distribution of the moderator will not be altered.

\subsubsection{Fitting Routine}

A Python script was developed utilizing the Non-Linear Least-Squares Minimization and Curve-Fitting for Python (LMFIT) package to fit both the Ikeda-Carpenter (IC) and spectral beam current function [6, 5]. LMFIT is built upon the Scipy Python Library and offers a powerful suite of tools for data fitting [24]. The user gives LMFIT a dataset to fit to a user-defined model, defines a set of parameters within the model by allocating parameter values, ranges, whether LMFIT is allowed to vary the parameter, etc., and then LMFIT applies an optimization/minimization routine to find which parameter set gives the minimal chi-squared value between the original dataset and the model. After the optimal parameter set is found, LMFIT provides many tools to display the outcome of the fitting routine. The IC function is a physically inspired formulation of the emission time distribution of a pulsed-source moderator [5].

The fitting routine described above was used to fit the neutron emission time distributions to the IC and spectral beam current functions. The details of these fits are described in APPENDIX A. and APPENDIX B. Figures 13 \& 14 shows the neutron emission time distributions for neutron energies of 1 , 10,100 , and $1000 \mathrm{meV}$, and the three fits corresponding to the three weighting schemes used in the fitting routine for the case of $5 \mathrm{~cm}$ of aluminum between the moderator and the source. 


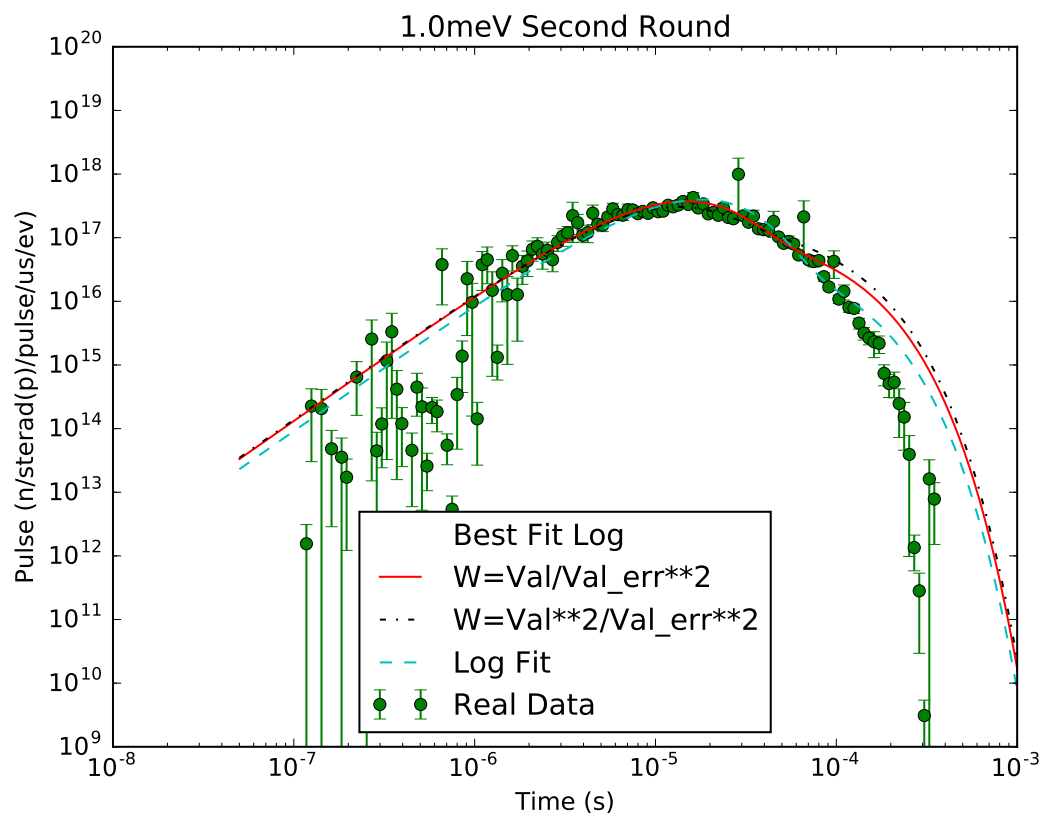

(a) $1 \mathrm{meV}$

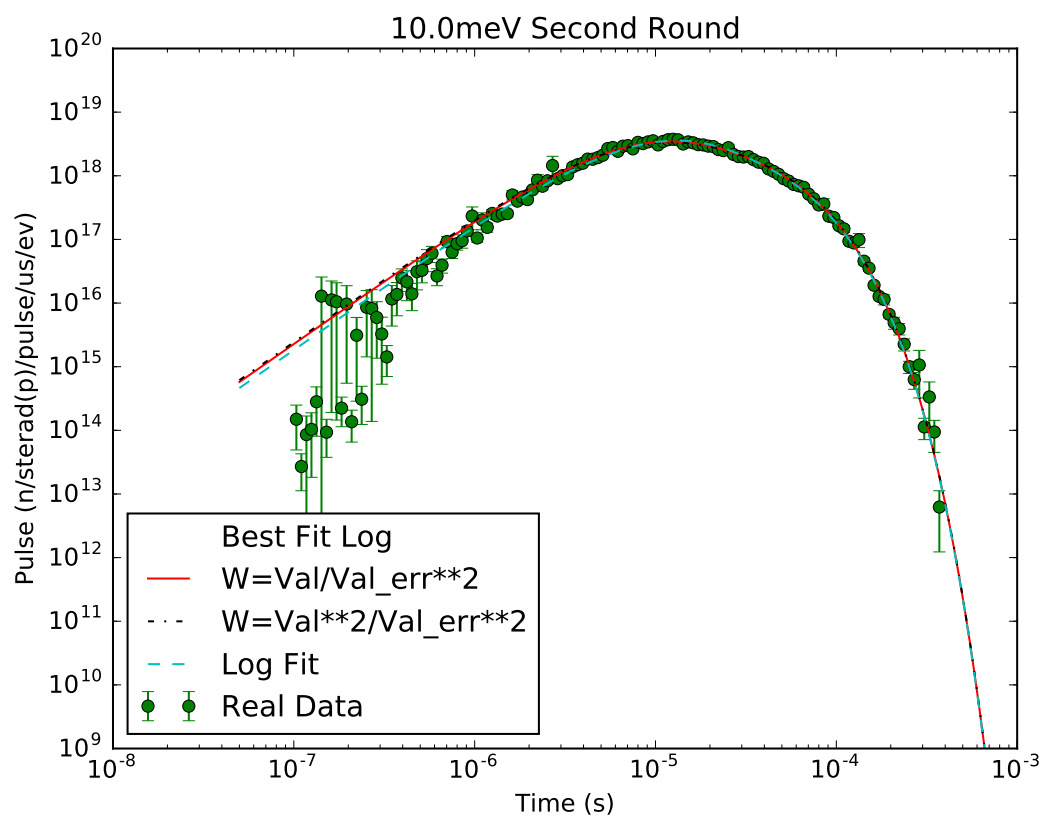

(b) $10 \mathrm{meV}$

Figure 13. Fitting results of neutron emission time distributions from the MDF 


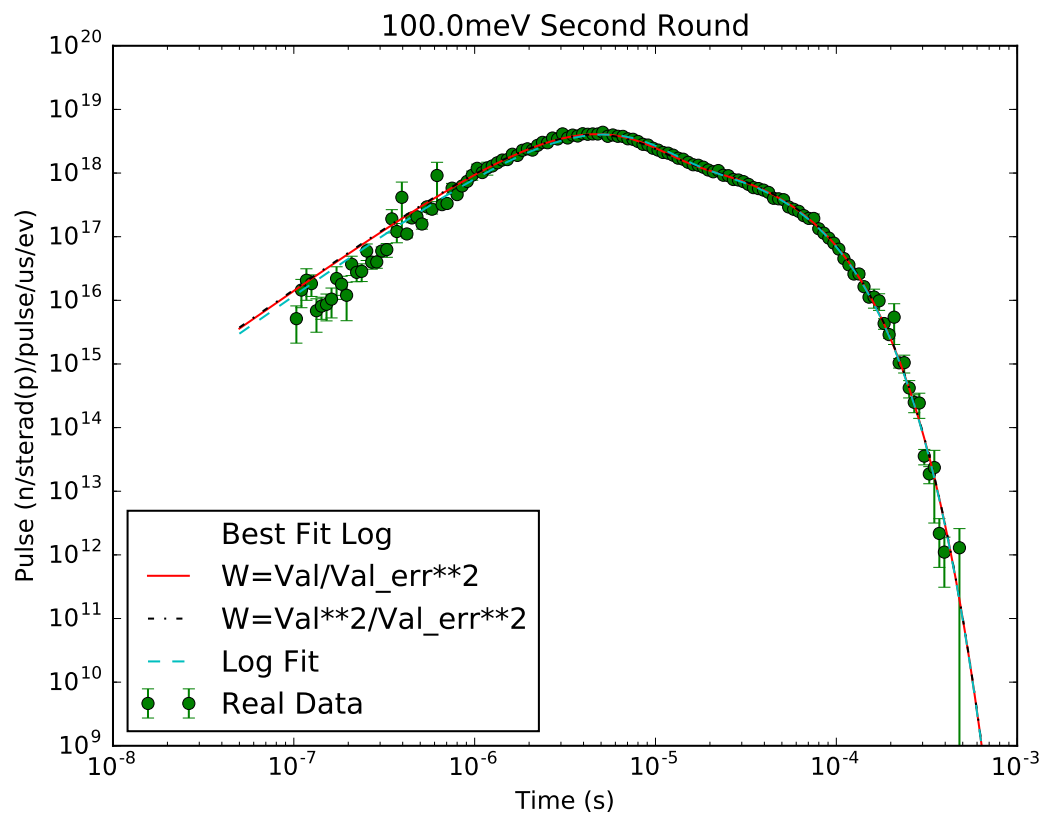

(a) $100 \mathrm{meV}$

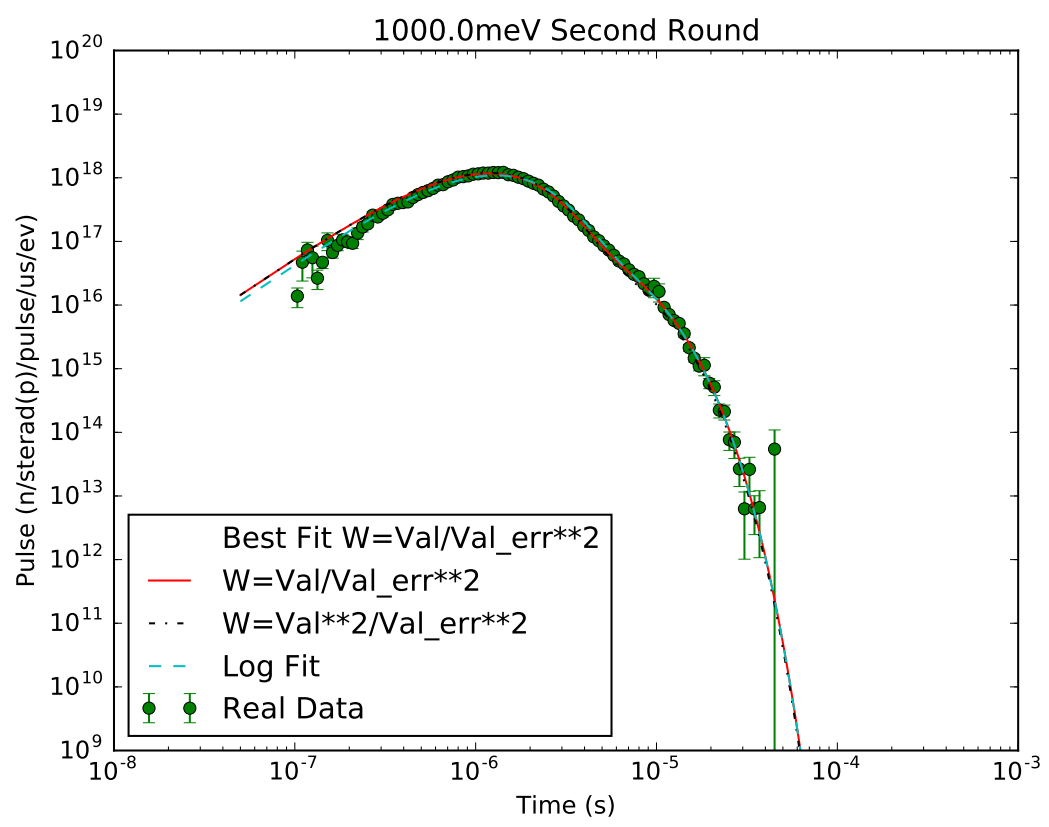

(b) $1 \mathrm{eV}$

Figure 14. Fitting results of neutron emission time distributions from the MDF 


\subsubsection{FWHM and Beam Intensity Comparisons}

Since the analytical pulse shape fit did not do particularly well, constancy of the neutron emission time distribution had to be verified via other techniques. The FWHM and time-integrated value (Beam Current) of the neutron emission time distributions at $10 \mathrm{eV}$ were calculated and the resulting values are shown in Figures 15a, 16a, 17a, \& 18a. Figures 15a, 16a, 17a, \& 18a show that the FWHM does not deviate more than $10 \mu$ s from the original value and that the beam current does not drop more than a factor of 6 for any of the diffusion materials at any thicknesses evaluated in this optimization study. Figures $15 \mathrm{~b}, 16 \mathrm{~b}, 17 \mathrm{~b}, \&$ $18 \mathrm{~b}$ shows the $10 \mathrm{eV}$ pulse shapes for each of the materials in the study at various thicknesses. These figures show that there is no significant variation of the neutron emission time distributions for the various materials and will allow the best material chosen by the Dakota optimization routine to be directly implemented in the MDF. 


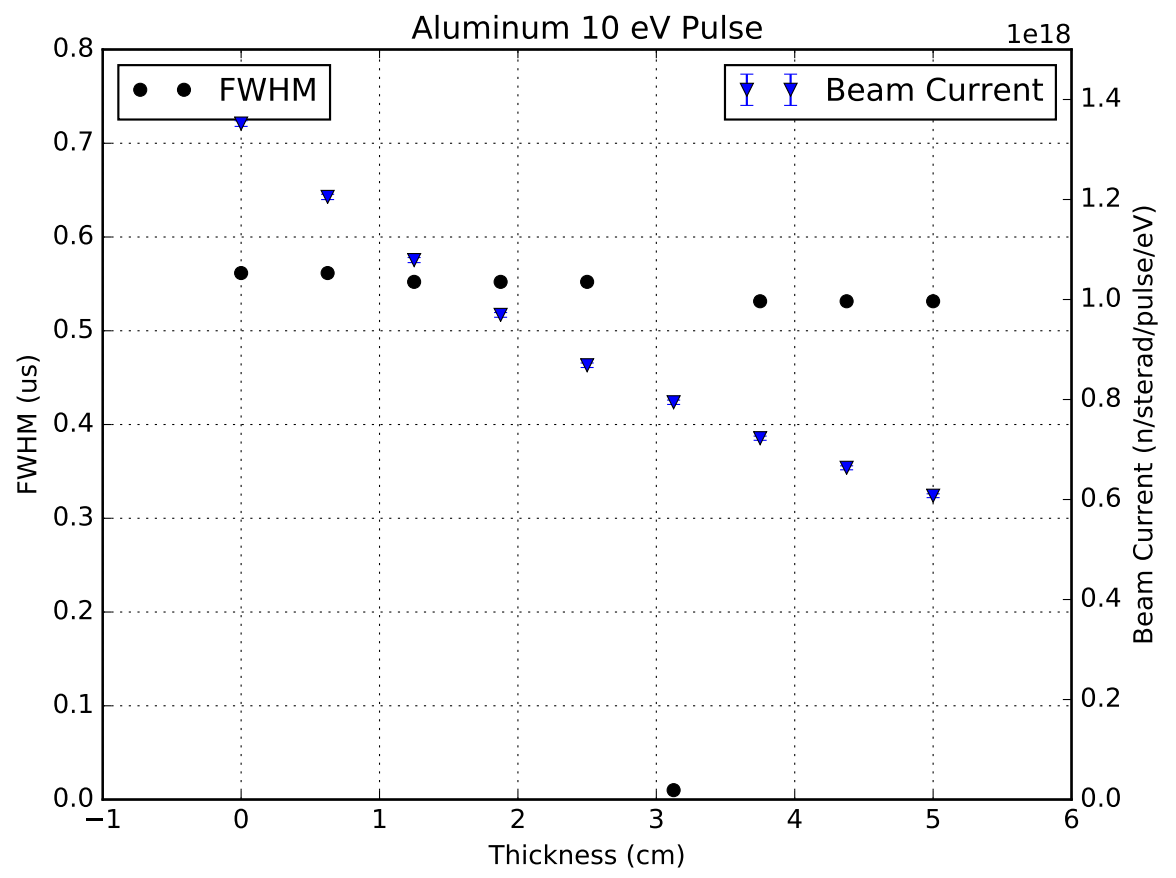

(a) FWHM and Beam Intensity

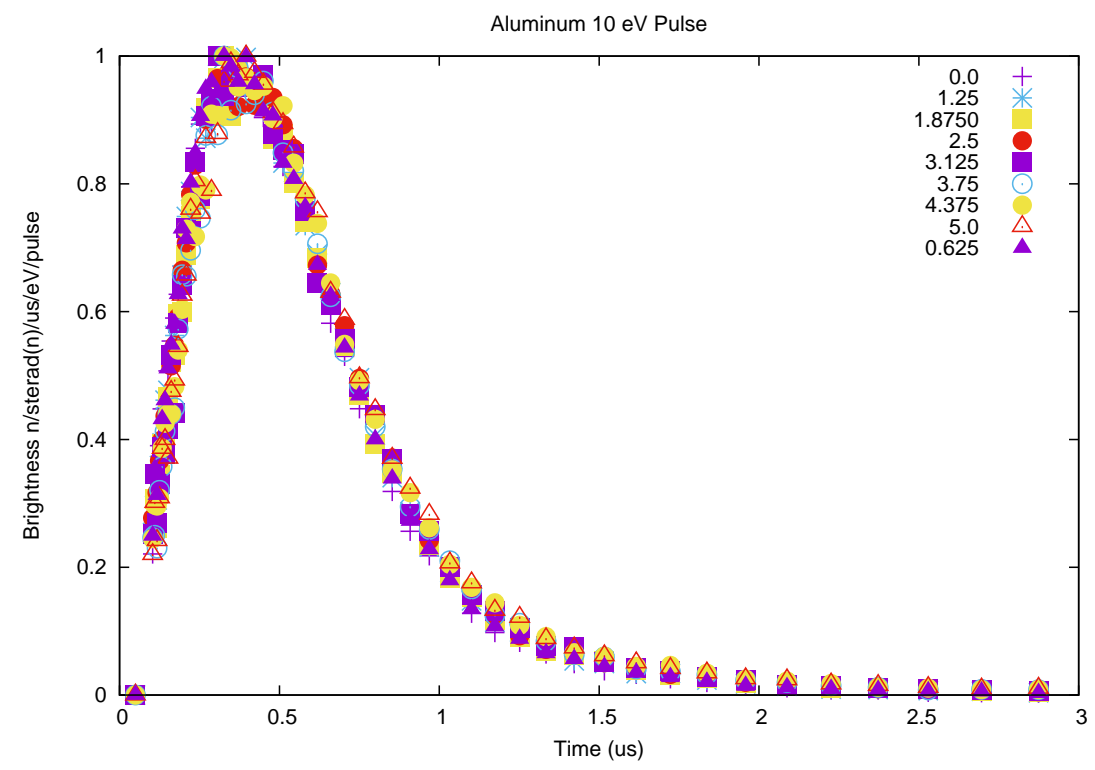

(b) Neutron Pulse Shapes

Figure 15. Analysis of $10 \mathrm{eV}$ Pulse for Aluminum as the diffusion material 


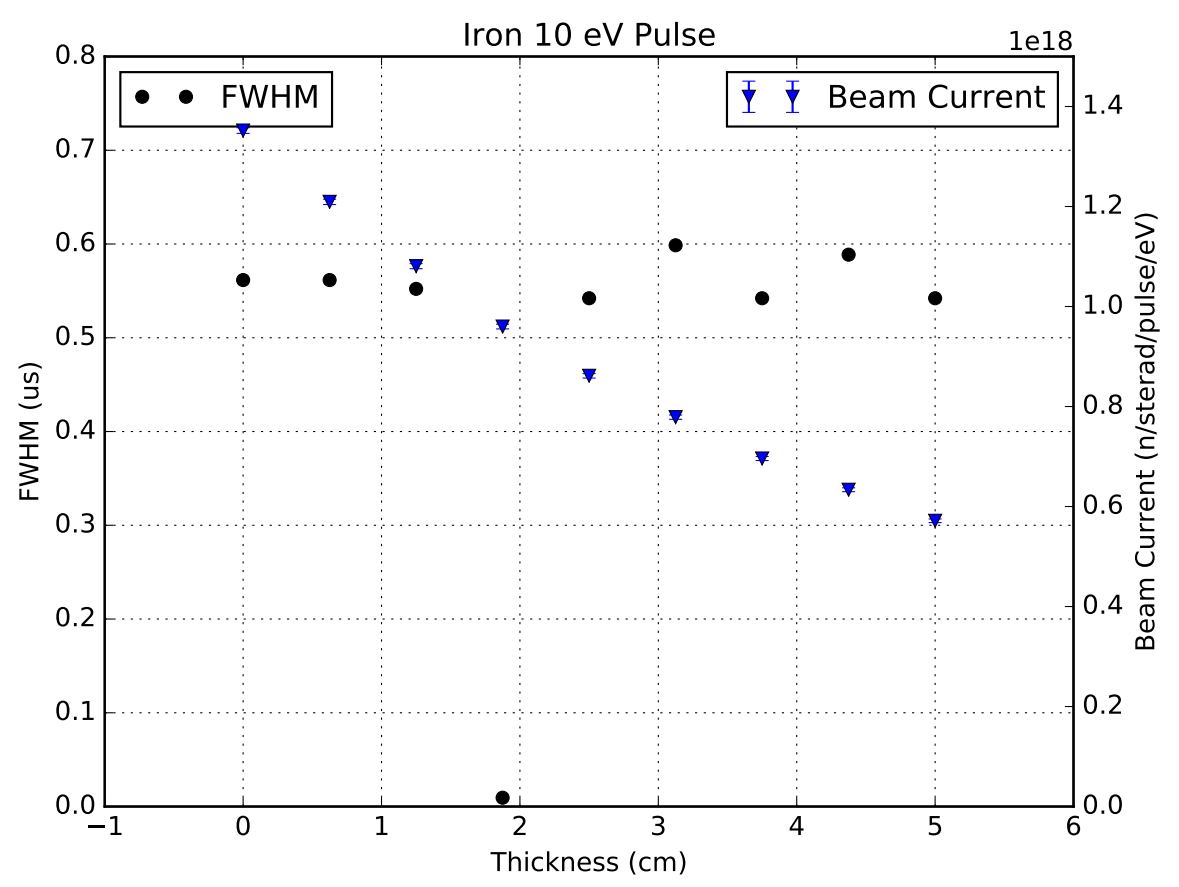

(a) FWHM and Beam Intensity

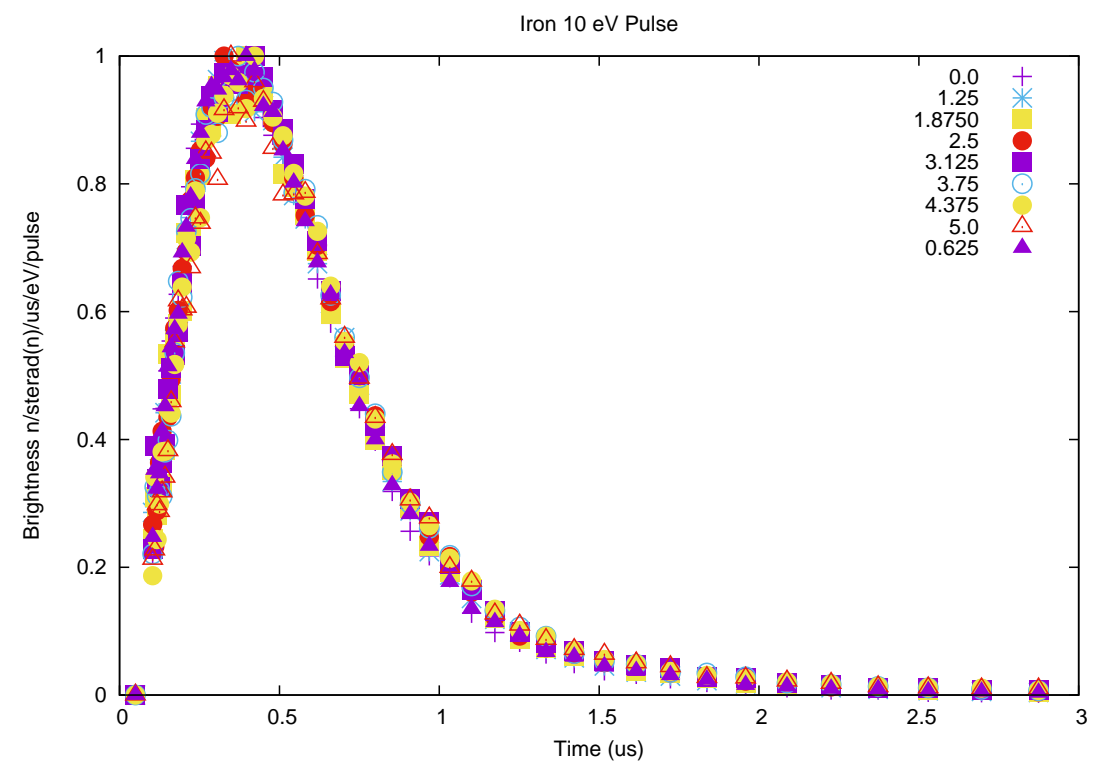

(b) Neutron Pulse Shapes

Figure 16. Analysis of $10 \mathrm{eV}$ Pulse for Iron as the diffusion material 


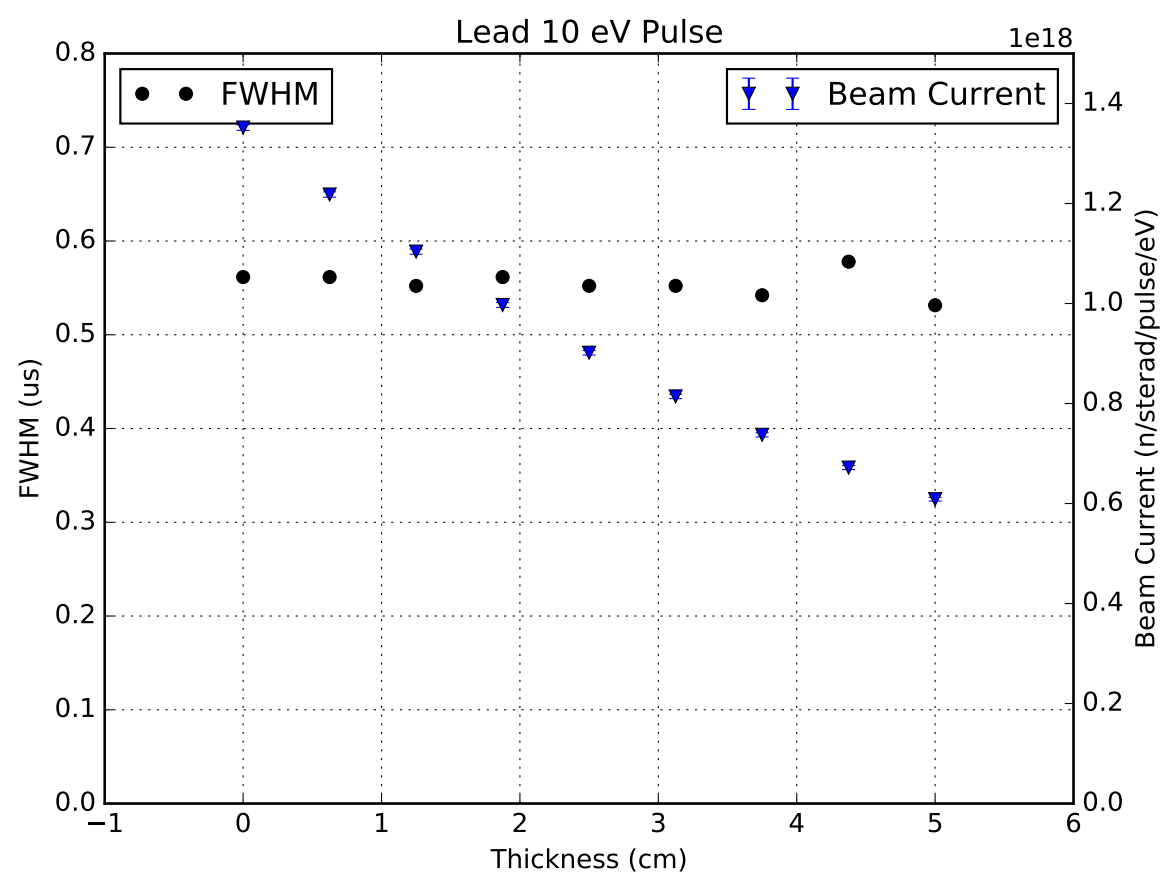

(a) FWHM and Beam Intensity

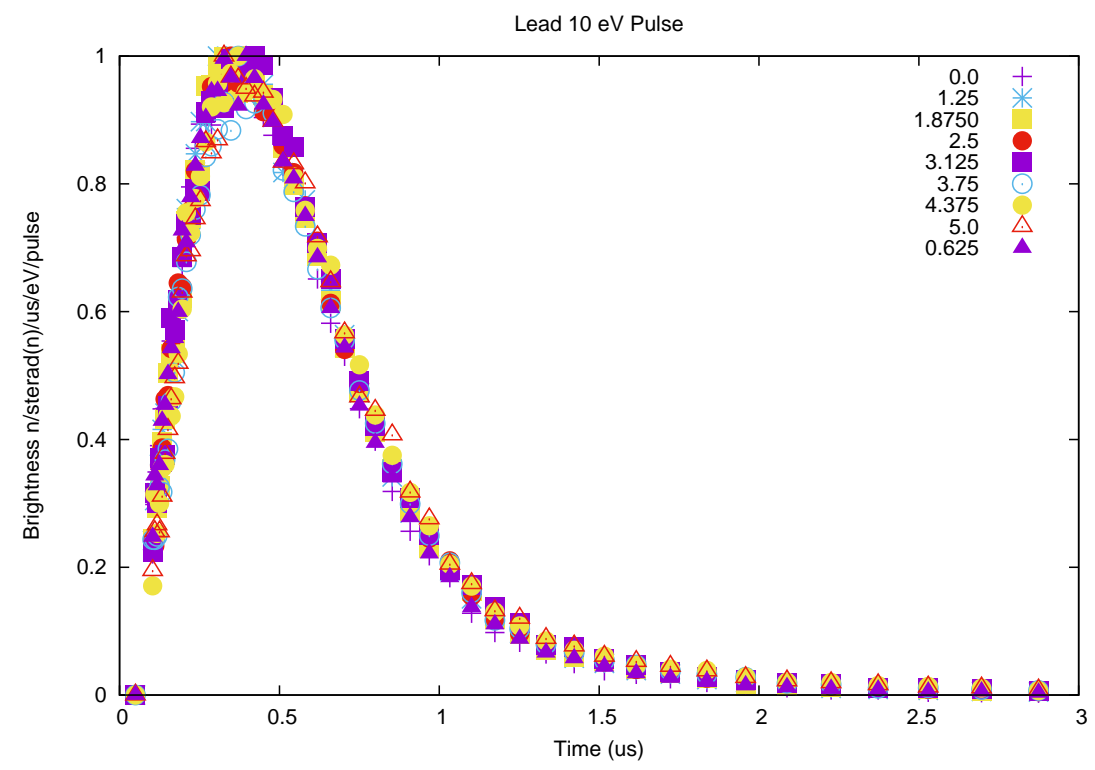

(b) Neutron Pulse Shapes

Figure 17. Analysis of $10 \mathrm{eV}$ Pulse for Lead as the diffusion material 


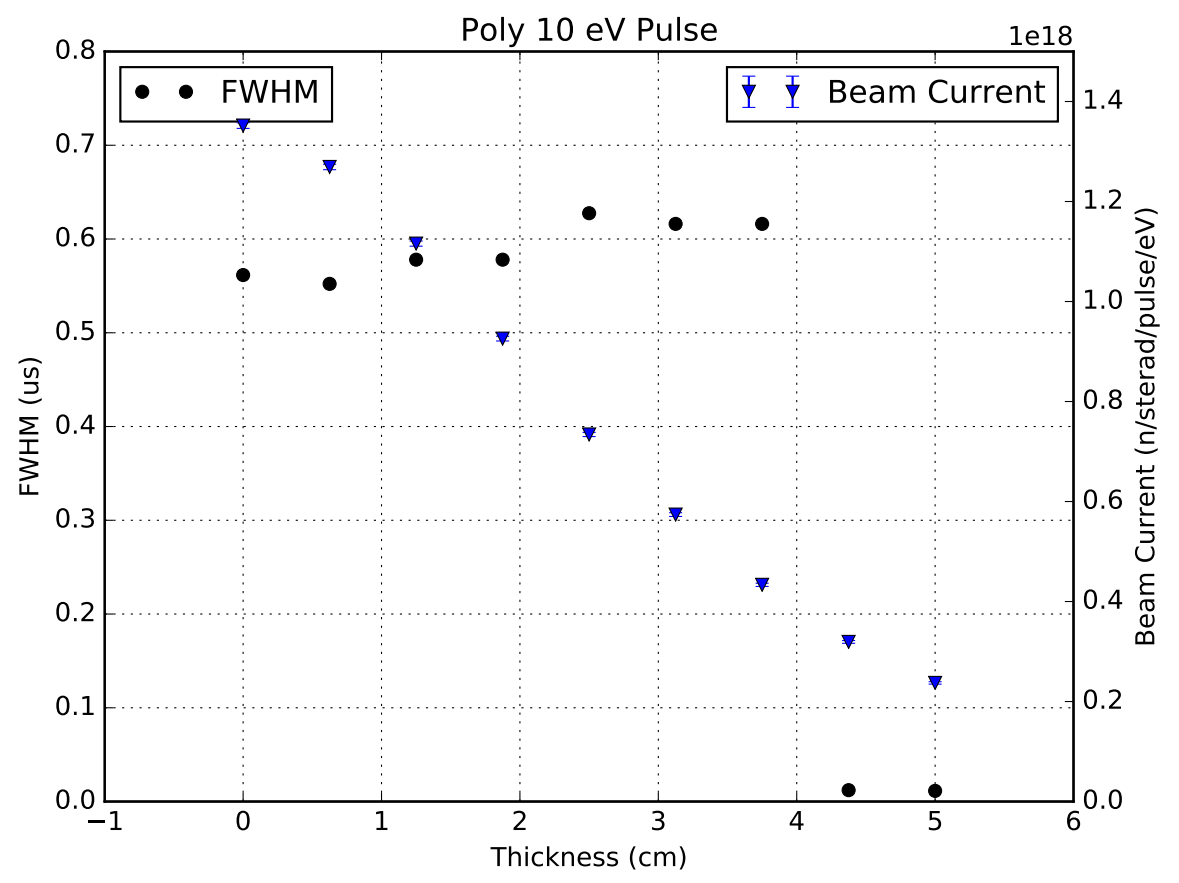

(a) FWHM and Beam Intensity

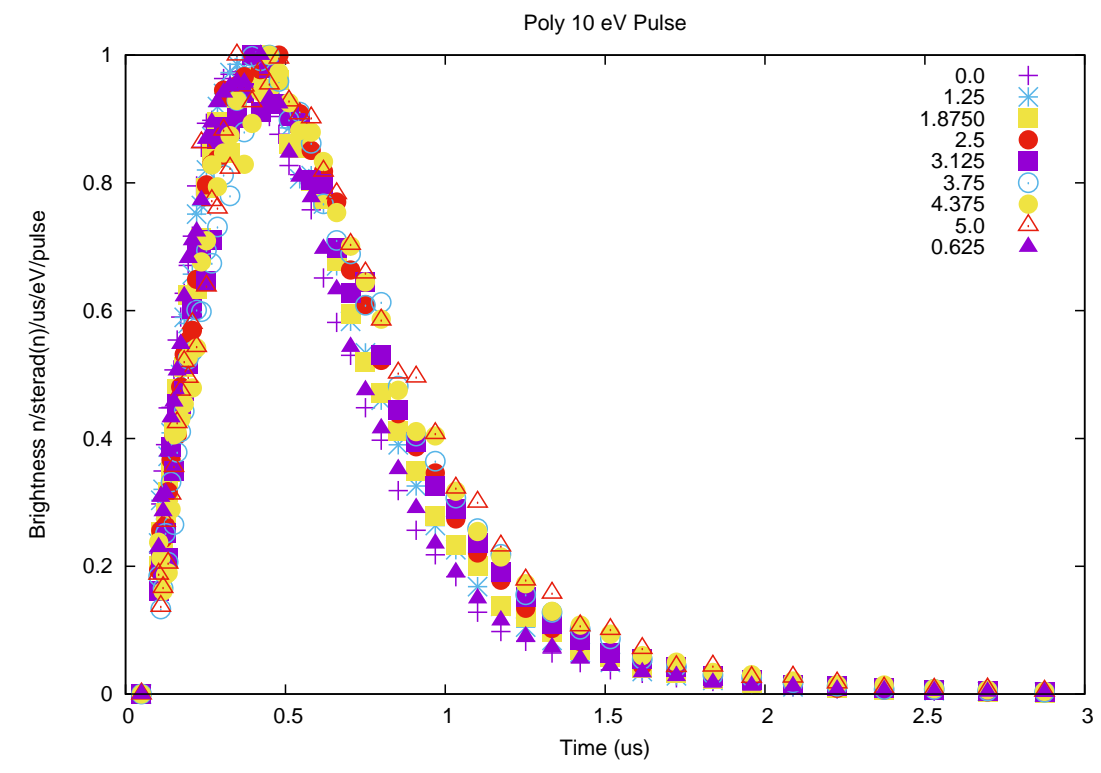

(b) Neutron Pulse Shapes

Figure 18. Analysis of $10 \mathrm{eV}$ Pulse for Polyethylene as the diffusion material 


\subsection{Dakota Results}

In order to match the spatial distribution, we compare the spatial distribution of the slowing-down intensity illuminating the MDF moderator with a comparable quantity as calculated for the SNS STS. Specifically, we compare $\mathrm{F}(\mathrm{x}, \mathrm{y})$, the flux between 0.01 and $10 \mathrm{keV}$ at point $(\mathrm{x}, \mathrm{y})$ as shown in Figure $11 \mathrm{a}$, with $\mathrm{G}(\mathrm{x}, \mathrm{y})$ being the same quantity coming from STS as shown in Figure 11b. We characterize the difference between the $F(x, y)$ and $G(x, y)$ with a reduced $\chi^{2}$ and the $L^{2}$ Norm of the difference between the spatial distributions. The Dakota parameter study routine took the type and thickness of the diffusion material and ran the respective MCNPX simulation and analysis routine. The analysis routine executes the spatial distribution matching routine described above in Section 4.2 and calculated $\chi^{2}$ and $L^{2}$ Norm values for the comparison of the MDF and STS spatial illumination matching. The $\chi^{2}$ value is calculated with Equation 1 in Section 4.2.2. The equation for the calculation of the $L^{2}-$ Norm is given in Equation 2 below.

$$
L^{2} \text { Norm }=\sqrt{\frac{\sum_{i=0}^{N}(F(x, y)-G(x, y))^{2}}{N}}
$$

These values from the Dakota routine are shown below in Figures 19 and 20.

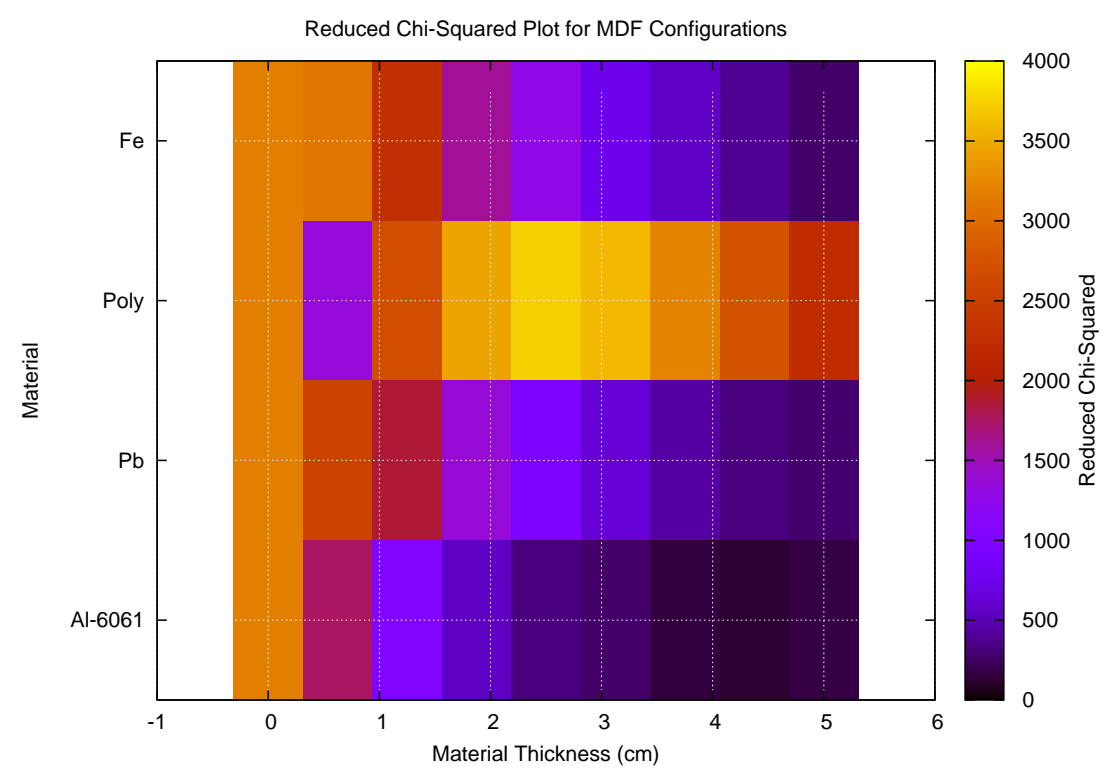

Figure 19. Reduced $\chi^{2}$ Values for the spatial illumination comparison 


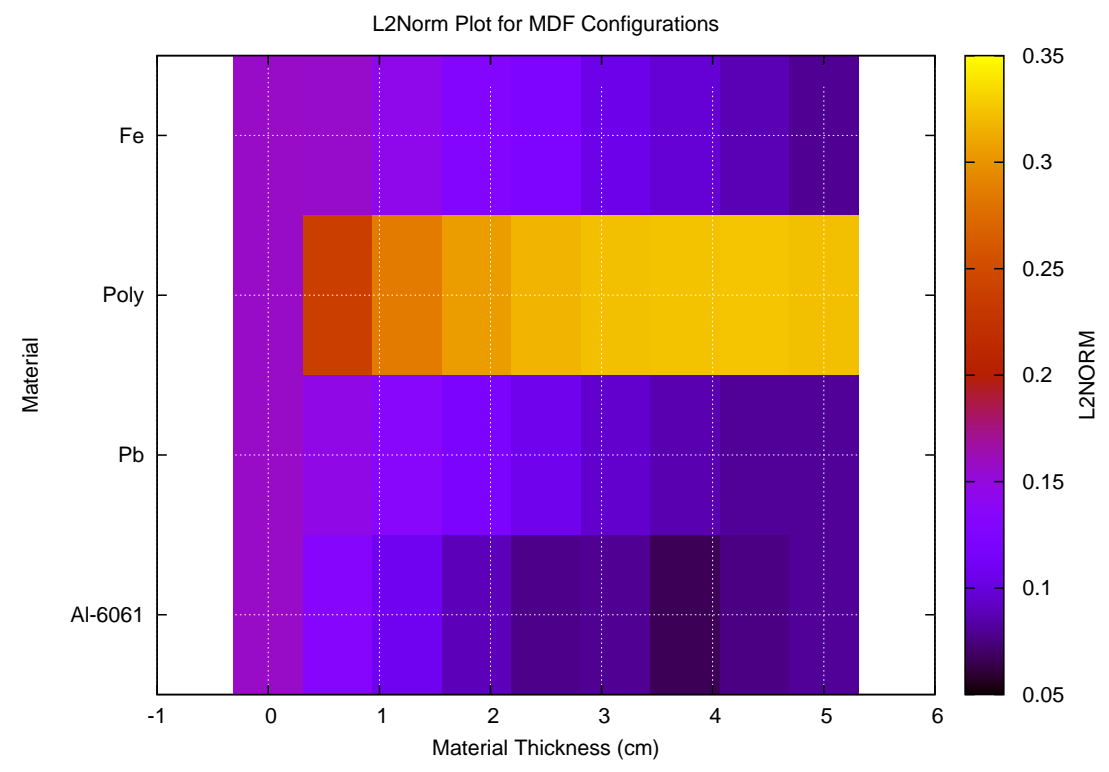

Figure 20. $L^{2}$ Norm Values for the spatial illumination comparison 
The comparison values above show that $5 \mathrm{~cm}$ of Al-6061 provides the closest match of the MDF spatial illumination to the STS spatial illumination. Figure 21 shows the best match of the spatial illumination between the MDF and STS. The comparison of the FWHM and Spectral Beam Intensity above confirm that by adding $5 \mathrm{~cm}$ of Al-6061 between the moderator and source will not drastically alter the neutron emission time distribution. Figures 22 and 23 show cuts through the original spatial distribution (without a diffuser plate) and through the best spatial distribution match ( $5 \mathrm{~cm}$ of $\mathrm{Al}$ as the diffuser plate). The local peaks shown in Figures 22 and 23 are due to rough statistics in the mesh tally used to produce the figures.

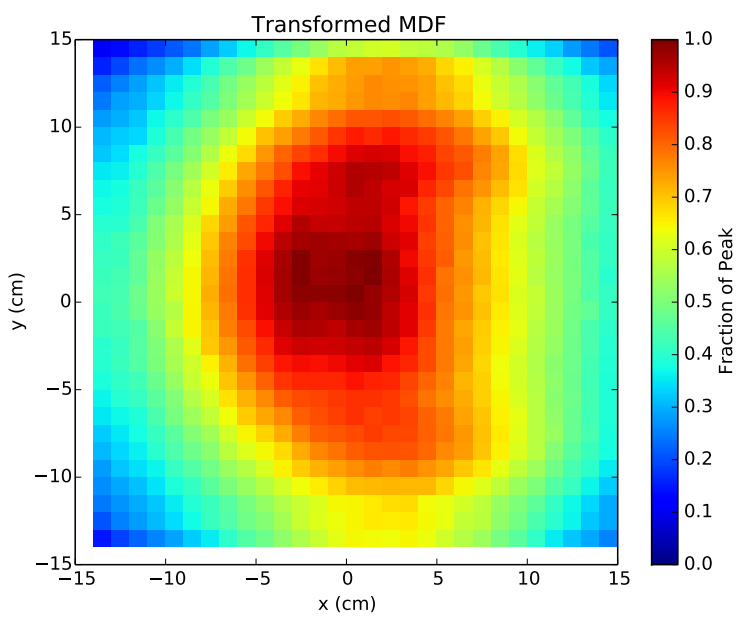

(a) MDF Spatial Distribution

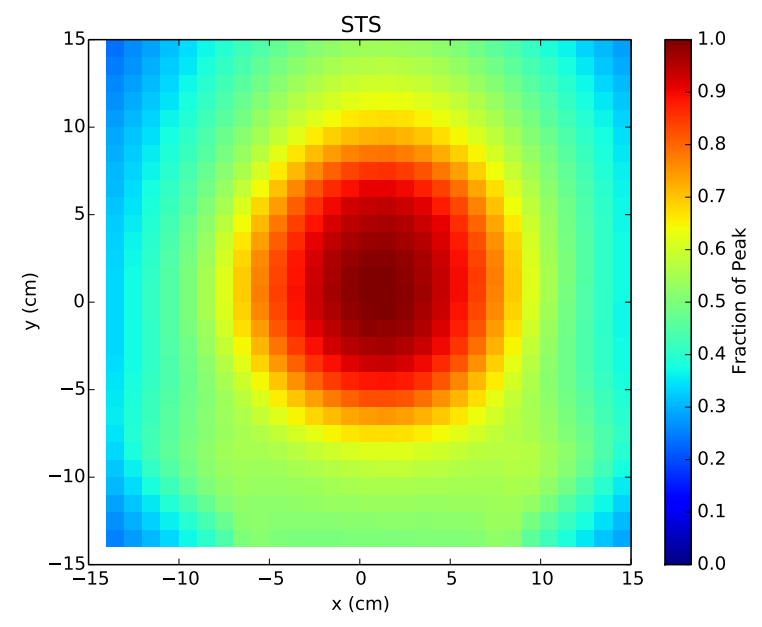

(b) STS Spatial Distribution

Figure 21. Best Match of the spatial illumination between the MDF and STS 


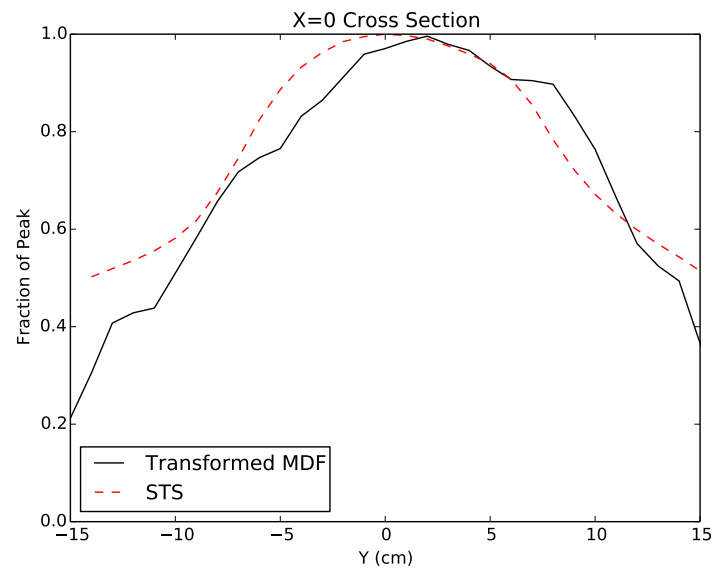

(a) Translated MDF with no diffuser material

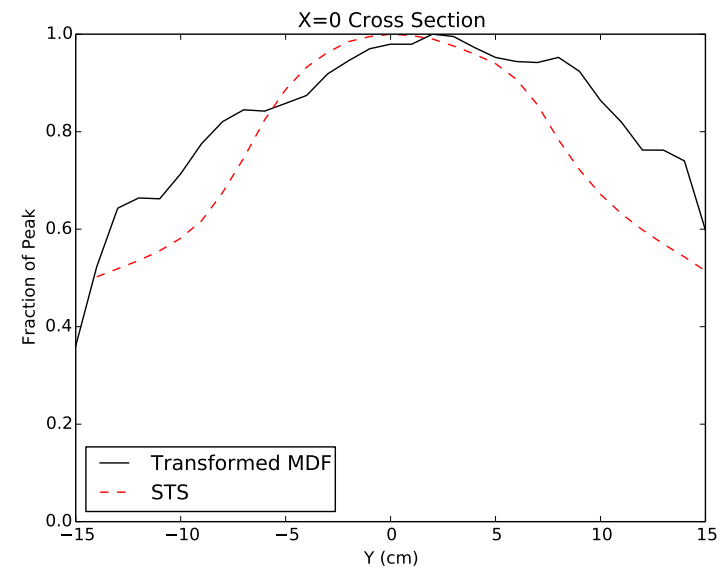

(b) $5 \mathrm{~cm}$ of $\mathrm{Al}$ as diffuser block

\section{Figure 22. $\mathrm{X}=0$ Cross section of spatial distribution}

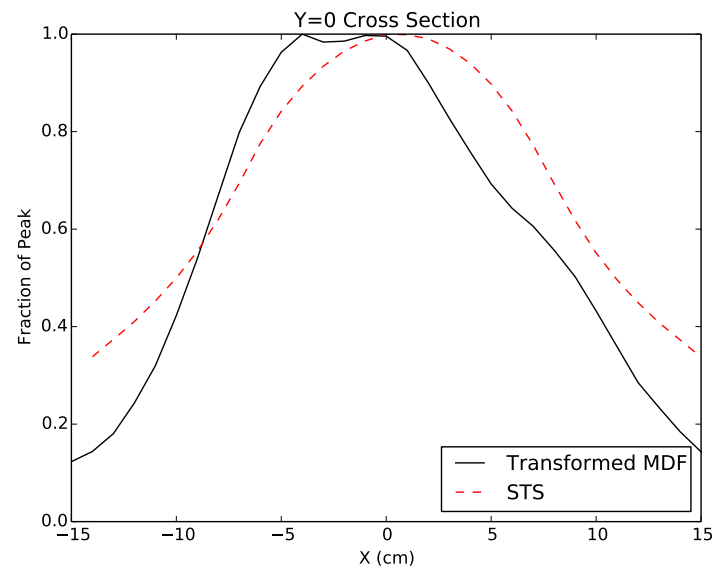

(a) Translated MDF with no diffuser material

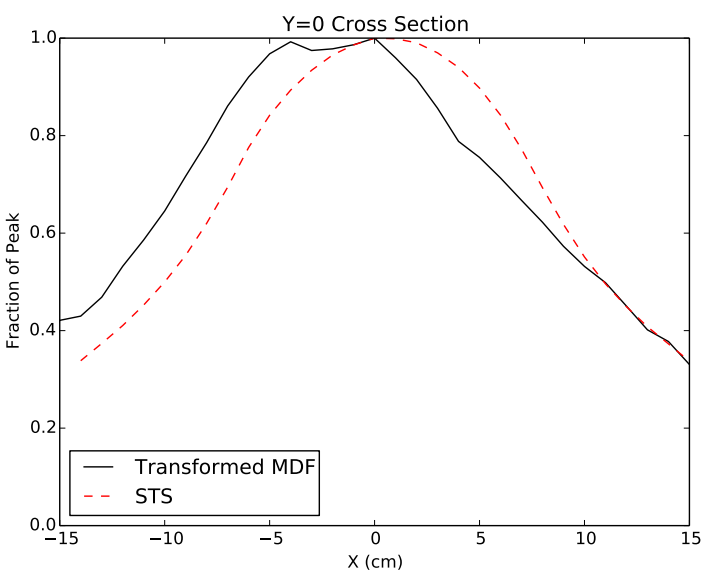

(b) $5 \mathrm{~cm}$ of $\mathrm{Al}$ as diffuser block

Figure 23. $Y=0$ Cross section of spatial distribution 


\section{Shielding Analysis}

The model described in Section 2. was used in a shielding evaluation to verify that the MDF will not have unwanted restrictions to personnel when in operation. The source for this shielding analysis was set at 30 $1-\mu$ s pulses per second at a nominal peak current of $50 \mathrm{~mA}$. The water block shielding surrounding the source/moderator system act as the primary shielding for this analysis. Figures 24, 25 and 26 show the total dose maps (neutron dose plus photon dose) resulting from the shielding analysis. To calculate the neutron and photon dose, ICRP-10 flux-to-dose conversion coefficients were used to convert the flux in a $5.018 \times 5.108 \times 5.018 \mathrm{~cm}$ voxel to dose equivalent rate in units of rem $/ \mathrm{hr}$. The mesh tally spanned $2 \mathrm{~m}$ outside of the MDF target station walls. In Figures 24, 25 and 26, the MDF target station is shown as the white box in the middle of the dose map. The contours in the figures are each of the radiation classification zones at ORNL and show that outside of the Radiation Buffer Area (blue contour in Figures 24, 25 and 26), no restrictions are required for operating with this configuration of the source. For Figures 24 - 35, the XY view corresponds to the view in Figure $3 \mathrm{a}, \mathrm{XZ}$ view corresponds to the view in Figure $3 \mathrm{~b}$, and $\mathrm{YZ}$ view corresponds to a zoomed out view of Figure 5. Figures 27, 28 and 29 show the corresponding relative error of the total dose mesh tally.

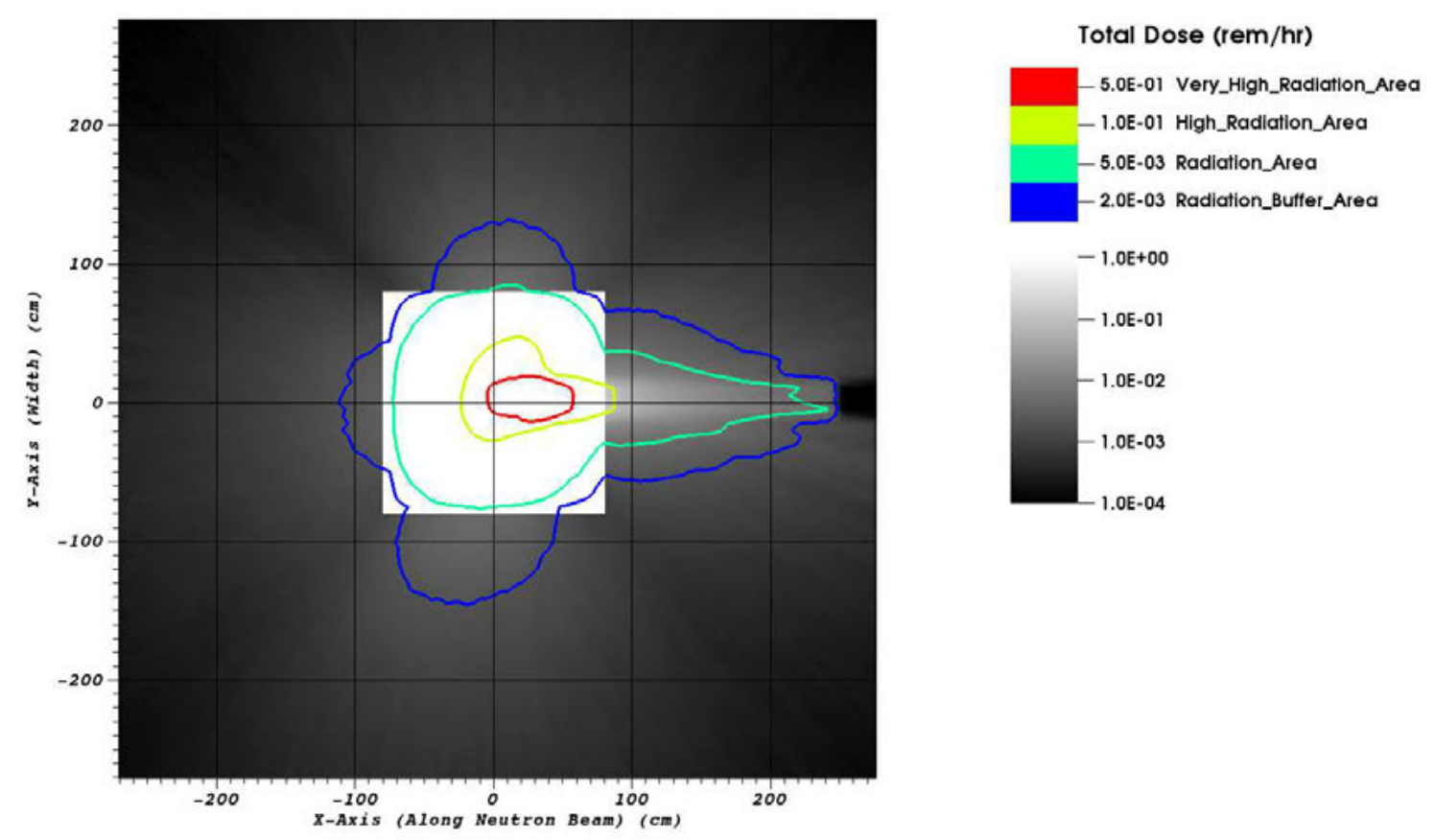

Figure 24. XY View of the dose distribution 


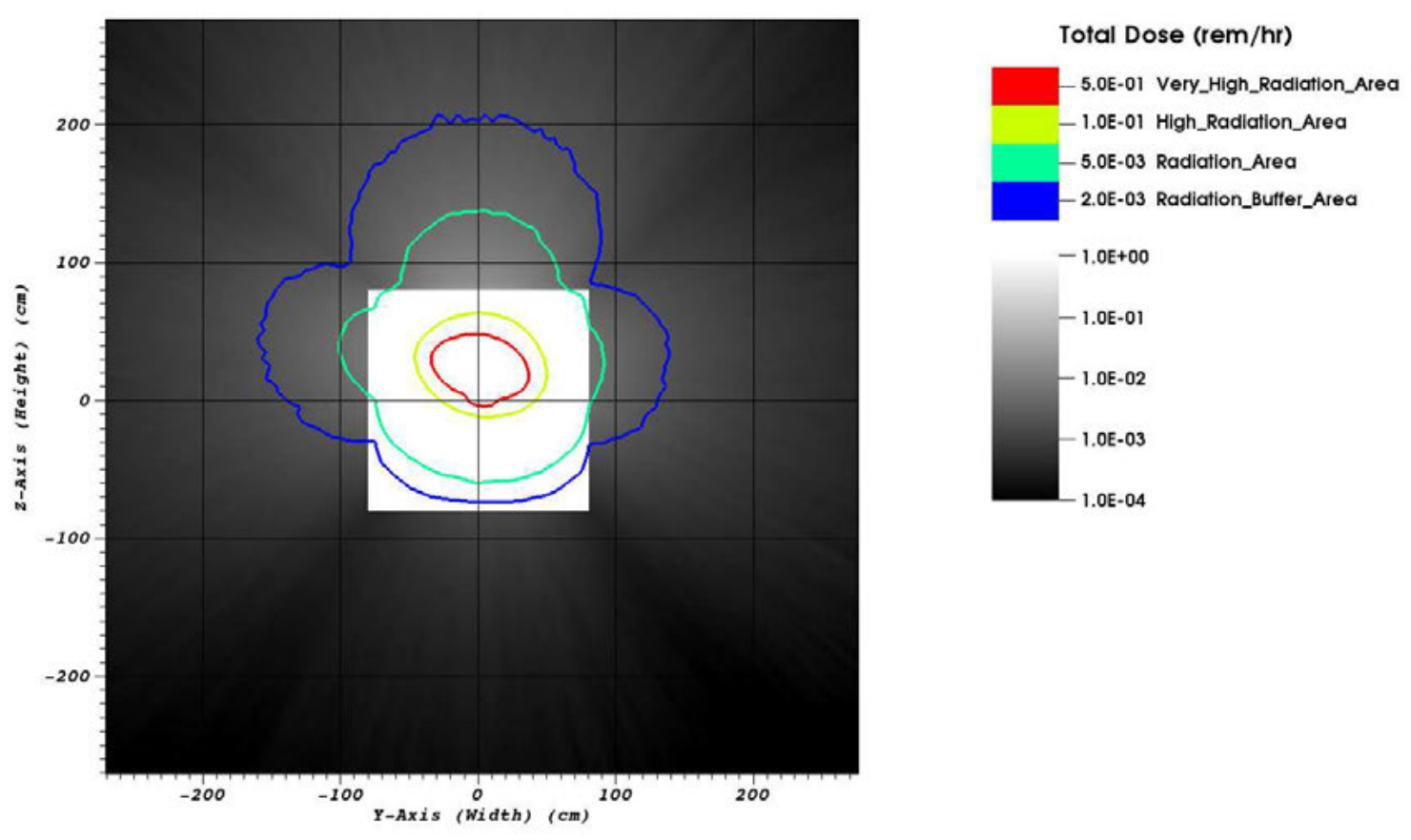

Figure 25. YZ View of the dose distribution

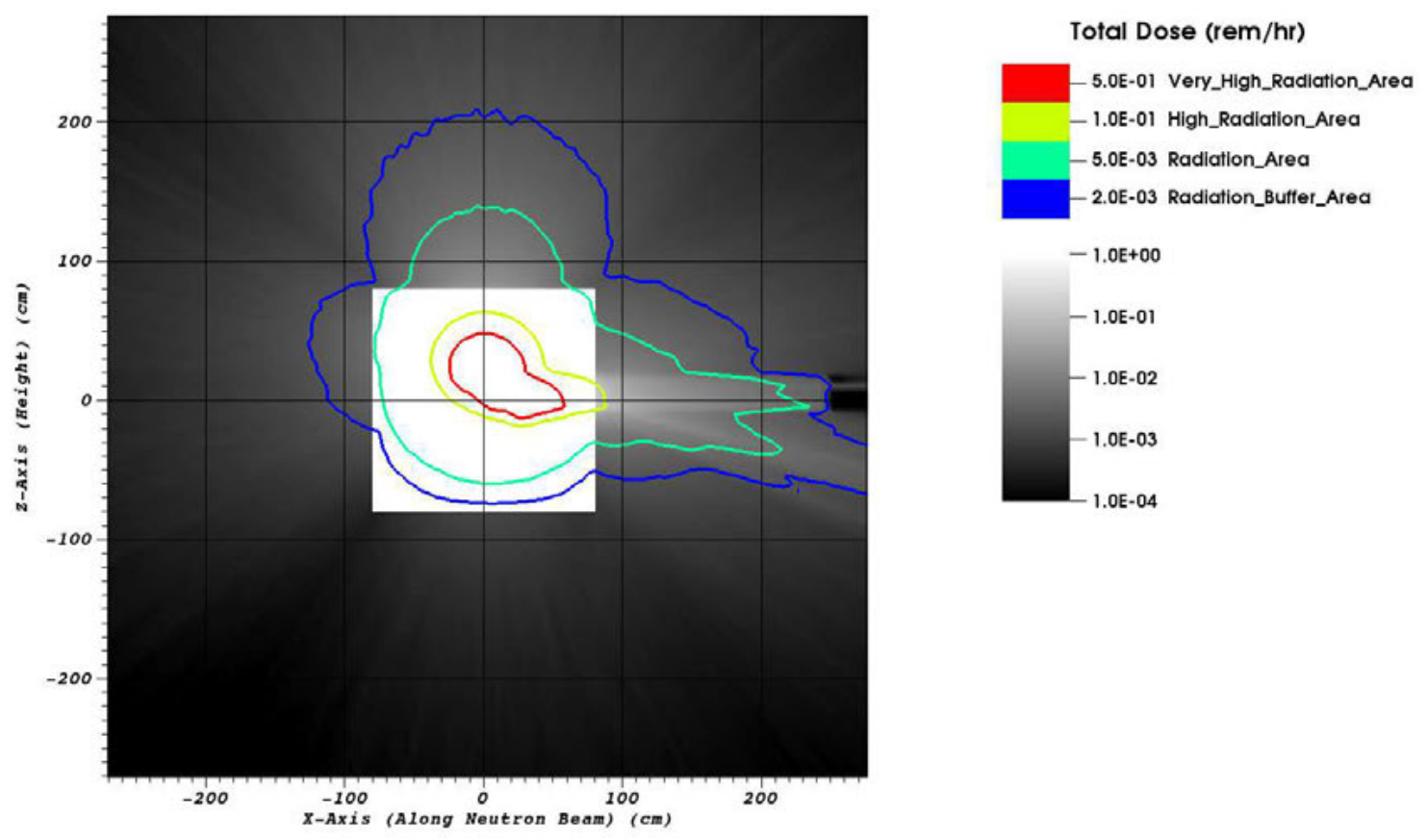

Figure 26. XZ View of the dose distribution 


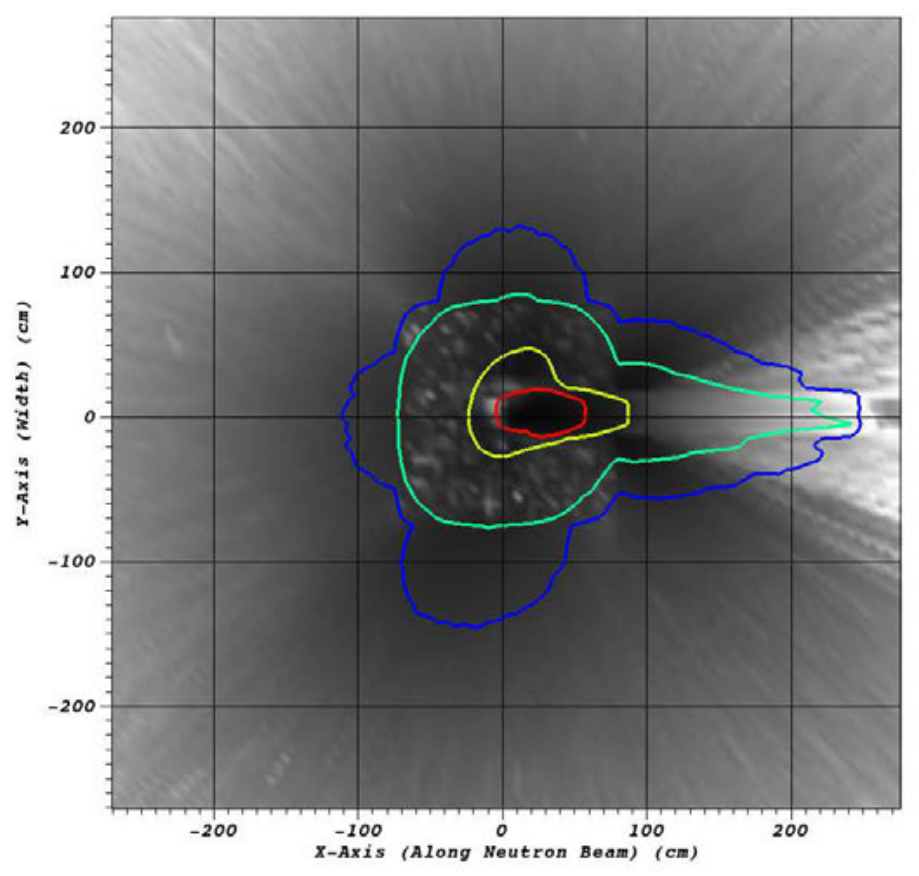

Total Dose (rem/hr)

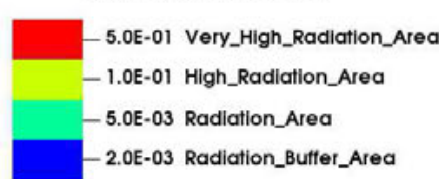

Total Dose Relative Error

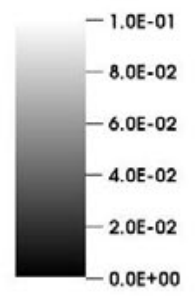

Figure 27. XY View of the dose relative error

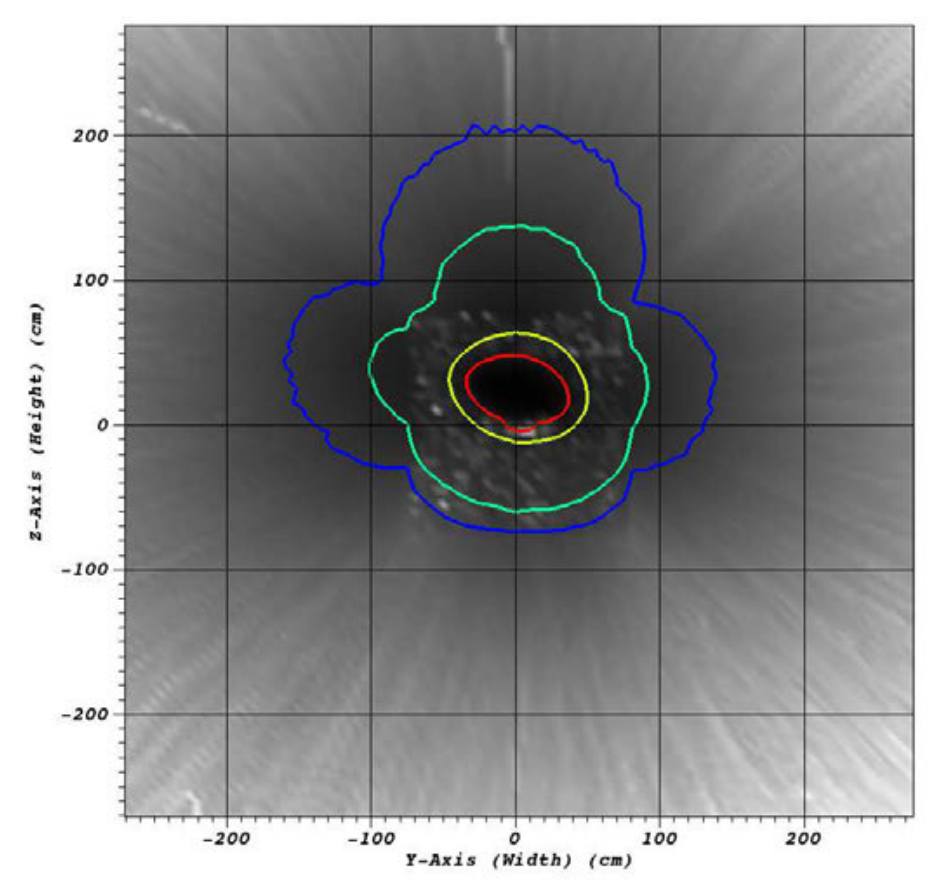

Total Dose (rem/hr)

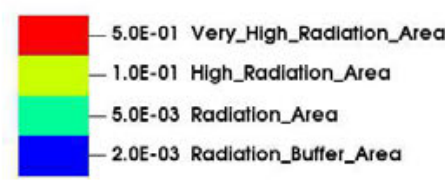

Total Dose Relative Error

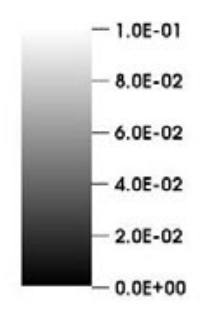

Figure 28. YZ View of the dose relative error 


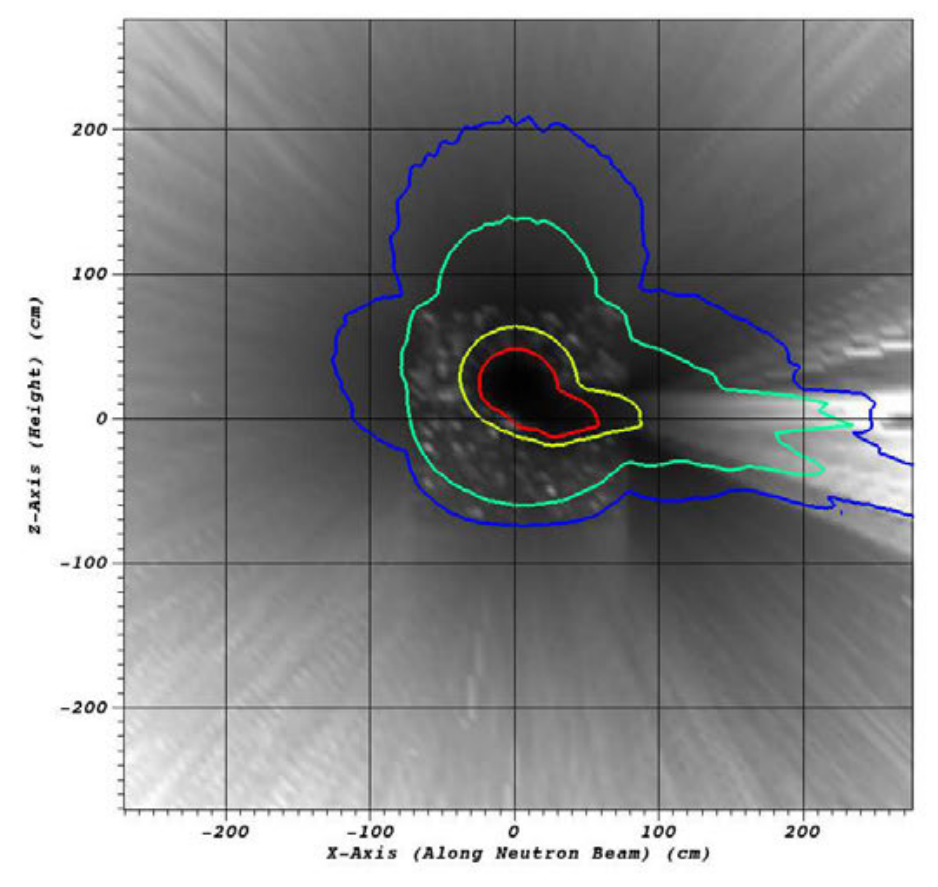

Total Dose (rem/hr)

$-5.0 \mathrm{E}-01$ Very_High_Radiation_Area
$-1.0 \mathrm{E}-01$ High_Radiation_Area
$-5.0 \mathrm{E}-03$ Radiation_Area
$-2.0 \mathrm{E}-03$ Radiation_Buffer_Area

Total Dose Relative Error

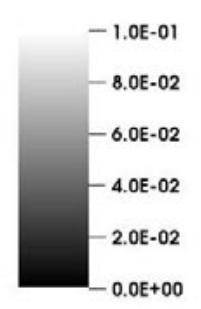

Figure 29. $\mathrm{XZ}$ View of the dose relative error 
Figures 30, 31, 32 show the fraction of neutron dose contributing to the total dose and Figures 33, 34, 35 show the fraction of photon dose contributing to the total dose. These figures clearly show that a majority of the dose outside of the water blocks of the MDF target station is due to photon dose. In order to combat that dose, a study of the dose outside the MDF target station when $20 \mathrm{~g} / \mathrm{L}$ of boron was in the water blocks yielded a reduction of the dose by only a factor of 2-3 and the results of this study are not included in this paper.

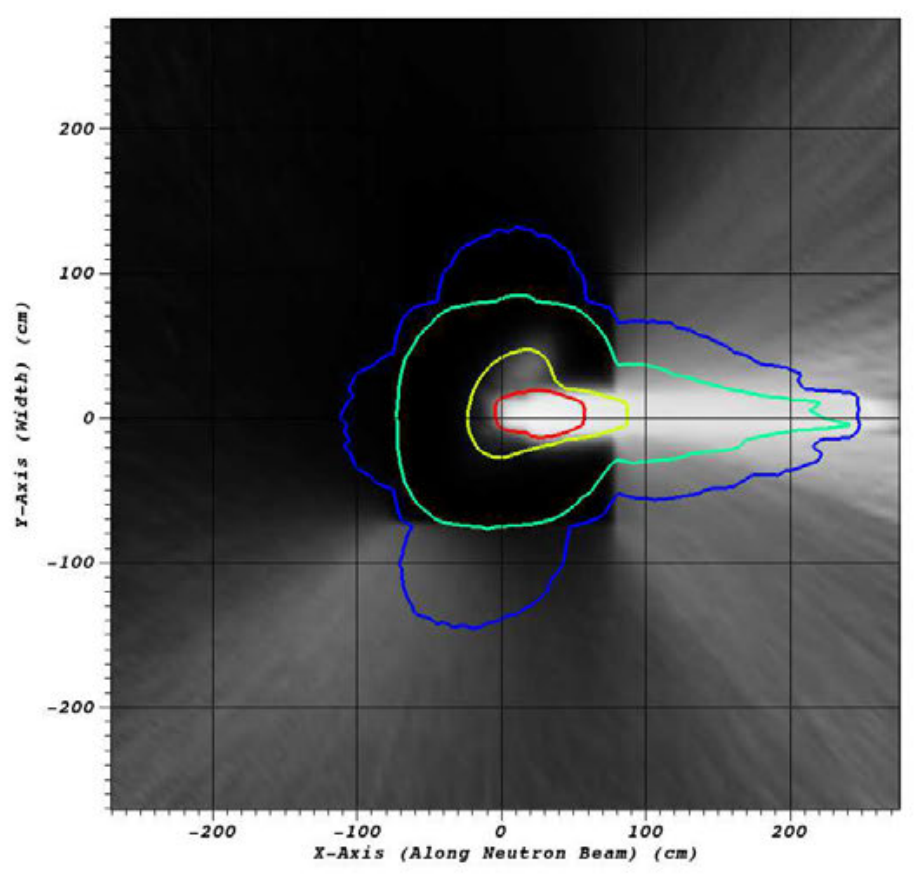

Total Dose (rem/hr)

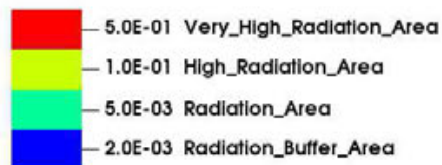

Fractional Neutron/Total Dose

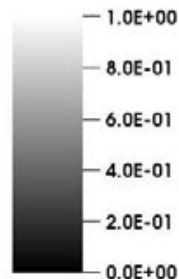

Figure 30. XY View of neutron/total dose 


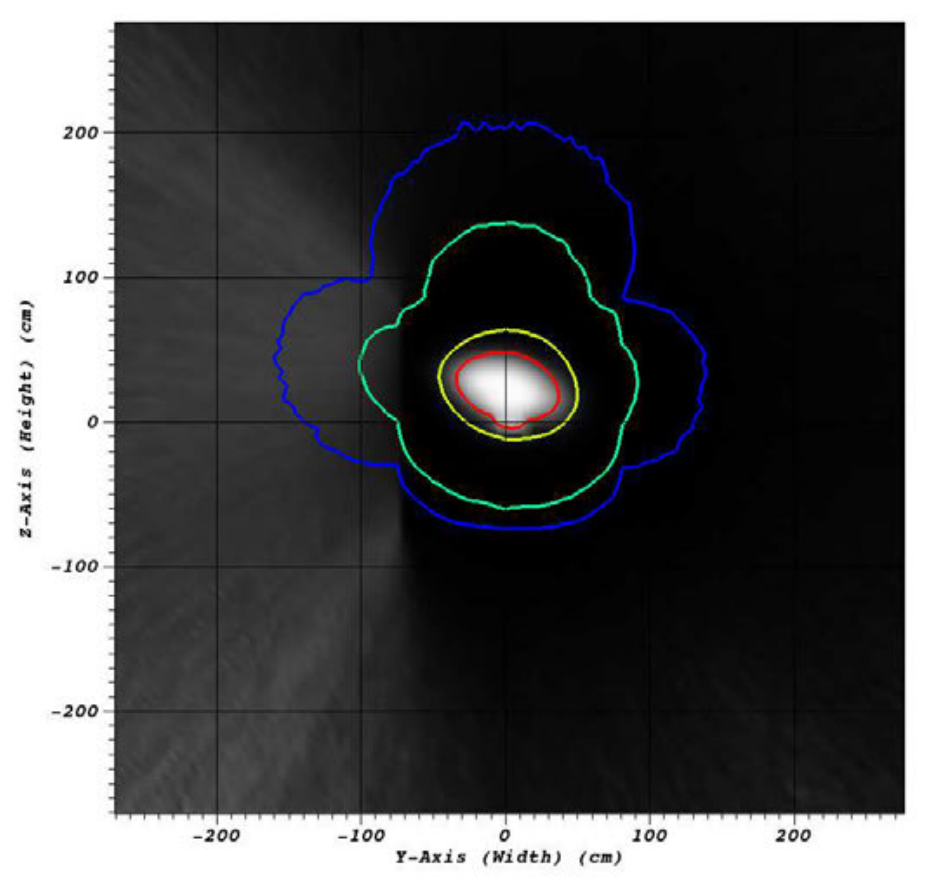

Total Dose (rem/hr)

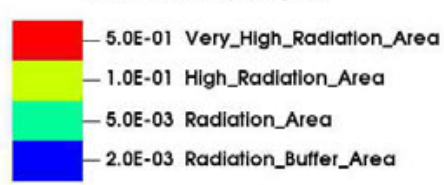

Fractional Neutron/Total Dose

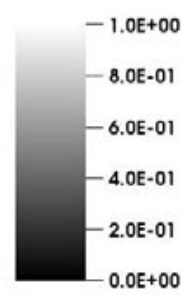

Figure 31. YZ View of neutron/total dose

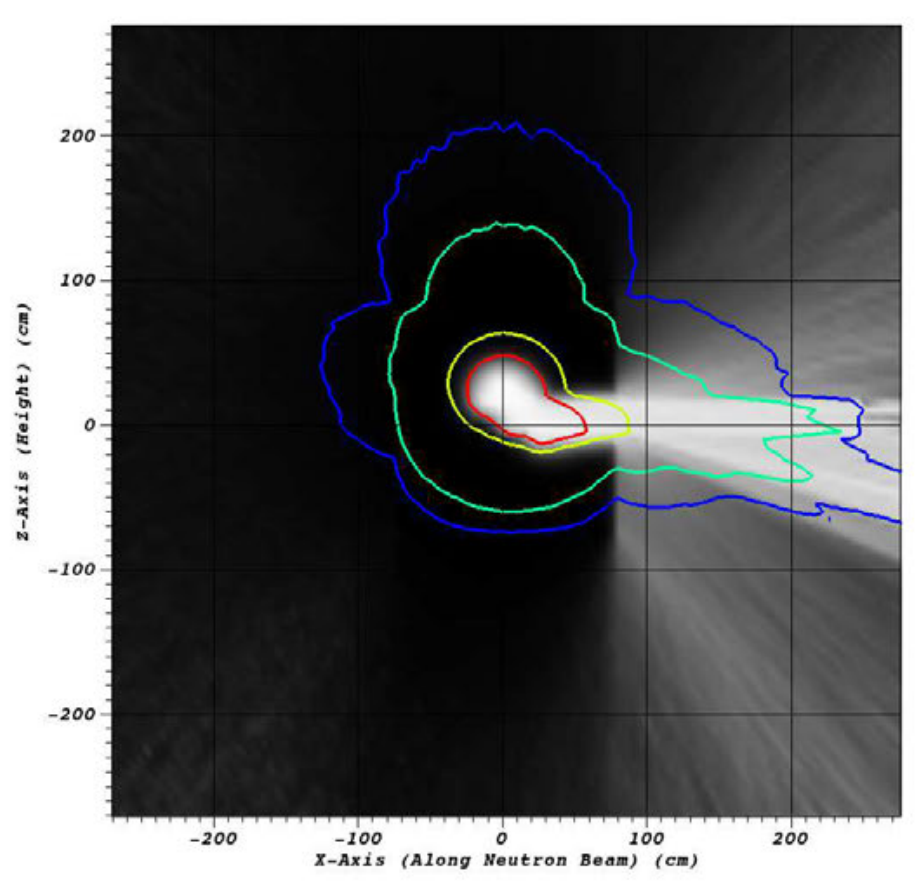

Total Dose (rem/hr)

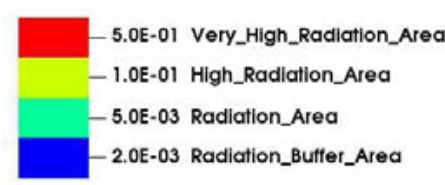

Fractional Neutron/Total Dose

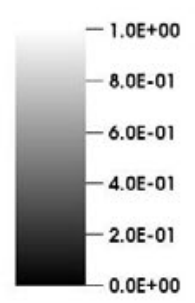

Figure 32. XZ View of neutron/total dose 


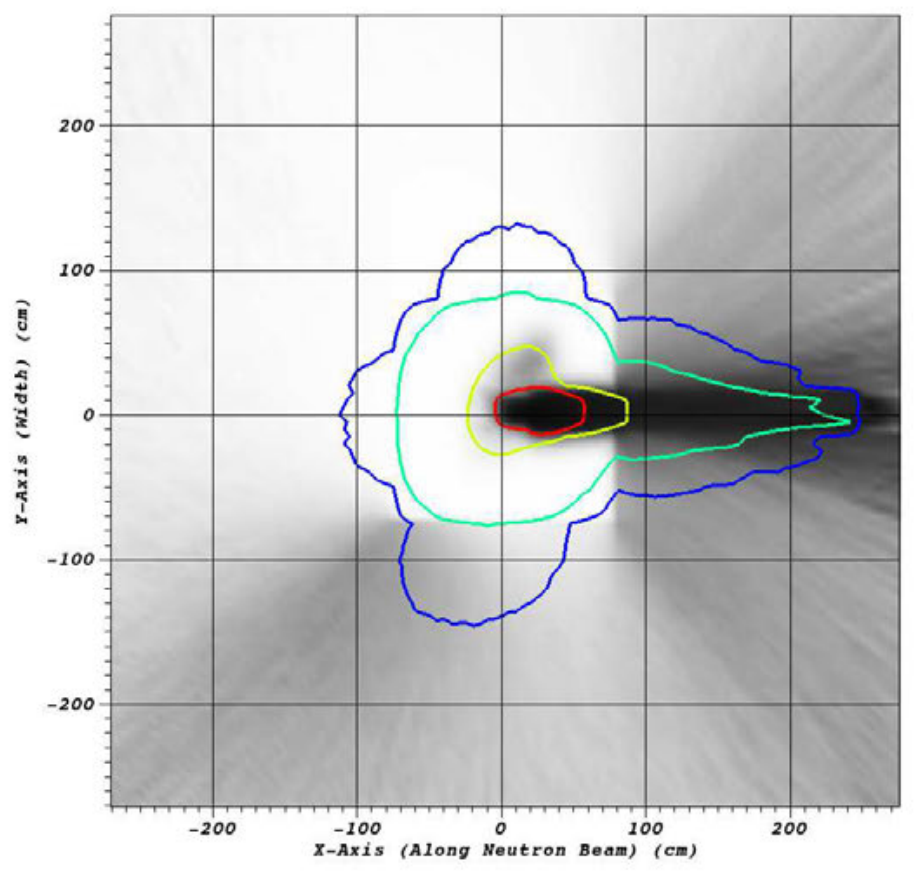

\section{Total Dose (rem/hr)}

-5.0E-01 Very_High_Radiation_Area

- 1.0E-01 High_Radiation_Area

-5.0E-03 Radiation_Area

-2.0E-03 Radiation_Buffer_Area

\section{Fractional Photon/Total Dose}

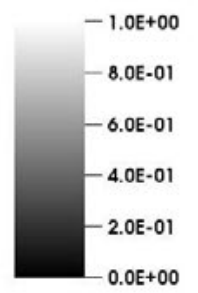

Figure 33. XY View of photon/total dose

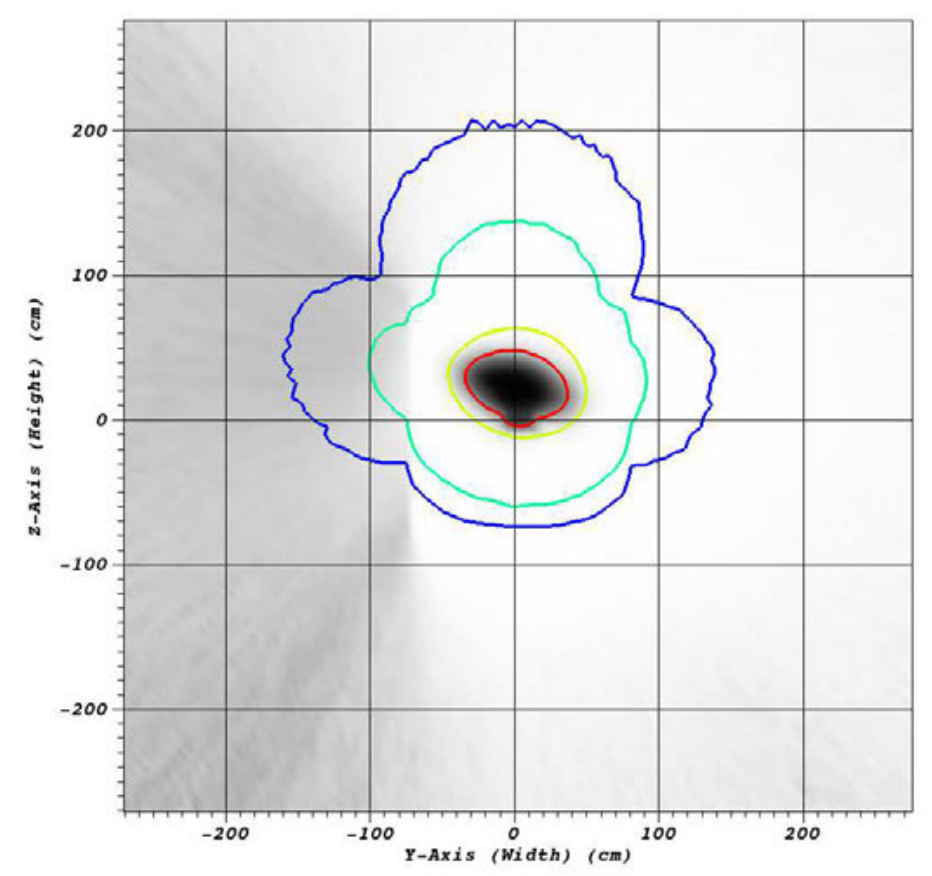

Total Dose (rem/hr)

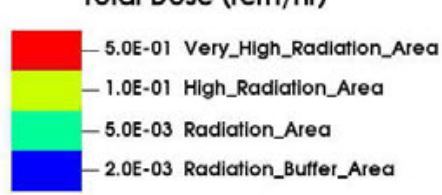

Fractional Photon/Total Dose

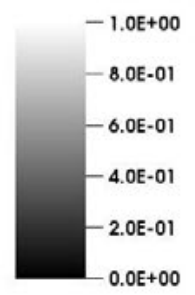

Figure 34. YZ View of photon/total dose 


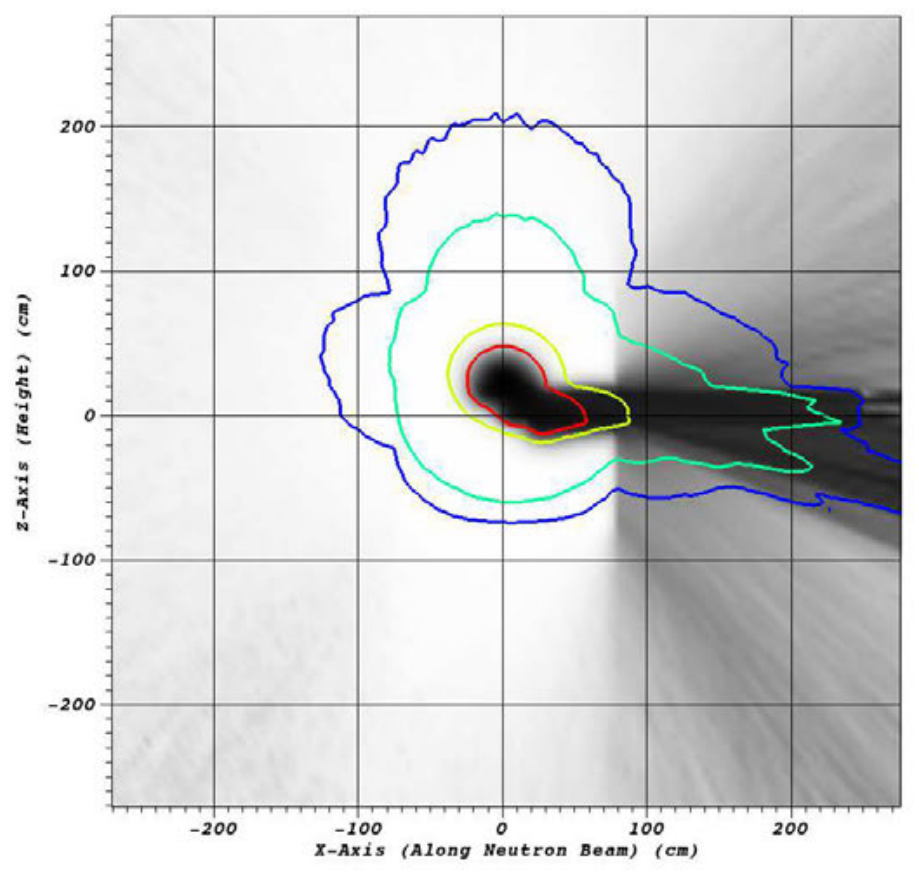

Total Dose (rem/hr)

-5.0E-01 Very_High_Radiation_Area

- 1.0E-01 High_Radiation_Area

-5.0E-03 Radiation_Area

-2.0E-03 Radiation_Buffer_Area

Fractional Photon/Total Dose

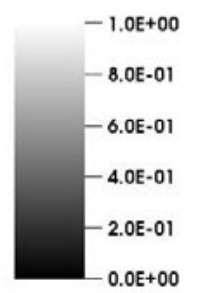

Figure 35. XZ View of photon/total dose 


\section{Conclusions}

The design of the MDF target station will allow next generation moderators intended for use on the STS to be characterized by a prototypic moderator illumination similar to the STS. In order to improve the prototypic moderator illumination, a $5 \mathrm{~cm} \mathrm{Al-6061} \mathrm{diffuser} \mathrm{plate} \mathrm{is} \mathrm{added} \mathrm{between} \mathrm{the} \mathrm{target} \mathrm{plate} \mathrm{and} \mathrm{the}$ moderator. Various diffuser materials and material thicknesses were analyzed with an elaborate Dakota parameter study. Many tools were used to accelerate the neutron transport of the MDF model, quantify the similarities between MDF and STS spatial distributions, and verify that the addition of a diffuser plate would not alter the neutron emission time distribution. Much work was done optimizing the weight windows generated by ADVANTG. The phase correlation image translation technique was used to translate the spatial profiles in order for a $\chi^{2}$ value to be calculated. A fitting routine using the LMFIT Python package was developed to verify that the neutron emission time distribution was not changed by the addition of the diffuser plate, but ultimately was replaced by a comparison of the FWHM and beam current. Finally, a shielding analysis confirmed that the shielding incorporated into the MDF target station was sufficient to classify the zone within $2 \mathrm{~m}$ from the wall of the MDF target station a Radiation Buffer Area. 


\section{References}

[1] J. M. Carpenter, "Pulsed Spallation Neutron Sources for Slow-Neutron Scattering," Nuclear Instruments $\mathcal{E}$ Methods, vol. 145, no. 1, pp. 91-113, 1977.

[2] D. Pelowitz, “MCNPX User's Manual,” tech. rep., LANL, April 2011.

[3] S. W. Mosher, S. R. Johnson, A. M. Bevill, A. M. Ibrahim, C. R. Daily, T. M. Evans, J. C. Wagner, J. O. Johnson, and R. E. Grove, "ADVANTG- An Automated Variance Reduction Parameter Generator," tech. rep., Oak Ridge National Laboratory, August 2015.

[4] B. M. Adams, M. S. Ebeida, M. S. Eldred, J. D. Jakeman, K. A. Maupin, J. A. Monschke, L. P. Swiler, J. A. Stephens, D. M. Vigil, T. M. Wildey, W. J. Bohnhoff, K. R. Dalbey, J. P. Eddy, R. W. Hooper, K. T. Hu, P. D. Hough, E. M. Ridgway, and A. Rushdi, "Dakota, A Multilevel Parallel Object-Oriented Framework for Design Optimization, Parameter Estimation, Uncertainty Quantification, and Sensitivity Analysis," tech. rep., Sandia National Laboratories, July 2014.

[5] S. Ikeda and J. M. Carpenter, "Wide-energy-range, high-resolution measurements of neutron pulse shapes of polyethylene moderators," Nuclear Instruments and Methods in Physics Research Section A: Accelerators, Spectrometers, Detectors and Associated Equipment, vol. 239, pp. 536-544, September 1985.

[6] M. Newville and F. Stensitzki, "LMFIT Non-Linear Least-Squares Minimization and Curve-Fitting for Python," March 2016.

[7] R. R. Borchers and C. H. Poppe, "Neutrons from Proton Bombardment of Lithium," Physical Review, vol. 129, pp. 2679-2683, March 1963.

[8] F. B. Brown, J. E. Sweezy, and R. B. Hayes, "Monte Carlo Parameter Studies and Uncertainty Analysis with MCNP5," tech. rep., LANL, 2004.

[9] B. J. Micklich and E. B. Iverson, "Calculation of pulse shapes for reentrant moderators," 2003.

[10] T. McClanahan, F. Gallmeier, and E. Iverson, "Acceleration Techniques using ADVANTG for the MDF Design and Optimization," in Transactions of the American Nuclear Society (R. Michal, B. Tompkins, and D. Cacuci, eds.), vol. 114, (New Orleans, LA), pp. 961-4, American Nuclear Society, 2016.

[11] J. K. Zhao, K. W. Herwig, J. L. Robertson, F. X. Gallmeier, and B. W. Riemer, "Instrument performance study on the short and long pulse options of the second Spallation Neutron Source target station," Review of Scientific Instruments, vol. 84, no. 10, 2013.

[12] J. K. Zhao, J. L. Robertson, K. W. Herwig, F. X. Gallmeier, and B. W. Riemer, "Optimizing moderator dimensions for neutron scattering at the Spallation Neutron Source," Review of Scientific Instruments, vol. 84, no. 12, 2013.

[13] F. Gallmeier, E. Iverson, W. Lu, D. Baxter, G. Muhrer, and S. Ansell, "Introducing single-crystal scattering and optical potentials into MCNPX: Predicting neutron emission from a convoluted moderator," Nuclear Instruments and Methods in Physics Research Section A: Accelerators, Spectrometers, Detectors and Associated Equipment, vol. 814, pp. 39-49, 2016. 
[14] E. B. Iverson, P. D. Ferguson, F. X. Gallmeier, and I. I. Popova, "Performance characteristics of SNS neutron beams," Transactions of the American Nuclear Society, vol. 89, pp. 673-674, 2003.

[15] R. K. Crawford and E. B. Iverson, "Matching source parameters to neutron-scattering-instrument requirements at spallation neutron sources," Transactions of the American Nuclear Society, vol. 79, pp. 392-393, 1998.

[16] B. J. Micklich and E. B. Iverson, "Calculation of pulse shapes for reentrant moderators," in Nuclear Mathematical and Computational Sciences: A Century in Review, A Century Anew, 2003.

Proceedings of a conference held in Gatlinburg, Tennessee, April 6-11, 2003. Available on CD-ROM from the American Nuclear Society, LaGrange Park, IL.

[17] E. B. Iverson and J. M. Carpenter, "Cryogenic moderators on pulsed neutron sources: simulations and measurements," in Proceedings of the 2nd International Topical Meeting on Nuclear Applications of Accelerator Technology, AccApp ‘98, pp. 46-58, 1998.

[18] E. B. Iverson, P. D. Ferguson, F. X. Gallmeier, and B. D. Murphy, "The Spallation Neutron Source high power target station moderator performance: calculations and studies," Journal of Neutron Research, vol. 11, pp. 83-91, 2003.

[19] I. Remec, F. X. Gallmeier, M. J. Rennich, T. J. McManamy, and W. Lu, "Neutronics Analyses for the SNS Second Target Station," in In ANS MC2015 - the Proceedings of the Joint International Conference on Mathematics and Computation $(M \mathcal{E} C)$, Supercomputing in Nuclear Applications (SNA) and the Monte Carlo (MC) Method, vol. 4, pp. 2713-2726, American Nuclear Society, 2015.

[20] B. J. Micklich, E. B. Iverson, and J. M. Carpenter, "Comparison of calculated and measured neutron intensities for the IPNS cryogenic methane moderators," in Proceedings of the ICANS-XVI The 16th Meeting of the International Collaboration on Advanced Neutron Sources, pp. 719-733, 2003.

[21] M. Guizar-Sicairos, S. T. Thurman, and J. R. Fienup, "Efficient subpixel image registration algorithms,” Optics Letters, vol. 33, p. 156, January 2008.

[22] B. Reddy and B. Chatterji, "An FFT-based technique for translation, rotation, and scale-invariant image registration," IEEE Transactions on Image Processing, vol. 5, no. 8, pp. 1266-1271, 1996.

[23] E. De Castro and C. Morandi, "Registration of Translated and Rotated Images Using Finite Fourier Transforms," IEEE Transactions on Pattern Analysis and Machine Intelligence, vol. PAMI-9, pp. 700-703, September 1987.

[24] E. Jones, T. Oliphant, P. Peterson, and Others, "SciPy: Open source scientific tools for Python,” 2001.

[25] F. X. Gallmeier, "SNS Source Descriptions for Use with MCSTAS," tech. rep., Oak Ridge National Laboratory, October 2010.

[26] E. B. Iverson, P. D. Ferguson, F. X. Gallmeier, and I. I. Popova, "Detailed SNS neutronics calculations for scattering instrument design: SCT configuration," tech. rep., Oak Ridge National Laboratory, July 2002.

[27] R. A. Robinson and J. M. Carpenter, "On the use of switch functions in describing pulsed neutron moderators," Nuclear Instruments and Methods in Physics Research Section A: Accelerators, Spectrometers, Detectors and Associated Equipment, vol. 307, pp. 359-365, October 1991. 
[28] H. J. Motulsky and L. A. Ransnas, "Fitting curves to data using nonlinear regression: a practical and nonmathematical review.," FASEB journal : official publication of the Federation of American Societies for Experimental Biology, vol. 1, pp. 365-74, November 1987. 
APPENDIX A. Details of Fitting the Ikeda Carpenter Function 


\section{APPENDIX A. Details of Fitting the Ikeda Carpenter Function}

\section{Fitting the Ikeda-Carpenter Function}

IC function has long been used to characterize the emission time distribution of neutrons coming from pulsed-source moderator surfaces $[5,25,26,27]$. Equation 3 shows the IC function as written in the referenced paper:

$\psi(v, t)=\frac{\alpha}{2}\left\{(1-R)\left(\alpha\left(t-t_{0}\right)\right)^{2} e^{-\alpha\left(t-t_{0}\right)}+2 R \frac{\alpha^{2} \beta}{(\alpha-\beta)^{3}} \times\left[e^{-\beta\left(t-t_{0}\right)}-e^{-\alpha\left(t-t_{0}\right)}\left(1+(\alpha-\beta)\left(t-t_{0}\right)+\frac{1}{2}(\alpha-\beta)^{2}\left(t-t_{0}\right)^{2}\right)\right]\right\}$

for $t>0$ where $\alpha=v \Sigma$, $\mathrm{R}$ is the ratio of the area of the second term to the total area, $\beta$ is the time constant for the storage term and $\Sigma$ is the total macroscopic cross section [5]. The IC function is also only a spectral shape function and therefore integrates to unity. The IC function consists of two components: the slowing down term and storage term. The slowing down term represents the neutrons that are emitted while they are slowing down but not completely thermalized, and the storage term accounts for the neutrons that are emitted after they have been thermalized.

The IC function can be used to fit measured or simulated time emission distributions from pulsed-source moderators by fitting the following four parameters:

- $\alpha=v \Sigma$ - where $\Sigma$ is the macroscopic cross section and $v$ is the velocity of the neutron

- $\beta$ - the time constant of the slowing down distribution.

- $R$ - the ratio the storage term over the slowing down term. $R$ gives an indication of the prominence of the tail of the distribution.

- $t_{0}$ - the time delay of the neutron flight time between the detector and the emission surface.

In order to fit the neutron time emission distributions for neutron energies between $0.01 \mathrm{meV}$ and $100 \mathrm{eV}$, the fitting routine developed to fit the IC function with the LMFIT suite of tools incorporated iterative fitting. Iterative fitting is when the user supplies initial guesses for the first neutron emission time distribution at the highest energy in the dataset, and then the routine calculates the best parameter set for that highest energy and uses that parameter set as the initial guess for the next lowest energy in the dataset. For example, to start the fitting routine, the user supplies the initial guesses for the parameters at $100 \mathrm{eV}$. The fitting routine then calculates the best parameter set for $100 \mathrm{eV}$ and uses the optimized parameter set at $100 \mathrm{eV}$ as the initial guess for the search for the best parameter set at $99 \mathrm{eV}$ and so on. Also, each neutron time emission distribution was fit in two rounds. The first round of fitting allows all four variables to vary in order to acquire an estimate for $\beta$ and $t_{0}$. The second round of fitting holds $\beta$ and $t_{0}$ constant and fits $\mathrm{R}$ and $\alpha$.

Along with iterative fitting and fitting in rounds, the fitting routine also incorporates three different weighting schemes to be used in the non-linear least-squares fitting: 
- Fitting Strategy 1: $W_{i}=\frac{x_{i}}{\sigma_{i}^{2}}$

- Fitting Strategy 2: $W_{i}=\frac{x_{i}^{2}}{\sigma_{i}^{2}}$

- Fitting Strategy 3: Fit the logarithm of the data without weights

where $x_{i}$ is the value that is being fitted and $\sigma_{i}$ is the variance of the value being fitted. Originally, the weight was set to $W_{i}=\frac{1}{\sigma^{2}}$ based on a literature review but the results were not satisfactory [28]. Therefore the three weighting schemes above were devised. As the fitting routine fits the spectra from the highest to the lowest energy, all three weighting schemes are used to calculate three different parameter sets. Three chi-squared statistics are calculated using these three parameter sets and the parameter set with the lowest chi-squared value is chosen as the optimal parameter set.

Originally, the parameters in the below figures were fit to analytical functions as described in the referenced paper [5] and shown below in Equations 4,5 and 6.

$$
\alpha=v \sqrt{S_{1}^{2}+S_{2}^{2} \lambda^{2}}
$$

where $S_{1}$ and $S_{2}$ are the fitted parameters.

$$
\beta=C
$$

within $0.4<\lambda<0.6$ Angstroms where $\mathrm{c}$ is the fitted parameter.

$$
R=\exp \left(-E / E_{0}\right)
$$

where $E_{0}$ is the fitted parameter. Figures $36,37,43$ and 39 show the fitted parameters for energies between $0.01 \mathrm{meV}$ and $100 \mathrm{eV}$. These parameters were then fit with the magenta fit line to provide a set of six parameters as feedback for the Dakota optimization scheme. Figure 40 shows both the fitted $\mathrm{R}$ and $\beta$ parameters plotted on the same plot. By plotting $\mathrm{R}$ and $\beta$ on the same plot, it shows how both parameters take drastic turn within approximately the same energy range. This suggests that there is another energy dependence in the fitting equations for $\mathrm{R}$ and $\beta$. The dependence has not yet been formulated into the current fitting equations but is subject for future work. 


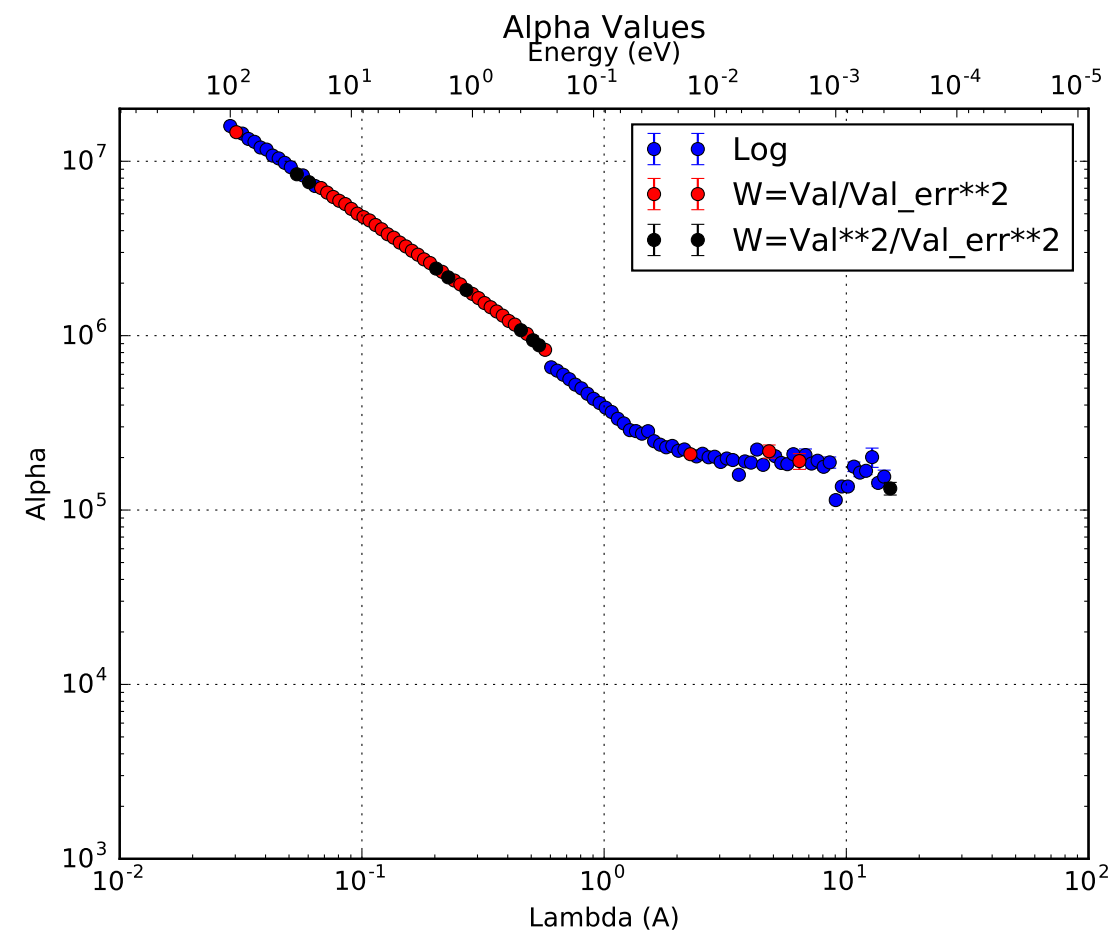

Figure 36. Fitted $\alpha$ parameters

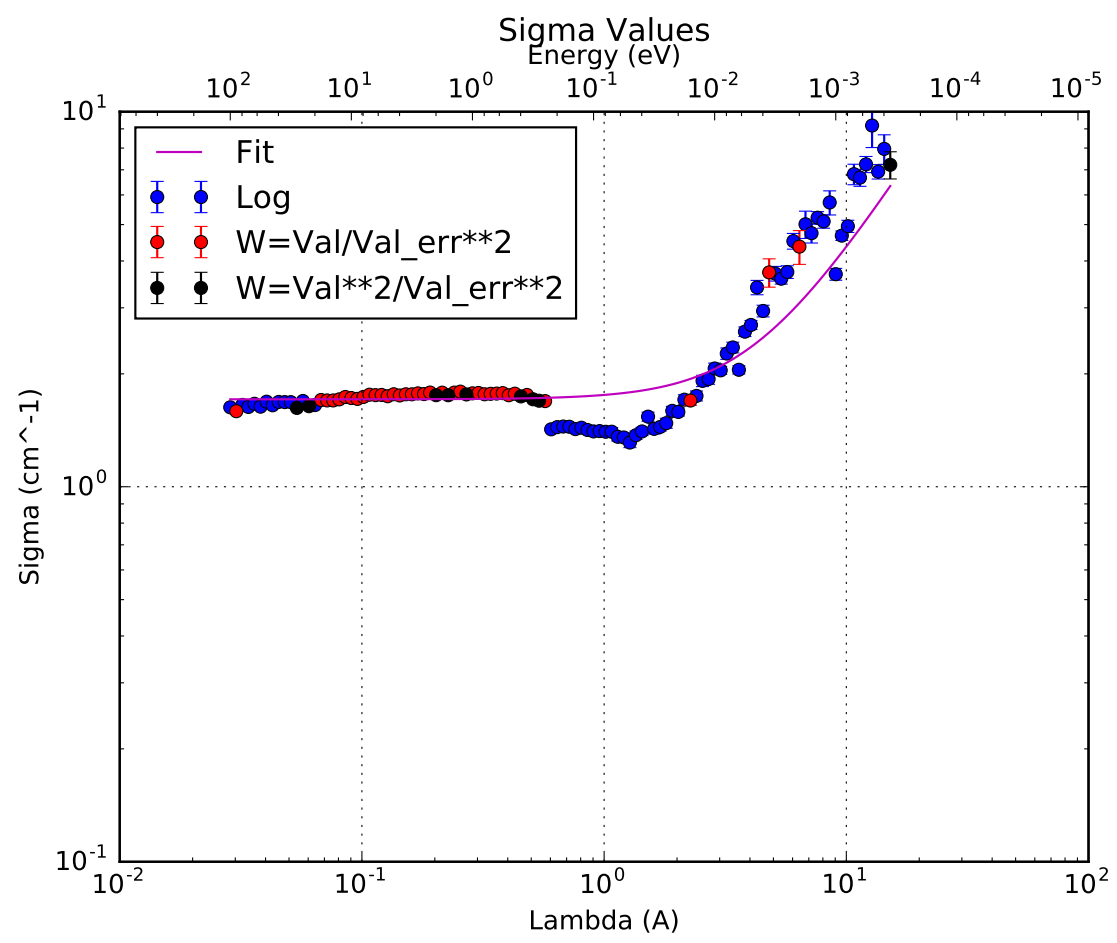

Figure 37. Fitted $\Sigma$ parameters 


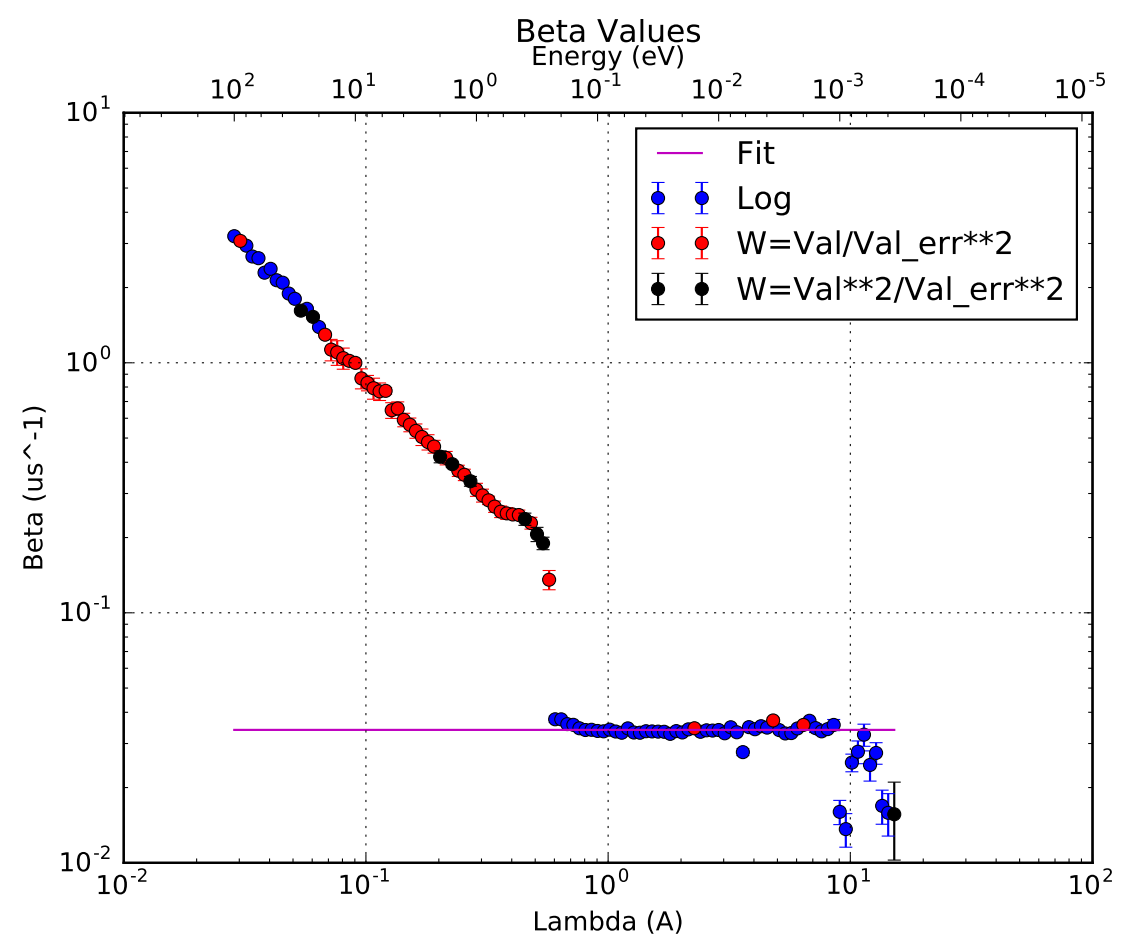

Figure 38. Fitted $\beta$ parameters

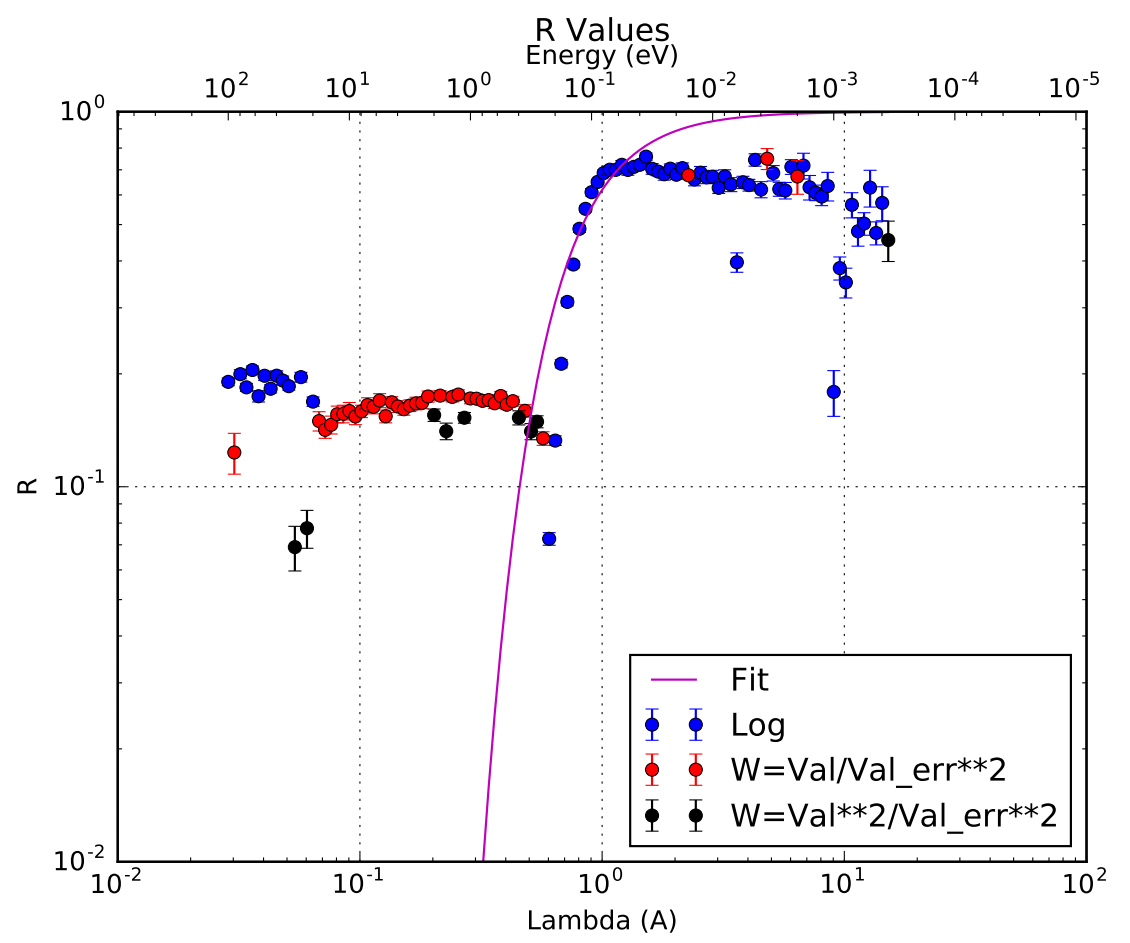

Figure 39. Fitted R parameters 


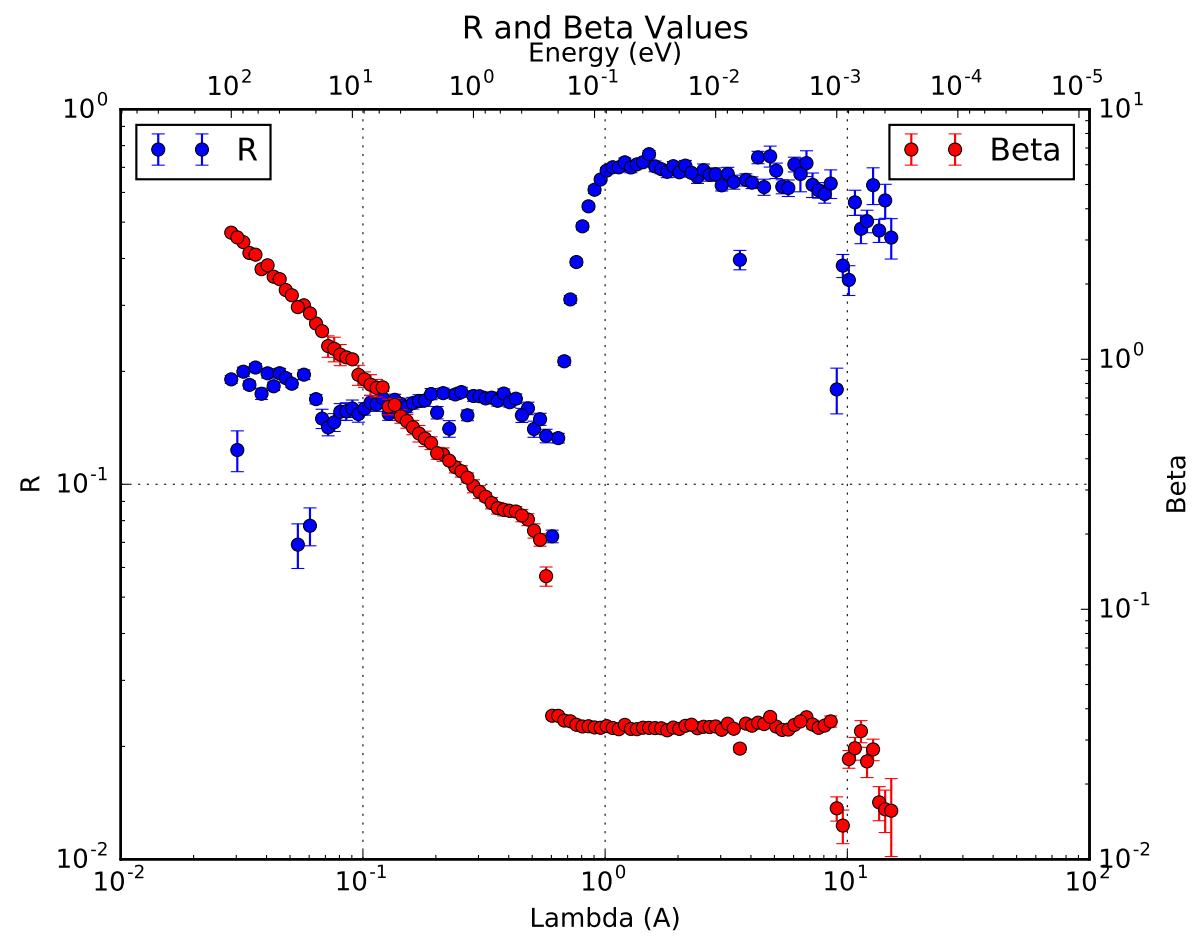

Figure 40. Fitted $\mathbf{R}$ and $\beta$ parameters 
The single set of four parameters, $S_{1}, S_{2}$, c and $E_{0}$ are then used as the parameters in the Dakota run for determining if the change in the diffusion material affected the moderator characteristic pulse shape.

However, during the testing phase of the Dakota script, these parameters were consistently off from what would be expected in a well controlled, perfectly decoupled, room-temperature water moderator. The next step was to debug what was happening inside the fitting routine to produce these incorrect characterization parameters. After color-coding the data points in Figures 36, 37, 43 and 39 by which least-squares weight was used to determine the parameter, some clear trends appeared. For the very high energies, from 15 to $100 \mathrm{eV}$, Fitting Strategy 3 was chosen, from $80 \mathrm{meV}$ to $15 \mathrm{eV}$ Fitting Strategy 1 was chosen, and from 80 $\mathrm{meV}$ and down Fitting Strategy 3 was chosen for a majority of the energies. From these plots, it was clear that the fitting routine was choosing the "best" fitting routine based upon the chi-squared value and not a bug in the code. It was then determined that the characteristic fitting equations were only valid in the energy (or wavelength) range originally used in the referenced paper, 0-6 Angstroms [5].

Figures 41, 42, 43, and 44 show the results from the Dakota parameter study routine using the fit of the IC function to characterize the neutron emission time distribution. Before performing the parameter study, the expectation was that each of the parameters should remain roughly constant over all of the configurations. The same room temperature decoupled water moderator was used in each of the configurations and neither the thickness or composition of the diffusion material should drastically change the character of the moderator. For the majority of configurations, the fitted parameters conform to the previous notion of constantcy. However, not all of the configurations met this notion and because of the nonconformity, we had to use other metrics to determine whether there is a significant change in the neutron emission time distribution (as discussed above in Section 4.3.2). At higher energies, the IC function still fits the data well, but the parameters take on new meanings. For example, the $\beta$ parameter is used to make a good fit but no longer characterizes the exponential decay. 


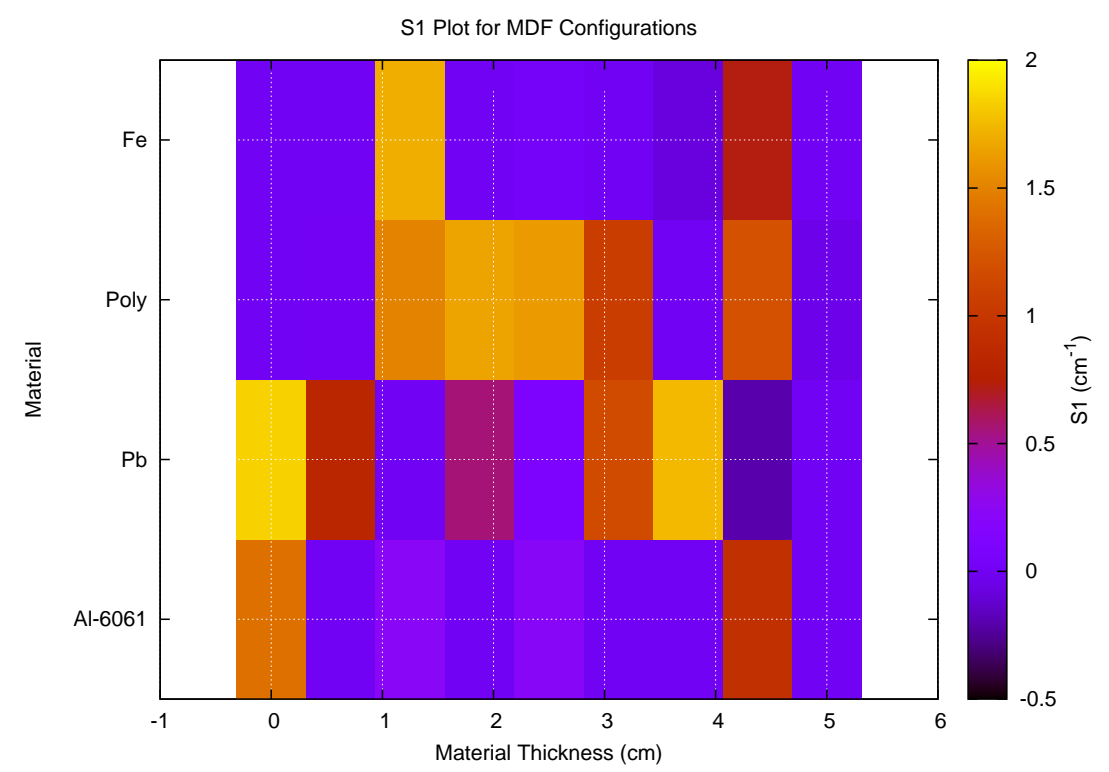

Figure 41. $S_{1}$ values from the $\alpha$ parameter fit

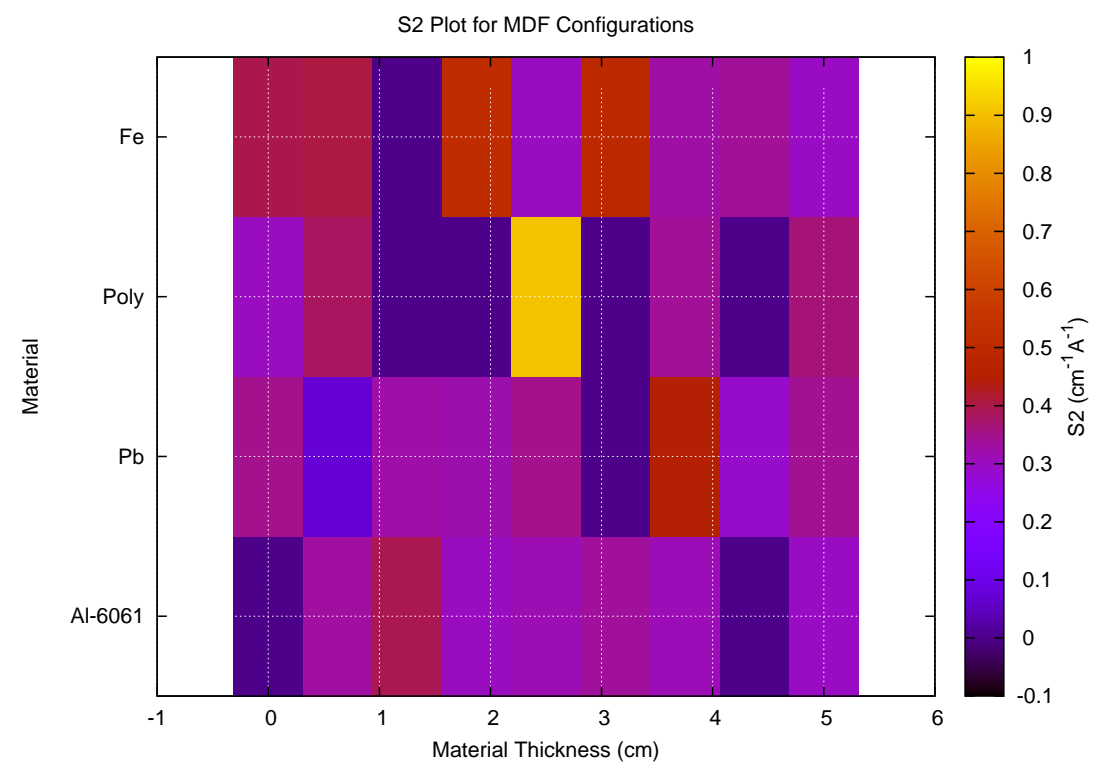

Figure 42. $S_{2}$ values from the $\alpha$ parameter fit 


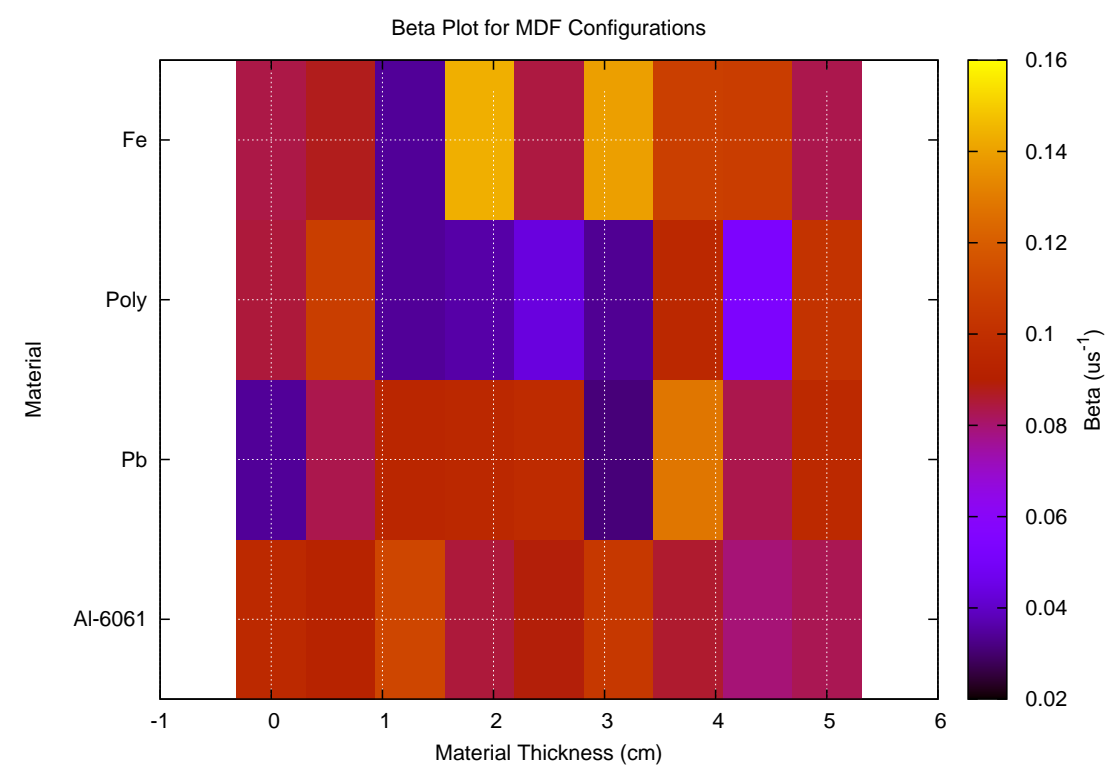

Figure 43. $\beta$ values from the $\beta$ paremeter fit

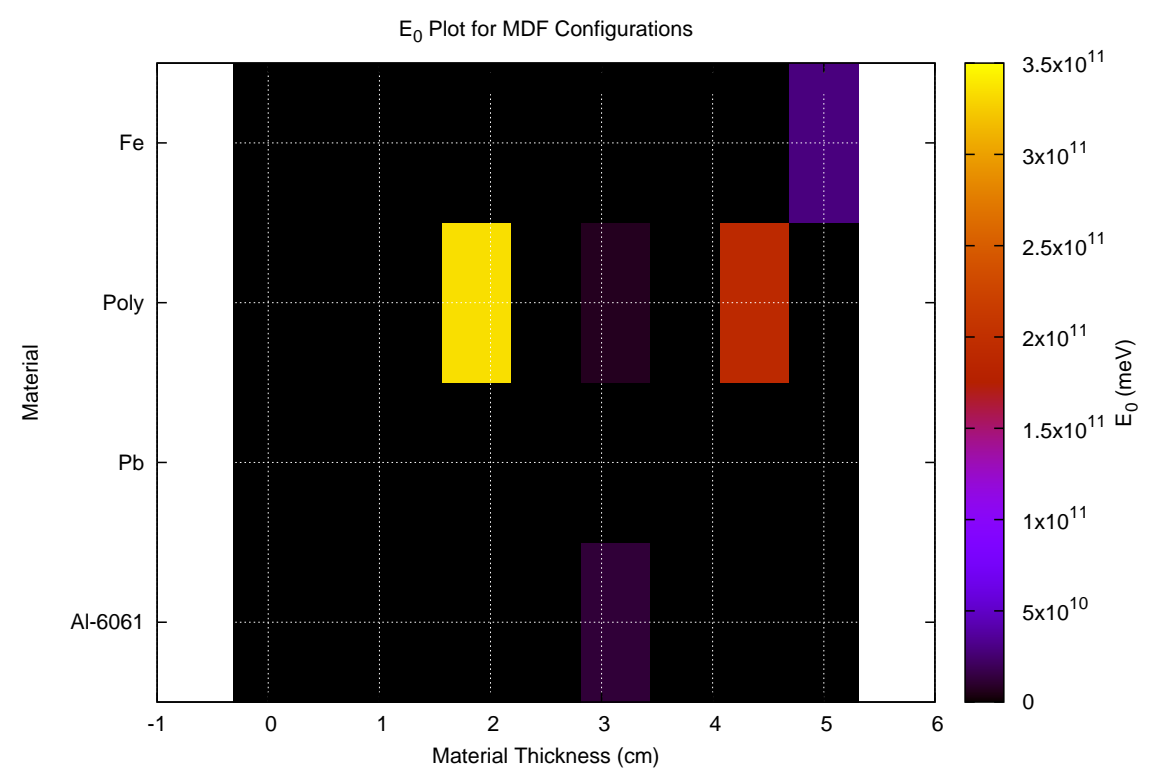

Figure 44. $E_{0}$ values from the $R$ parameter fit 
APPENDIX B. Details of Fitting the Beam Intensity 


\section{APPENDIX B. Details of Fitting the Spectral Beam Intensity}

\section{Fitting the Spectral Beam Intensity Function}

The spectral beam current function describes the spectral intensity as a function of energy of the neutrons emitted from the surface of the moderator. Fitting both the spectral beam current function and the IC function allow the full characterization of the neutrons emitting from the moderator. Equation 7 shows the spectral beam current function from the referenced paper:

$$
i(E)=i_{T h} \frac{E}{E_{T}^{2}} e^{-E / E_{T}}+i_{e p i} \frac{\Delta(E)}{\Delta\left(E_{0}\right)} \frac{1}{E}\left(\frac{E}{E_{0}}\right)^{\alpha}
$$

where $i_{T h}$ is the time-average integrated Maxwellian spectral beam current, $E_{T}$ is the mean energy of the Maxwellian, $i_{e p i}$ is the time-average epithermal spectral beam current, $\Delta(E)=\left(1+e^{\alpha \lambda-b}\right)^{-1}$, the "joining function" where $\alpha$ is the leakage exponent, and $E_{0}$ is the reference energy for the epithermal beam current [5]. Several papers show that an additional term must be included with Equation 7 in order to adequately fit the spectral beam current from a pulsed-source moderator [25, 26, 27]. Therefore, an exponential term is added to Equation 7 and variables are rearranged so that the covariance between fitted parameters is minimized. Equation 8 shows the resulting equation that is used to fit the spectral beam current during the Dakota optimization.

$$
i(E)=i_{e p i} e^{-c / \sqrt{(E)}}\left[R \frac{E}{E_{T}^{2}} e^{E / E_{T}}+\frac{1+e^{b\left(R_{a b} 0.286 / \sqrt{E_{0}}-1\right)}}{1+e^{b\left(R_{a b} 0.286 / \sqrt{E}-1\right)}} \frac{1}{E}\left(\frac{E}{E_{0}}\right)^{\alpha}\right]
$$

The following is the set of parameters used in the fitting of the spectral beam current during the Dakota optimization [5]:

- R - Ratio of $\frac{i_{T h}}{i_{e p i}}$ where $i_{T h}$ is the time-average integrated Maxwellian spectral beam current

- $i_{e p i}$ - is the time-average integrated epithermal portion of the spectral beam current

- $E_{T}$ - is the mean energy of the Maxwellian

- $\alpha$ - is the leakage coefficient

- $R_{a b}$ - is the ratio of $\frac{a}{b}$ that are used in the "joining function"

- $c$ - is the constant in the $e^{-c / \sqrt{(E)}}$ term where the term resembles the behavior of a $1 / \mathrm{v}$ absorber [26]

- $b$ - another term used in the "joining function"

Figure 45 shows the results from fitting the MDF simulated spectral beam current for a decoupled room-temperature water moderator. The strategy used to fit the spectral beam current is similar to the strategy used to fit the IC function. The fit for the spectral beam current was calculated using the same LMFIT Python package by fitting the logarithm of the data. Like the fitting strategy for the IC function, the spectral beam current is fit with rounds of fitting where the first round fits $\alpha$ and $i_{e p i}$ and then fits the rest of the parameters on the second round while holding $\alpha$ and $i_{e p i}$ constant. 


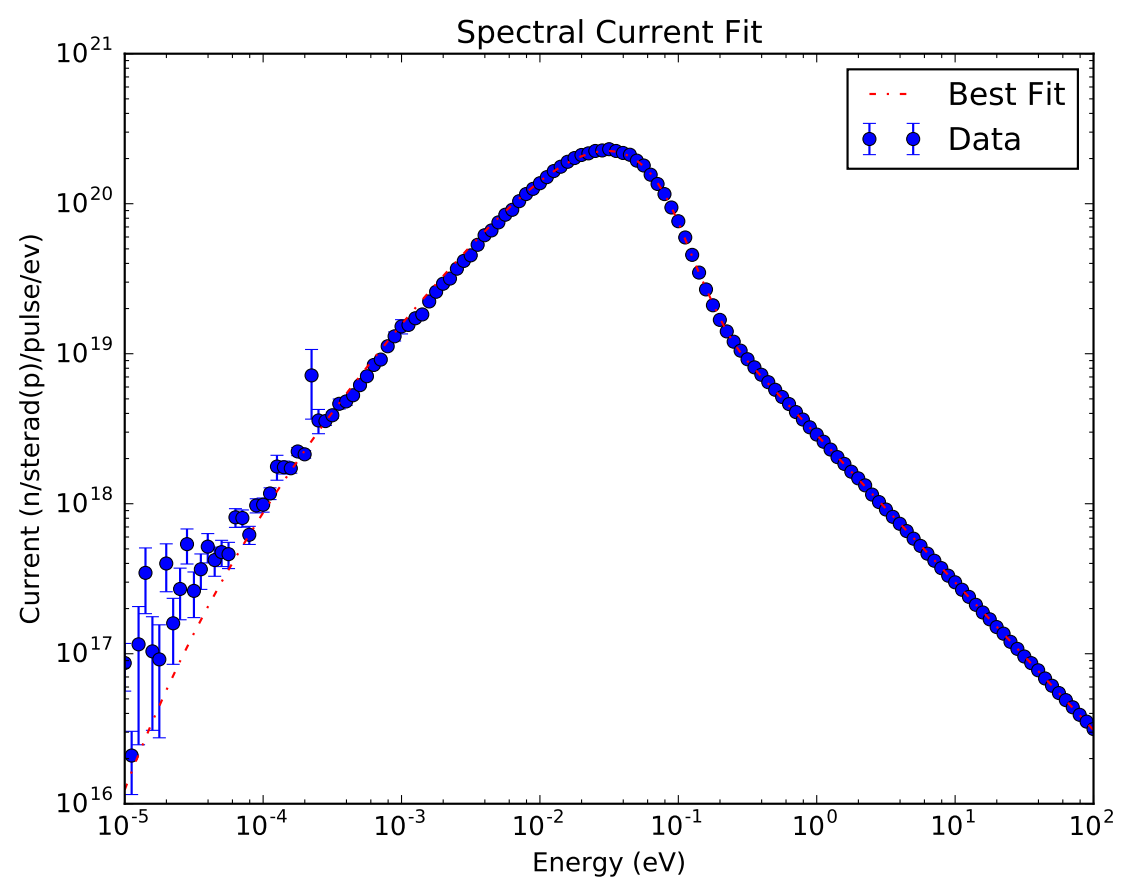

Figure 45. Fitted and Simulated MDF Spectral Intensity

Figures 46, 47, and 48 show the results from the Dakota parameter study using the fitting of the spectral beam current to determine if there was an effect from the diffusion material on the spectral beam current. The expectation for the ratio of $i_{T h} / i_{e p i}$ was that there would be a clear trend as the thickness of the diffusion material increased. However, Figure 46 shows a sporadic distribution of the ratio. Figure 47 shows $E_{T}$ values on the order of $1 \mathrm{E} 6 \mathrm{GeV}$ and that is just simply impossible. These results cannot be trusted and that is another reason why we chose to go with the methods described in Section 4.3.2. Figure 48 shows the peak values of the spectral beam current for each of the configurations and involves no fitting to determine this value. 


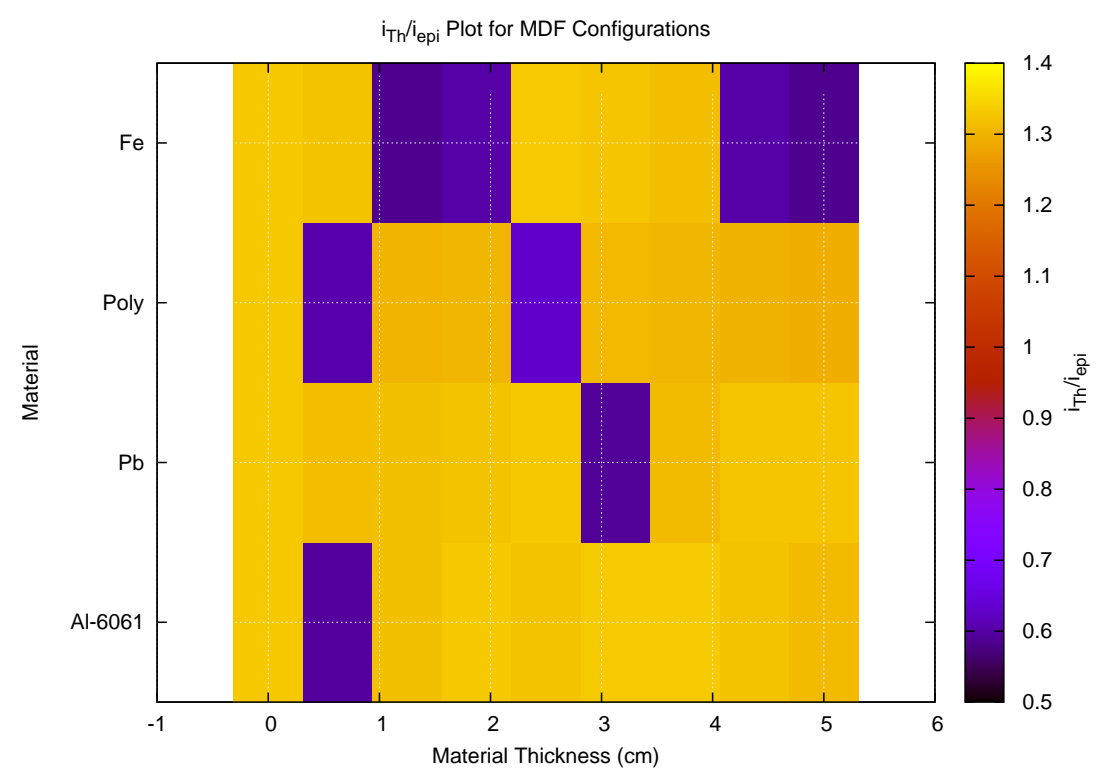

Figure 46. $i_{T h} / i_{e p i}$ values from the spectral beam current parameter fit

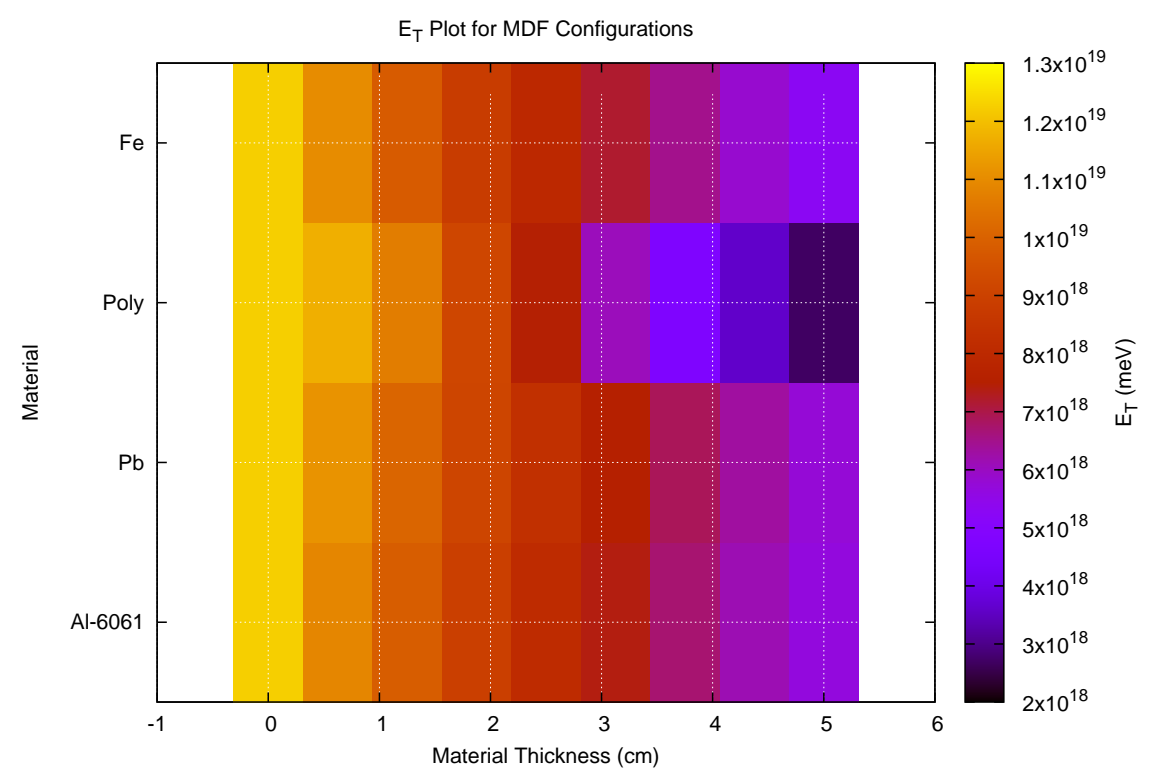

Figure 47. $E_{T}$ values from the spectral beam current parameter fit 


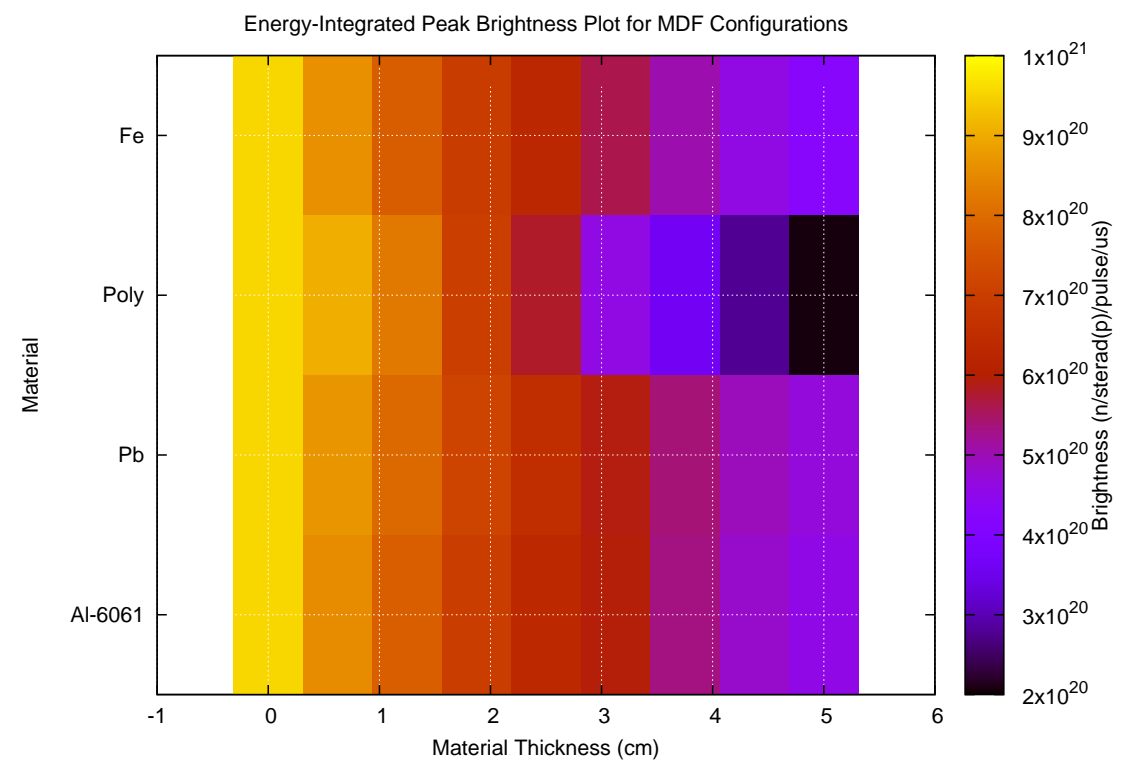

Figure 48. Energy-Integrated Peak Brightness 